\title{
Independent verification of specification models for large software systems at the early phases of development lifecycle
}

\author{
Khalid Lateef \\ West Virginia University
}

Follow this and additional works at: https://researchrepository.wvu.edu/etd

\section{Recommended Citation}

Lateef, Khalid, "Independent verification of specification models for large software systems at the early phases of development lifecycle" (1999). Graduate Theses, Dissertations, and Problem Reports. 3154. https://researchrepository.wvu.edu/etd/3154

This Dissertation is protected by copyright and/or related rights. It has been brought to you by the The Research Repository @ WVU with permission from the rights-holder(s). You are free to use this Dissertation in any way that is permitted by the copyright and related rights legislation that applies to your use. For other uses you must obtain permission from the rights-holder(s) directly, unless additional rights are indicated by a Creative Commons license in the record and/ or on the work itself. This Dissertation has been accepted for inclusion in WVU Graduate Theses, Dissertations, and Problem Reports collection by an authorized administrator of The Research Repository @ WVU. For more information, please contact researchrepository@mail.wvu.edu. 


\title{
Independent Verification of Specification Models \\ for Large Software Systems at the Early Phases of Development Lifecycle
}

\section{Khalid Lateef}

\author{
Dissertation Submitted to the \\ College of Engineering and Mineral Resources at \\ West Virginia University \\ in partial fulfillment of the requirements for the \\ Doctor of Philosophy in Computer Engineering
}

\author{
H. Ammar, Ph.D., Chair \\ M.A. Choudhry, Ph.D. \\ S. Easterbrook, Ph.D. \\ P. Klinkhachorn, Ph.D. \\ J. Mooney, Ph.D.
}
Department of Computer Science \& Electrical Engineering West Virginia University Morgantown, WV 26506

1999

Keywords: Software system models, dynamic analysis, SART, UML, Independent Verification and Validation Copyright 1999 Khalid Lateef 


\section{Acknowledgment}

To my parents Razia and Abdul Lateef, whose teachings of being patient and diligent were utmost important in helping me to finish this work. I am ever thankful to my wife Atiya and daughters Sundus and Soofia for their emotional support. They gave up a lot of their precious family time with me, so that I can finish my research.

From the very beginning, my advisor Dr. H. Ammar was instrumental in helping me to focus on this research topic. I am indebted to him for his guidance, patience and continuous mentoring that brought me to this stage. I am thankful to my collegues at graduate school, Vinay Mogolothu and Tooraj Nikzadeh for their help in building models and providing simulation results.

I really appreciate the efforts of my committee members Dr. Choudhry, Dr. Easterbrook, Dr. Klinkhachorn and Dr. Mooney for reviewing the draft of my dissertation. Special thanks are due to Dr. Easterbrook for his help in my work related to the topics of metamodeling and object oriented notations. I am also thankful to the following professors for their support and guidance: Dr. Noore, Dr. Klien, Dr. Nutter, and Dr. Callahan.

Special thanks are due to George Jackelen, whose proof reading and advice on the style and write up of this work was of immense help. I will be forever thankful to our NASA representative John Hinkle, and my colleagues at NASA IV\&V facility in Fairmont, specially Tim Davis, Van Casdorph, Jill Broadwater, Scott Radabaugh, and Jason Cooper, Larry Ullom, David Knepper, Doug Starfield, Jim Dalton, and Mary Robinette.

Finally, I would like to thank my seniors at Averstar Inc. who remained supportive throughout the process of writing this dissertation: Sarma Susarla, Greg Miller, Dan McCaugherty, and John Dicks . 


\title{
ABSTRACT
}

\author{
INDEPENDENT VERIFICATION OF SPECIFICATION MODELS FOR \\ LARGE SOFTWARE SYSTEMS \\ AT THE EARLY PHASES OF SOFTWARE DEVELOPMENT LIFECYCLE
}

\author{
by Khalid Lateef \\ Advisor: Dr. Hany H. Ammar
}

One of the major challenges facing the software industry, in general and IV\&V (Independent Verification and Validation) analysts in particular, is to find ways for analyzing dynamic behavior of requirement specifications of large software systems early in the development lifecycle. Such analysis can significantly improve the performance and reliability of the developed systems. This dissertation addresses the problem of developing an IV\&V framework for extracting semantics of dynamic behavior from requirement specifications based on:

1. SART (Structured Analysis with Realtime) models, and

2. UML (Unified Modeling Language) models.

For SART, the framework presented here shows a direct mapping from SART specification models to CPN (Colored Petrinets) models. The semantics of the SART hierarchy at the individual levels are preserved in the mapping. This makes it easy for the analyst to perform the analysis and trace back to the corresponding SART model. CPN was selected because it supports rigorous dynamic analysis. A large scale case study based on a component of NASA EOS system was performed for a proof of the concept.

For UML specifications, an approach based on metamodels is presented. A special type of metamodel, called dynamic metamodel (DMM), is introduced. This approach holds several advantages over the direct mapping of UML to CPN. The mapping rules for generating DMM are not CPN specific, hence they would not change if a language other than CPN is used. Also it makes it more flexible to develop DMM because other types of models can be added to the existing UML models. A simple example of a pacemaker is used to illustrate the concepts of DMM. 


\section{Table of Contents}

Chapter 1 Introduction and Background $\ldots \ldots \ldots \ldots \ldots \ldots \ldots \ldots \ldots \ldots$

$1.1 \quad$ Independent Verification and Validation $(\mathrm{IV} \& \mathrm{~V}) \ldots \ldots \ldots \ldots \ldots \ldots 2$

$1.2 \quad$ Importance of IV\&V at early phases of development lifecycle . . . . . . . 5

$1.3 \quad$ IV\&V using simulation of dynamic models $\ldots \ldots \ldots \ldots \ldots \ldots \ldots$

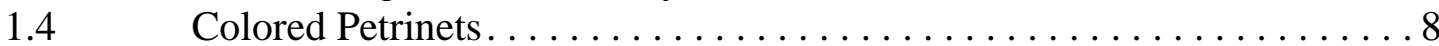

1.5 SART notation for generating specifications $\ldots \ldots \ldots \ldots \ldots \ldots \ldots$

1.5.1 Data Flow and Control Flow Diagrams (DFDs/CFDs) . . . . . . . 15

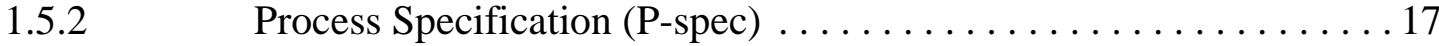

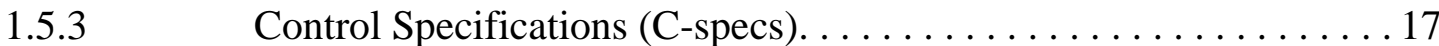

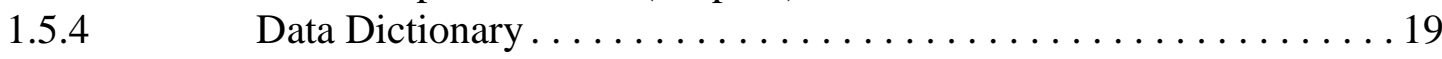

$1.6 \quad$ UML notation for generating specifications . . . . . . . . . . . . 19

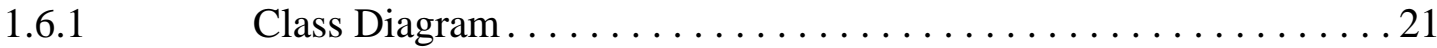

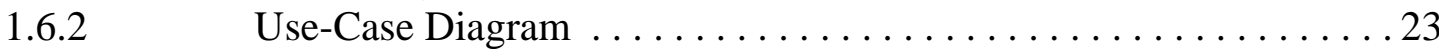

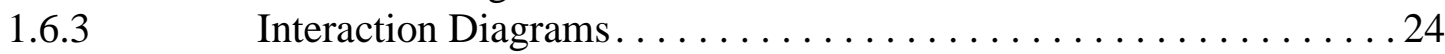

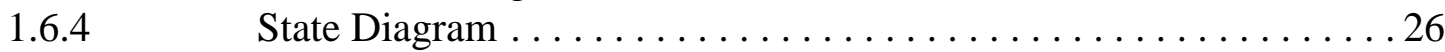

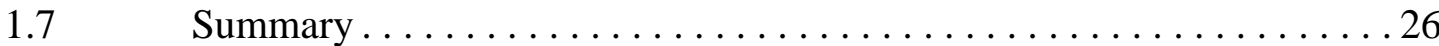

Chapter 2 Problem Statement and Objectives $\ldots \ldots \ldots \ldots \ldots \ldots \ldots \ldots$

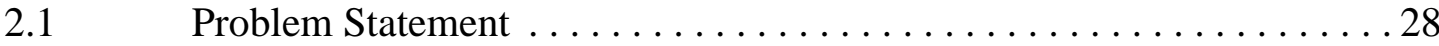

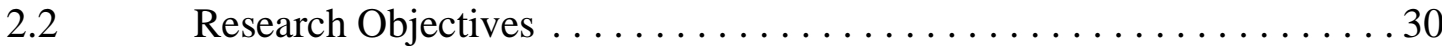

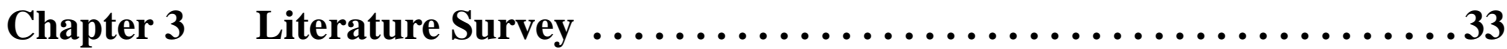

$3.1 \quad$ Formal specifications of software systems $\ldots \ldots \ldots \ldots \ldots \ldots \ldots \ldots$

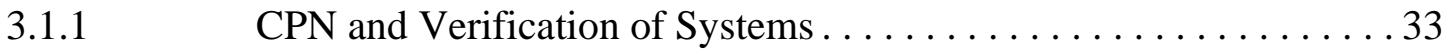

3.1.2 Graph Grammar for Extracting Syntax and Semantics.......... 35

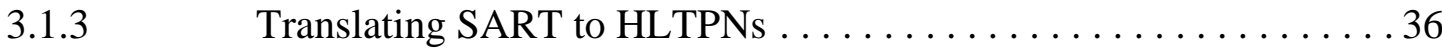

3.1.4 Translating SART to Calculus of Communicating Systems (CCS) . 36

3.1.5 Formalizing and Integrating the Dynamic Model within OMT . . . . 37

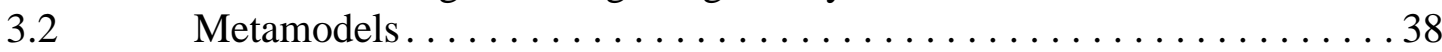

3.2.1 Using Metamodels to address multiple requirements perspective . . . 39

3.2.2 Problems in Metamodeling . . . . . . . . . . . . . . . . . 39

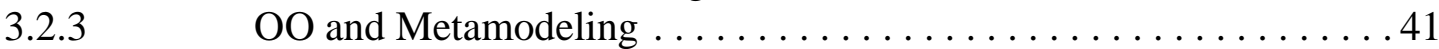

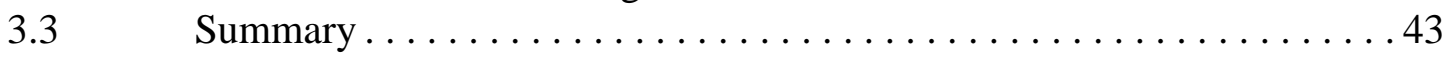

Chapter 4 Dynamic Analysis of SART Models................. 44

4.1 Comparison of the SART and the CPN Environments. . . . . . . . 46

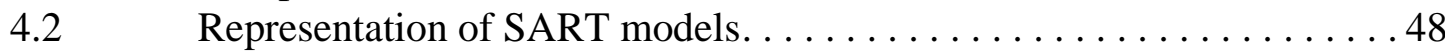

$4.3 \quad$ Representation of CPN models . . . . . . . . . . . . . . . . . 49

$4.4 \quad$ Mapping the SART model to a HCPN model. . . . . . . . . . . . . 50

4.5 Mapping the Data Flow Diagram to CPN page . . . . . . . . . . . . . 50

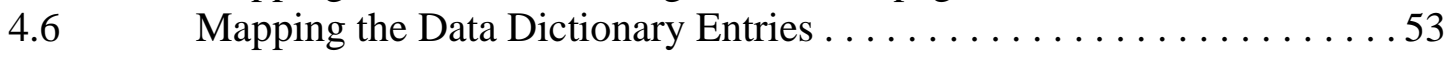

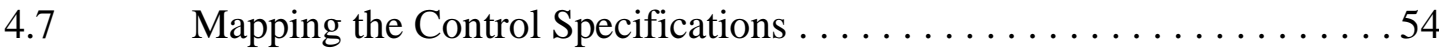




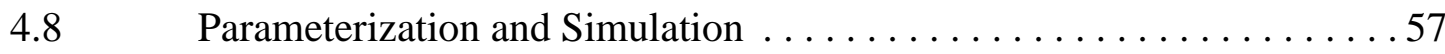

4.9 Tracking upgrades in Developer's models . . . . . . . . . . . . . 58

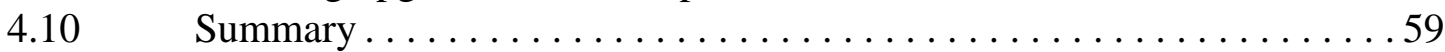

Chapter 5 A Framework for Dynamic Analysis of UML . . . . . . . . . . 60

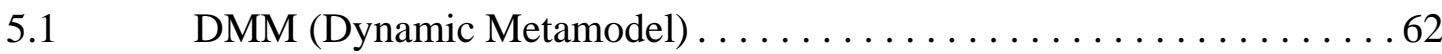

5.1.1 Metamodels as Applied to Software Development. . . . . . . . . 63

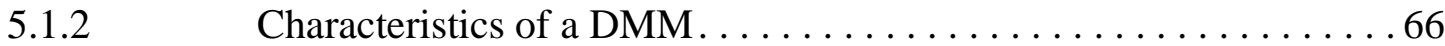

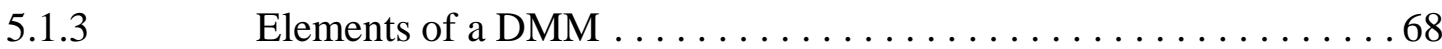

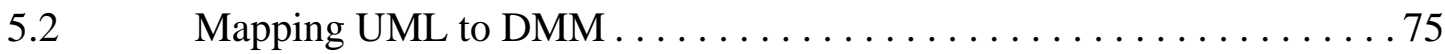

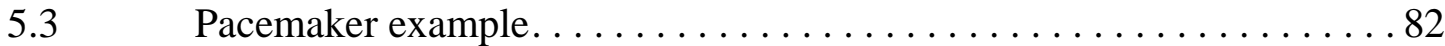

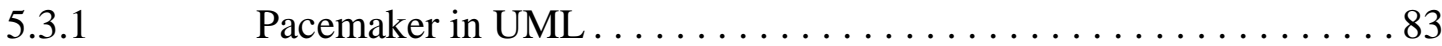

5.3.2 DMM model . . . . . . . . . . . . . . . . . . . . 89

$5.4 \quad$ Generating CPN models from DMM $\ldots \ldots \ldots \ldots \ldots \ldots$

Chapter 6 Application of Methodology

to a Large Software System . . . . . . . . . . . . . . . . . 94

$6.1 \quad$ Description of the Commanding subsystem of EOS . . . . . . . . . 94

$6.1 .1 \quad$ Build_Spacecraft_Realtime_Command $\ldots \ldots \ldots \ldots \ldots \ldots \ldots \ldots 9$

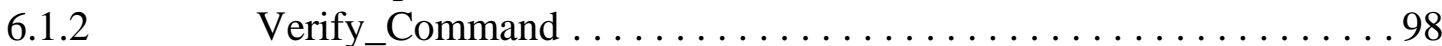

6.1.3 Evaluate_Spacecraft_Command_Status ...................... 98

6.2 Dynamic Modeling of the Commanding subsystem . . . . . . . . . . 102

6.3 Dynamic Analysis of the Commanding Component .............. 114

6.4 Performance Analysis of the Commanding Component. . . . . . . . . . . 115

6.4.1 Performance under normal sequential execution . . . . . . . . . 116

6.4.2 Performance under pipelined normal execution . . . . . . . . . 116

6.4.3 Performability analysis of the pipelined design . . . . . . . . . 121

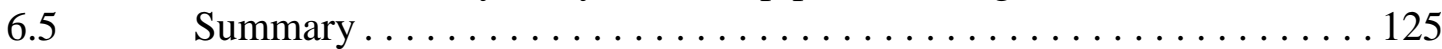

Chapter 7 Summary, Conclusions, and Future Work ............. 127

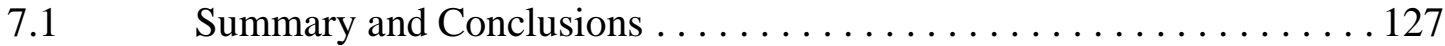

$7.2 \quad$ Future work . . . . . . . . . . . . . . . . . . . . . . . 129

7.2.1 Tool support for generating dynamic models . . . . . . . . . . . 129

7.2.2 Explore new dynamic specification metrics. . . . . . . . . . 131

7.2.3 Methods for improving the design process of operational profiles. . 131

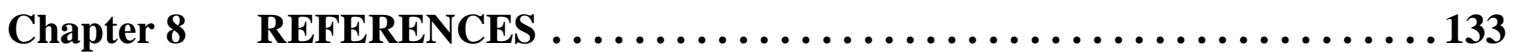




\section{Chapter 1 Introduction and Background}

This work addresses an important aspect of large software system development: how to verify the dynamic behavior of software systems early in the development lifecycle. The focus is on the process and tasks to be followed by an analyst who may not be a part of the software development team. An example of such analysis is the one performed by IV\&V (Independent Verification and Validation) analysts. IV\&V and its link with the processes of software engineering are described later sections of this chapter.

This dissertation presents frameworks for generating dynamic models from requirement analysis models. These frameworks contribute to two areas of the state of art in software engineering. These areas of contributions are based on the following four stages of the proposed process:

- The semantics are extracted from given software specifications.

- The semantics of the extracted specifications are mapped to formal dynamic models.

- The dynamic models are utilized to build scenario based simulation models.

- The simulation models are used to perform dynamic analysis of the software system.

The frameworks based on these stages are scalable and can also be automated. The mapping stage preserves the level of abstraction and granularity in the original software specifications. The resulting dynamic models are scalable. This is different from the approaches discussed in the literature (Chapter 3). Automation makes the framework presented in this work readily applicable to large scale software systems. An example describing these stages for a large software system is described in Chapter 6. 
The two areas of contributions are:

- SART (Structured Analysis with Real Time): The first area of contributions is based on the artifacts generated in the four stages when SART is used as input to the dynamic analysis. These artifacts are modular and provide better representation as compared to the techniques used in earlier work. This again makes the proposed technique more suitable for large scale systems. Chapter 4 contains more details of this area of contributions.

- UML (Unified Modeling Language): The second area of contributions deals with generating dynamic models for UML. To facilitate this activity, a new class of dynamic models, termed dynamic metamodels (DMM) is introduced. Metamodels in general may cover different aspects such as graphical representation of elements, tool implementation, and development of overall semantics for a modeling notation. DMM on the other hand are specific for representing dynamic behavior only. Chapter 5 gives more details of this area contributions.

The analysis techniques, as introduced in the preceding paragraphs, are especially suitable for IV\&V of large software systems by providing means to analyze software systems early in a development lifecycle. The following sections contain a brief introduction to IV\&V and its role in software development. The definition of IV\&V is given in the Section 1.1. Section 1.2 describes the importance of software systems' IV\&V during the early phases of development lifecycle. Section 1.3 explains the role of the dynamic simulations of software system models at the early phases of a software lifecycle.

\subsection{Independent Verification and Validation (IV\&V)}

The process and product of software development can greatly benefit from the use of IV\&V. It will be prudent to give a brief description of software development phases before 
defining the concepts of IV\&V.

The lifecycle phases for a sample software development process are listed in Table-1. The first column lists the phases, and the second column assigns them a serial number $\mathrm{N}$. Development of software systems starts with requirements definition. Once defined, the requirements are analyzed to remove ambiguities and inconsistencies. The top-level design phase starts when the requirements (or some portion there of) are mature enough. This phase is followed by detailed design and implementation. Implemented software goes through integration and testing in the final phase of the lifecycle.

\begin{tabular}{|c|c|}
\hline Lifecycle Development Phases & Serial Number (N) \\
\hline \hline Software requirements definition and analysis phase & 1 \\
\hline Software top-level design phase & 2 \\
\hline Software detailed design and implementation phase & 3 \\
\hline Software integration and testing phase & 4 \\
\hline
\end{tabular}

Table 1-1 Lifecycle phases

Verification, as perceived in $I V \& V$, is the independent evaluation process designed to ensure the consistency and completeness of the software product at any given phase within the development lifecycle. Consistency is concerned with measuring the degree a given phase "N" (Table 1-1) is in agreement with the previous phase, i.e., "N-1". Completeness is a measure of the readiness of phase $\mathrm{N}$ to initiate the next phase, i.e., $\mathrm{N}+1$, in the lifecycle. Validation deals with software test and evaluation to measure how well the software executes according to established requirements [2].

There are two methods of $\mathrm{V} \& \mathrm{~V}$ (Verification and Validation) being used in the process of software development. The first is the traditional method and the second is the IV\&V based method (Figure 1-1). Both of these methods involve a customer and a developer. The customer is 
an entity putting out the requirements for a new software system. The developer is the entity responsible for building the required software system based on these requirements. These two entities could be part of the same organization. The developer could have an internal V\&V team (Figure 1-1). In this situation the customer may not have access to the product evaluation reports generated by the internal V\&V team.
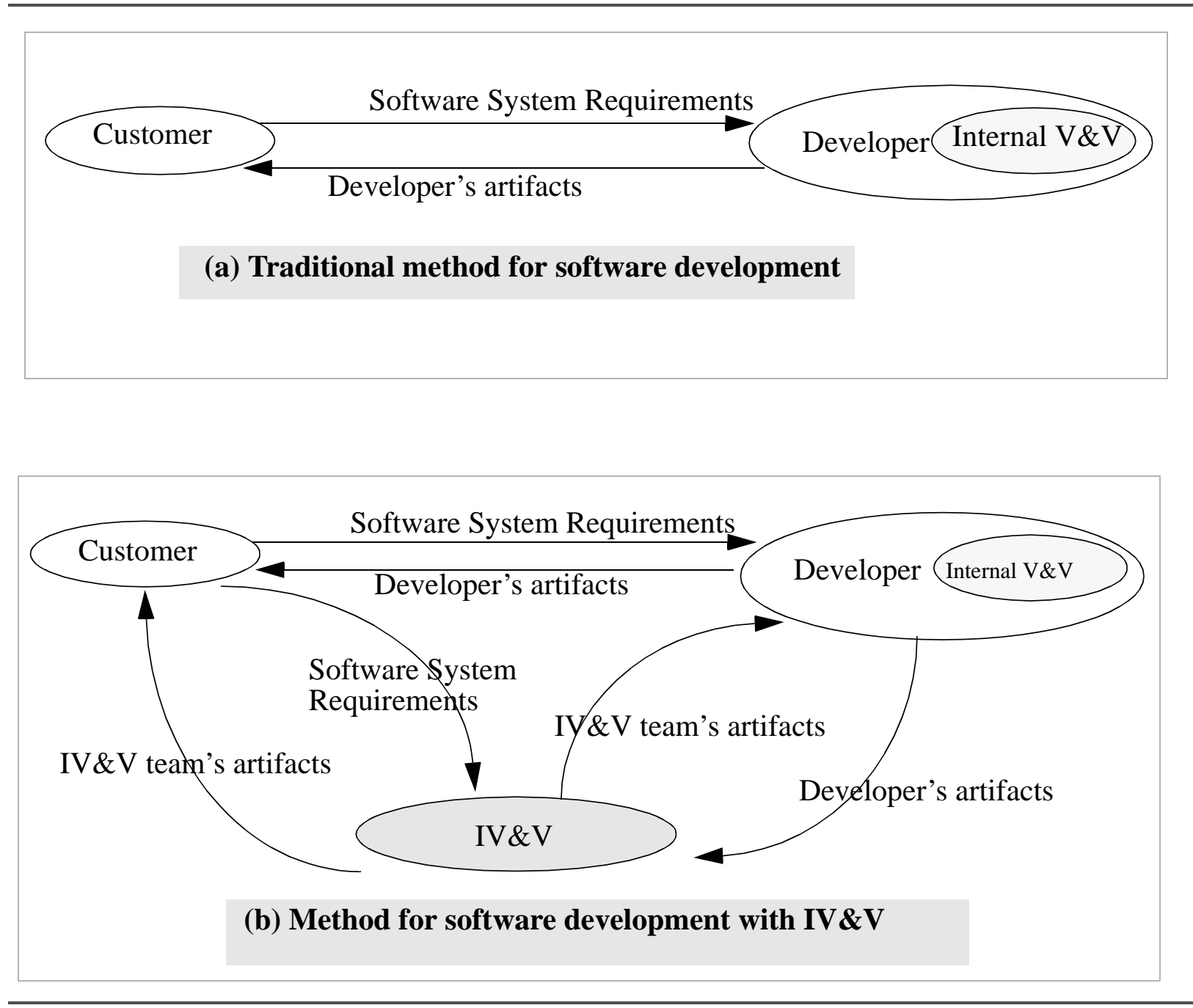

Figure 1-1 Comparison of software development methods

The customer sends the set of requirements to the developer (Figure 1-1). These requirements are generally very high level and may contain a brief description of the needed 
software system. The developer utilizes these requirements to generate software system artifacts, consisting of the developed software system along with a set of documents. These documents may include software requirement specifications, top level design documents, software test description documents, interface control documents, data dictionary documents, user manuals, etc. Documents change as the system evolves through different phases of development lifecycle.

Figure 1-1.a shows the traditional arrangement of software system development. Two entities, i.e., customer and developer interact with each other, with their specific roles as explained above. Figure 1-1.b shows a different kind of setup where a third entity (IV\&V team) takes an active role. The IV\&V team analyzes the requirements from the customer as well as the artifacts generated by the developer. Once the IV\&V analyst finds an issue, IV\&V submits it to the developer and sends the appropriate feedback to the customer. The following section describes, in greater detail, the role of IV\&V during the development lifecycle.

\subsection{Importance of IV\&V at early phases of development lifecycle}

IV\&V during the early phases of software development is critical in reducing the expected number of faults in a software system. Software requirements errors have been found to account for a majority of production software failures [3]. This increases the importance of using IV\&V early in the development lifecycle. Some of the objectives of an early IV\&V effort can be summarized as follows:

- Analysis of software system that is independent of the developer team,

- Enhances ability to detect problems early in the lifecycle (a major threat to any development) by providing an ability to perform rigorous analysis of dynamic behavioral properties, i.e., "tests" early in the development,

- additional verification of requirements integrity in terms of consistency, syntactical correctness and completeness, 
- procedures for estimating performance, risk, complexity and reliability in the early phases of the development lifecycle.

The benefits of IV\&V may include:

- an appraisal of the status of software development activities independent of the developers's view,

- increased confidence the customer will obtain a quality product by identifying and resolving problems early in the lifecycle,

- Unbiased reviews, evaluations and related reports.

As shown by the work of Nancy Leveson, realtime safety critical software systems may suffer from poor quality, under-estimated maintenance effort and unexpected operational aspects. This is supported by her work on accidents related to Therac -25 [4]. A well-qualified IV\&V team can ensure significant cost savings and improved quality of software systems. A key attribute of a successful IV\&V team is the ability to focus on inadequacies and problems in requirements with the goal of contributing to the correction of potential problem areas early in the development lifecycle. Complete and unambiguous software requirements are a necessary [3] foundation for the software development of large complex systems and the necessary standard for measuring software quality. For critical components of a software system, IV\&V activity can provide an evaluation of the requirements to measure qualities such as testability, traceability to higher-level specifications, completeness, adequacy and degree of ambiguity that may exist in the software specifications.

IV\&V technical support provides an in-depth technical visibility to the customer. This enables the customer to maintain control of the overall software. IV\&V emphasizes the importance of quality specifications, development standards and IV \&V procedures. It also helps in effective configuration management, comprehensive design reviews, quality test planning and 
documentation, and independent validation.

\subsection{IV\&V using simulation of dynamic models}

Simulation based dynamic analysis can help perform rigorous analysis of dynamic properties early in the development lifecycle. The dynamic analysis can detect problems as well as ensure consistency and completeness of the requirements. An IV\&V analyst needs executable specifications and a simulation tool to perform dynamic analysis. Executable specifications (dynamic analysis) can be developed using one of the following methods:

- from the textual requirements,

- utilize the analysis models constructed by the developer.

The first method is very time consuming and not feasible for very large software systems. As observed by Cheng et. al., [1], "attempting to construct a formal specification directly from an informal, high-level requirements document can be challenging". During the course of development, requirements for a software system may change frequently. This needs much effort by IV\&V analyst to recreate the executable specifications every time the software system requirements change. This method also poses a serious problem in terms of IV\&V models, i.e., being consistent with the developer's model. The second method can alleviate some of these consistency problems.

Developers generally use informal techniques such as SART or OO (Object-Oriented) for requirements modeling and specifications. Maier [5] has listed various advantages in using these informal methodologies. Integrated development environments (e.g., Integrated CASE (ICASE) tools) have evolved to support a number of notations for requirements modeling using SART as well as OO. Such informal specifications are scalable and are being used in large industrial projects. Being informal, these models are not executable. 
Conceptual grammars for requirement specifications are classified by Fraser and Kumar [6] into two major groups: informal specifications and formal specifications. Informal specifications models supported by CASE tools used in industry are based on SART models or Object-Oriented Analysis (OOA) models. Formal specifications are based on formal languages such as VDM, Z and Petrinets.

Informal specification languages use a combination of graphics and semiformal textual grammars to describe and specify software system requirements [6], [7], [8]. These languages are ideal for the developer's environment as they make it convenient for both user and developer to communicate with each other and refine the user description to a set of informal requirements documents. These languages tend to be imprecise and ambiguous. Hence there is clearly a need to use formal specification languages for the requirements analysts domain [9].

A formal notation can be analyzed and manipulated using mathematical operators. Mathematical proof procedures can be used to test and verify the internal consistency and syntactic correctness of the specifications [6]. Formal languages provide exactness and the ability to reason [8]. If the problem can be specified mathematically, then a program can be developed and proven to satisfy the specifications. The use of formal notation is limited to small systems due to different reasons which may include scalability problems, difficult to understand by system developers, and the lack of COTS tool support.

\subsection{Colored Petrinets}

Petrinets were invented for verification of communication protocols in 1960s [10]. A Petrinet is a particular kind of directed graph, together with an initial state called initial marking. The underlying graph of a Petrinet is a directed graph consisting of two kinds of nodes, called places and transitions, where arcs are either from a place to a transition or from a transition to a place. 
Petrinets can be used to model properties such as process synchronization, asynchronous events, concurrent operations and conflicts or resource sharing. They can also be viewed as precise and lucid specifications of the programs to be implemented. Petrinets have been used for modeling and analysis of concurrent and parallel systems, communication protocols, performance evaluation and fault-tolerant systems. Because of the uniformity of the basic net elements, they are strong design tools in modeling a variety of systems within the same development environment. For example, hardware and software systems can be modeled together. Modeling a system using Petrinets has many advantages such as: the overall behavior is easier to understand due to the graphical (Figure 1-2) and precise nature of the presentation scheme. Dynamic features of Petrinets make them suitable for modeling complex concurrent and nondeterministic processes [11]. One of the main limitation of petrinets have been the explosion of nets for even a small system.

Colored Petrinets (CPNs) extend petrinets by augmenting them with the units of information flow or tokens are given types or colors. They also provide mechanisms for setting up models in a hierarchical form. CPN models can be interactively executed and analyzed using commercial-off-the-shelf (COTS) tools like Design/CPN. The Design/CPN package is developed by Meta Software Corporation, USA, in close cooperation with researchers from the CPN group at Aarhus University, Denmark. This work also uses Design/CPN for analysis of large software systems (Chapter 4, Chapter 5 and Chapter 6). More information is available from the home page for Design/CPN at "http://www.daimi.au.dk/designCPN/".

CPNs use data types, data objects and variables. CPN data types are called colorsets and CPN data objects are called tokens. A simple CPN is composed of the following graphical elements (Figure 1-2):

- Places (represented by circles or ovals) locations for holding data.

- Transitions (represented by bars or rectangles) activities that transform data. 
- Arcs (represented by arrows) connect places with transitions to specify data flow paths.

- Arc Inscriptions: Input arc inscriptions specify the data existing for an activity to occur. Output arc inscriptions specify the data produced if an activity occurs.

- Guards define conditions that must be true for an activity to occur.

- Marking is an assignment of tokens (black dots) to the places of the net.

The definitions of the colors and declaration of CPN variables of different colors are

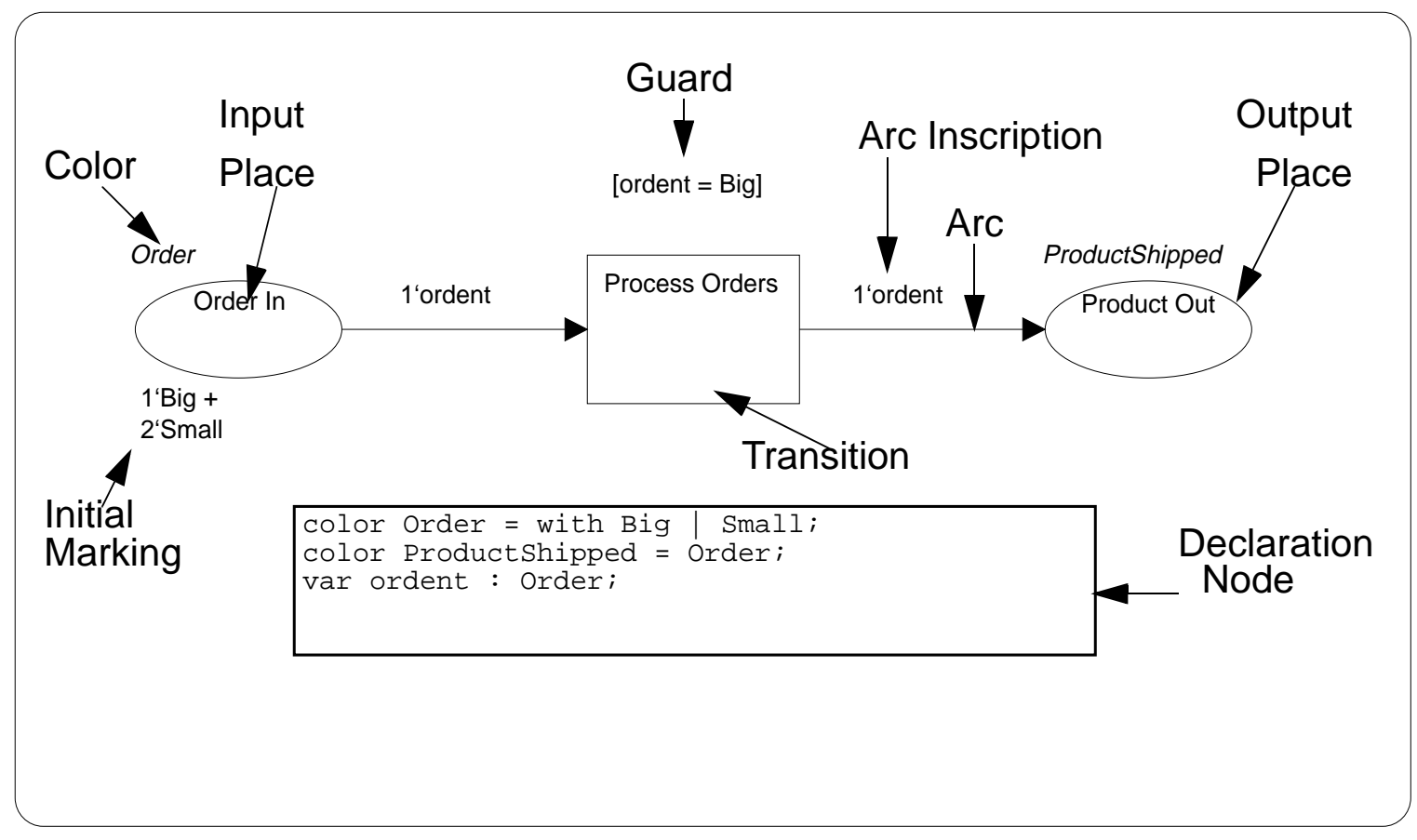

Figure 1-2 Example of a CPN

specified in a declaration node (Figure 1-2). In the declaration node, two colors are defined: Order and ProductShipped. Order can take the values "Big" or "Small". A CPN variable called ordent of color Order is also declared. An input place of color Order named "Order In" and an output place of color ProductShipped named "Product Out" are connected to the transition "Process Orders". The number of tokens and their values present in a place in the initial state of the model are represented as the initial marking of a place. In Figure 1-2, the initial marking of the place 
Order_In is specified as one Big token and two small tokens of the color Order. The arc inscription of the input arc from "Order In" place to "Process Orders" transition is specified as one token of ordent. A guard is a boolean expression associated with a transition. The guard for the transition "Process Orders" is specified as "ordent = Big". This means the transition "Process Orders" can fire only if the value of the variable ordent is one Big token of color Order. A transition is enabled if and only if each of its input places contain at least as many tokens as specified by the input arc inscriptions and if the guard evaluates to true. When a transition is enabled, it may fire, i.e., all enabling tokens are removed from the input places and tokens (according to the code written in the code segment, if any) are deposited in each of the output places. At any given time instance, the distribution of tokens on places defines the current state of the modeled system. By adding arc inscriptions to the input arcs (g) and/or by adding a guard to the transition itself $(\mathrm{G})$, one can control the enabling conditions of the transition. Transitions in a CPN could have code segments (C) where one can implement the exact transformation from input data to output data using CPN ML (Meta Language). A basic CPN comprising two places and one transition is shown in Figure 1-3. The corresponding dynamics of this CPN model could be summarized as:

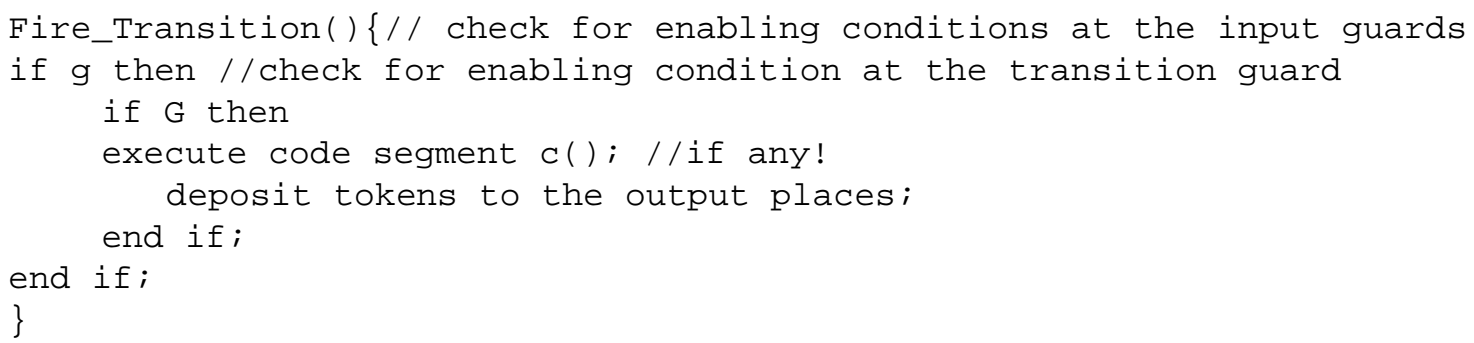




\section{Transition}

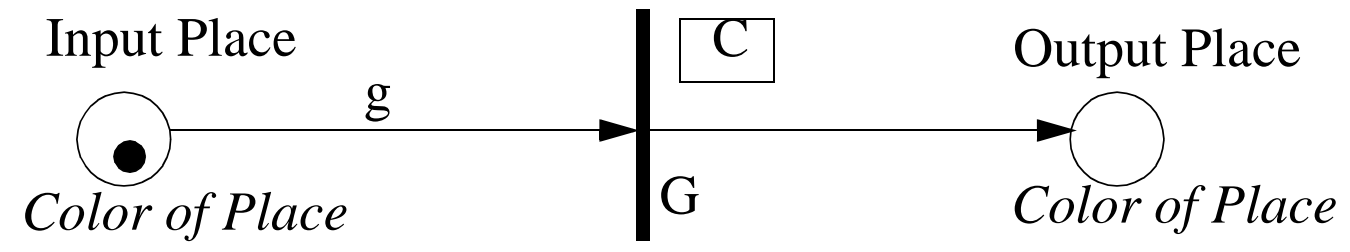

Figure 1-3 Basic CPN

The CPN Editor supports construction, modification and syntax check of CPN models. The CPN Simulator supports interactive and automatic simulation of CPN models and the Occurrence Graph Tool supports construction and analysis of occurrence graphs for CPN models. These three tools can be used for modeling and analysis of large hierarchical CPNs. The analysis can be performed with time specifications to assess the performance/performability aspects of a system.

Figure 1-4 shows the hierarchy of $\mathrm{CPN}$ as shown in the Design/CPN tool. One of the transitions of the top level CPN-PAGE-1 is further defined by CPN-PAGE-2. Page 2 has three transitions defined in pages 3 and 4. The "Declaration page" contains definitions of all the tokens, arc inscriptions and functions used in the CPN.

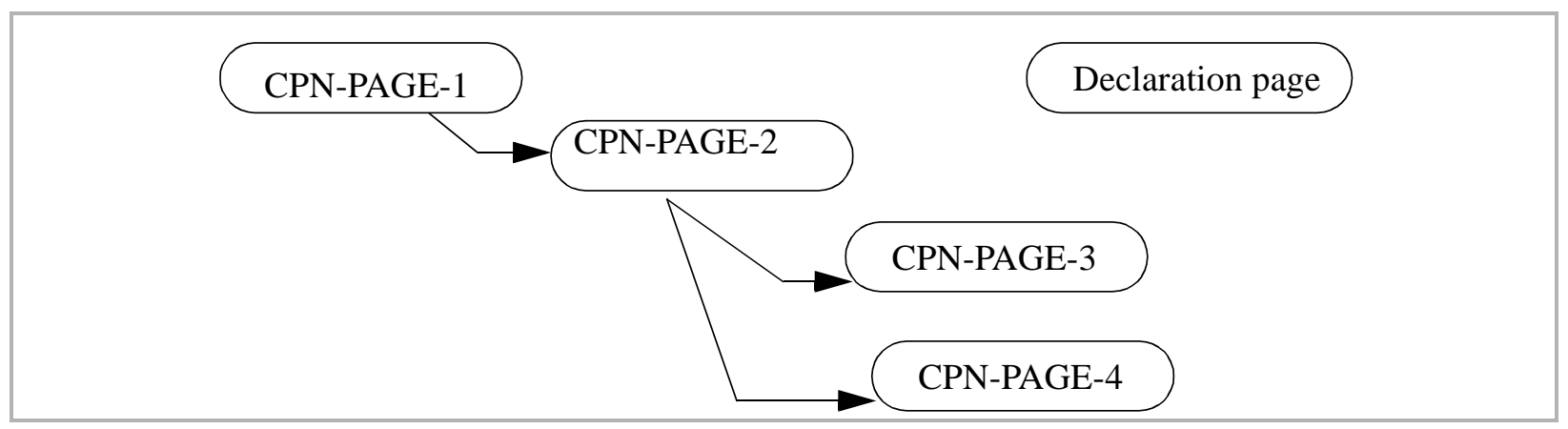

Figure 1-4 Description of CPN Hierarchy 
A CPN is a tuple $S$ : a finite set of pages such that each page $s \in S$ is a $C P N=\left(\sum_{s}, P_{s}, T_{s}, A_{s}\right.$, $\left.\mathrm{N}_{\mathrm{s}}, \mathrm{C}_{\mathrm{s}}, \mathrm{G}_{\mathrm{s}}, \mathrm{E}_{\mathrm{s}}, \mathrm{I}_{\mathrm{s}}\right)$, where:

- $\quad \sum$ : finite set of non-empty types (or color sets)

- $\quad$ P: finite set of places

- $\quad \mathrm{T}$ : finite set of transitions

- A: finite set of arcs

- $\quad \mathrm{N}$ : node function

- $\mathrm{C}$ : Color function $(\mathrm{P} \in \Sigma)$

- G: guard function. It is defined from $\mathrm{T}$ into expressions.

- $\mathrm{E}$ : arc expression function. It is defined from A into expressions.

- I: initialization function. It is defined from $\mathrm{P}$ into closed expressions.

This description of CPN will help in defining the mapping rules from SART to CPN

(Chapter 4) and from DMM to CPN (Chapter 5). The following section gives an overview of SART notation and some key elements considered in this work.

\subsection{SART notation for generating specifications}

SART has been implemented in several COTS tools like Teamwork ${ }^{\mathrm{TM}}$ by Sterling Software Inc., and StPTM (Software through Pictures) by Aonix Inc. Teamwork was extensively used in this work. This section describes SART as implemented in Teamwork. StP uses similar notation with slightly different graphical symbols, e.g., a dashed bubble for control specs verses a solid perpendicular line in teamwork. Both of these tools use a similar symbol for a process (a solid bubble). Teamwork has another feature that is very helpful in automated mapping process. A 
teamwork/SART model can be saved in CDIF (Case Data Interchange Format). The CDIF files are used as input to the mapping utilities to create models for the target environment (such as Design/CPN). CDIF is a family of standards that lays out a single architecture for exchanging information between modeling tools, and between repositories, and defines the interfaces of the components to implement this architecture. More information on CDIF is available at "http:// www.eigroup.org/cdif/intro.html”.

SART notation hierarchically divides a software system based on its functions. The topmost layer in the hierarchy (or the most abstract representation) consists of what is commonly termed as the context diagram (CD), the highest level Data Flow Diagram/Control Flow Diagram (DFD/CFD) in the model. It describes the boundary of the software under analysis as well as the external interfaces and the external entities. The software under consideration is shown as a single process (bubble) in the middle surrounded by input/out data and control flow (representing the external interfaces) from/to the external entities represented by rectangles (termed as terminators) as discussed in the following subsections.

The next level in an SART model hierarchy is a data flow diagram (DFD-0) that represents the major functions outlined in the functional requirements. These functions represent a top level decomposition of the software under development. Consequently the whole DFD-0 is viewed as the child of the process or the bubble representing the system in the top level (i.e., process 0 in the CD). Each bubble in DFD-0 (numbered 1,2,3, etc.) has either its own child DFD or a P-spec sheet (see below) describing it in more detail. DFD-1, the child DFD of process 1 in DFD-0, has its processes numbered 1.1, 1.2, 1.3, etc.

DFDs/CFDs model the processing of information in terms of data and control flows; data processing nodes and control nodes (or controllers) represent the data processing functions and the control functions, respectively. Data flow represents flow of data items, received and processed by "processing nodes", whereas control signals are used by the "control nodes" to control the data processing activities in the system at hand. 
Process specifications (P-specs) describe the details of the DFD defined data processing nodes. These specifications consist of pseudo-code scripts or just plain text, explaining how output flows of a particular processing node is generated from its input flows. Control specifications (C-specs) describe the details of control nodes (or controllers) in a CFD. These specifications define the behavioral (or state) model of the controllers and specify how the output control flows are obtained from the input control flows. They also specify when the data processing nodes are activated or deactivated. Data dictionary defines all the data items and control signals in the system. It contains text defining each item and their value range. More detail about these artifacts is given in the following subsections.

\subsubsection{Data Flow and Control Flow Diagrams (DFDs/CFDs)}

DFDs specify the data processing activities in a system. CFDs specify the flow and the processing of control signals in the system. DFD consist of processing nodes, data flows and data stores; whereas CFD consists of control nodes, control flows and control stores. DFD and CFD are tightly coupled since the control nodes in a CFD controls the activation of processing nodes in its corresponding DFD, that in turn might produce a control flow used as an input for a control node in the CFD. The two diagrams can be drawn separately for very complex systems. However in hierarchical analysis, the emphasis is to keep the combined DFD/CFD simple at any given level of hierarchy and divide the complexity among several lower level DFD/CFD. The notation for DFD/CFD consists of the following components.

External entities (represented by rectangles, also called terminators) specifying entities outside the boundaries of the system to be modeled. These entities are either hardware or software components or other systems that produce (or consume) data items and control signals processed by the modeled system. Examples of external entities are operators, sensors and actuators. Processing nodes (represented by circles or bubbles) specify processing functions within the boundaries of the modeled system. The naming of nodes should represent the actions performed 
and should always start with a verb followed by an object on which the verb acts. A processing node operates on a set of input data items or control signals and produces at its output data items or control signals. A processing node represents a transformation at a given level of abstraction. If the transformation is complex, the processing node is associated with (i.e., is a parent of) a lower level DFD/CFD (also called the child diagram) representing the transformation in a finer level of detail. Control nodes (vertical bars) represent controllers that activate processes specified by processing nodes in the same diagram, and consume and generate control signals (or control flows). A control node symbolizes a part of the control section in any computing system taking care of controlling the data processing elements in the processor. The details of the control process in a control node is specified in another sheet by a state transition diagram and/or a table, (e.g., a process activation table, decision table or a state-event table. Every control node is labeled to distinguish it from other control nodes in the same CFD.

Data flows (represented by solid lines) are directed links originating (or produced) from an external entity, a processing node or a data store and terminating (or consumed by) at an external entity, a processing node or a data store. A data flow may represent a single data item or a group of data items abstracted or combined under one name. A group data flow associated with (input or out of) a parent processing node may be divided into its element data items at the child DFD/CFD. Data flows are described in details in terms of their elements and the values they might have the data dictionary of the structured analysis model. Control flows (represented by dashed lines) are a directed link originating from an external entity, a processing node or a control node, and terminating at a control node or an external entity (a control flow is also allowed to terminate at a processing node only if this node is defined by a lower level DFD/CFD having a control node that consumes the control flow. A data store (two solid horizontal bars) represents a repository ranging from a simple data buffer or queue to a large data base. A control store (two dashed horizontal bars) represents a memory component containing signals used by controllers (as control flows). 


\subsubsection{Process Specification (P-spec)}

Each primitive processing node in a DFD (i.e., a simple processing node NOT having a child DFD in a lower level to specify it in finer detail) must have a P-spec. A P-spec, in essence, is the child of the primitive processing node it describes. It shares the same input and output flows, and specifies how the produced output flows are obtained from the consumed input flows.

P-specs are defined in terms of a textual specification describing a procedural process. Structured English is used to make the text concise with the intent to combine the rigor of a programming language and the readability of English. P-specs can also contain mathematical equations and illustrations such as tables, diagrams and graphs to ensure specifications are complete and unambiguous.

\subsubsection{Control Specifications (C-specs)}

A control node (or a controller) in the CFD is specified by a C-spec that determines in detail how and when the output control flows of the control node are asserted. It also specifies the currently activated processing nodes in the corresponding DFD. The activation signals to the processing nodes are not shown explicitly as outputs of the control node. The notations for Cspecs are divided into four types: Decision Tables (DTs), State Transition Diagrams (STDs), State/Event Matrices (SEMs) and Process Activation Tables (PATs).

The choice of a particular type or a combination of types depends on the application and problem at hand. DTs specify combinational controllers that assume only one state. A STD or a

SEM is used to specify sequential controllers consisting of several states. PATs are used to specify activation of processing nodes for combinational controllers with no output control flows (activation signals are not considered to be explicit output flows of control nodes). These types are further described in the following discussion. Due to their wide acceptance in the software industry, only STDs are considered in this work. 
STDs specify controllers consisting of a sequence of states for sequential controls. A rectangle is used to define each state. Directed arcs between rectangles specify transitions from one state to another. A state transition is caused by a specific event consisting of a combination of input control values and produces actions consisting of process activations and a combination of output control values. A PAT can be used with a STD to specify the process activations for the various state transitions.

There are two well known sequential machine models used to define sequential controllers: Moore and Mealy. In a Moore model, the output actions (process activations and output control value) are associated with the states. In a Mealy model the actions are associated with the transitions between states, i.e., with state-event combinations.

STDs can be of the Mealy type where a transition is labeled by the event causing it, followed by a slash, followed by a set of numbered actions. The number given to an action specifies the order in which it is executed (e.g., a value is specified for an output control flow or an activation signal is asserted). STDs can also be of the Moore notation where a state definition rectangle consists of a state name, followed by a slash, followed by the set of numbered actions. It is possible to mix the two notations in one STD, where a hybrid Mealy/Moore model is defined. The Mealy/Moore model is developed depending on whether it is more convenient to specify actions (i.e., generating outputs or activating processes) within the rectangles defining states or specify actions with the arcs representing transitions. Each output action must be specified with a state (or a set of states) or with a transition.

Every STD contains an initial state where the control processes start when the controller is activated. This state is designated by a transition specified by an incoming directed arc with no source or origin, i.e., incoming or originating from the empty space. An important note, concerning process activation signals specified as actions in Mealy STDs, is that activation signals associated with a transition are assumed to continue in effect until the next transition occurs. This means an activated process remains active until the next transition occurs. There is 
no need for deactivation signals since processes are automatically deactivated, unless they are activated again in the next transition. For Moore STDs, a process activated at a given state remains active until a transition to a different state (where the process in not activated) occurs.

\subsubsection{Data Dictionary}

A data dictionary contains the definition of all the data items and control signals. Data dictionary entries, in general, are divided into: primitive data items and compound data items. Primitive entries are items not composed of any other data items. Compound data items may be composed of other compound data items and/or primitive data items. Examples of primitive data items are: a temperature sensor reading, a binary switch reading, operational status or an identification number. Examples of compound data items are an operator command consisting of several different types of commands, or a sensor data consisting of the readings of different sensors. Compound data are needed for the purpose of abstraction to define the data hierarchy making the analysis diagrams and tables easily manageable and readable. The DFD's functional hierarchy usually requires some form of data hierarchy where compound entries are used in the higher levels of hierarchy and primitive items are used in the lower levels.

Primitive items are either continuous or discrete. A continuous item is characterized by its range, resolution and units; whereas discrete items are defined by a set of values or symbols it assumes. Compound items, or complex expressions, can be obtained by combining other compound items as well as primitive items. These items are combined using selection, concatenation and optional operations using BNF notation.

\subsection{UML notation for generating specifications}

UML unifies the Booch, OMT and Jacobson methods. By unifying these leading OO methods, UML provides the basis for a common, OO development method. Booch and OMT are well recognized in the software development community [12]. Realizing this, Rumbaugh and 
Booch developed a UML in October 1994. They invited Ivar Jacobson to bring in his use cases method in 1995 for their unification effort. The result is UML.

UML is a third-generation method for specifying, visualizing and documenting artifacts of an OO system under development. UML represents the unification of the Booch, Objectory and OMT methods. The following paragraphs give an overview of UML. More information is available from UML links on the web site of Rational Software Corporation (www.rational.com).

UML provides support for modeling classes, objects and the relationships among them, including association, aggregation, inheritance, dependency and instantiation. Use cases are supported with scenarios for detailed descriptions of required system behavior. Interaction diagrams graphically model scenarios and can include timing and message synchronization annotations. Finite state machine modeling supports a number of real time features, including concurrency, event propagation and nested states. UML also provides notations and semantics when more complex modeling is required. Two such advanced features are stereotypes and packages.

A stereotype is the meta-classification of an element in UML and identifies the UML element type. For example, predefined UML class stereotypes include event, exception, interface, metaclass and utility. Predefined task stereotypes include process and thread. The primary advantages of stereotypes are:

- it is possible to refer to the type of the element, as in "That class is an Exception class,"

- UML is extensible by a user through the definition of additional stereotypes.

For large-scale development, UML supports the concept of packages, a grouping of inherently cohesive entities. All of the classes in a model can be packaged by the area of concern, such as user interface, device I/O, etc. The implemented code can be packaged into subsystems representing deployed software components. The notation for packages is a tabbed folder. 
Stereotypes clarify the type of package («category» for the class model or «subsystem» for the code model). Packages provide a namespace for the items they contain. This becomes important as a system grows larger and particularly when third-party software is being used. If a name conflict occurs between components, they can be placed into separate packages and referenced with their fully qualified naming scheme.

UML deals with the notions of model and diagram. A model contains all the underlying elements of information about a system under consideration and is independent of how those elements are visually presented. A diagram is a particular visualization of a certain kind of elements from a model. Only a subset of those elements' detailed information is generally visible. A given model element may exist on multiple diagrams, but there is only one definition of that element in the underlying model. UML supports the following kinds of diagrams (each type of diagram captures a different perspective or view of the system's underlying model): class, usecase, interaction (sequence and collaboration), state, component and deployment.

The first four diagrams address elements of a system's logical model. The logical model means the system is being modeled somewhat independently of exactly how actual software components are named and organized. The last two types of diagrams represent more of the physical aspects of the system so they will not be considered for this work. The following sections describe these diagrams.

\subsubsection{Class Diagram}

As with other OO methods, a class diagram is key to a UML model. A class diagram shows the important abstractions in a system and how they relate to each other. The primary elements found on class diagrams are class icons and relationship icons. Individual classes are represented in the UML as solid, outline rectangles with one, two or three compartments. The first compartment is for the name of the class and is required. The second and third compartments are optional and may be used to list the attributes and operations defined by the class. In addition to 
classes, attributes and operations, class diagrams also depict various types of relationships existing between dependent classes. UML allows different kinds of relationships: associations, aggregation, inheritance, dependency and instantiates.

An association represents a structural dependency between objects, generally of different classes. To specify how many instances participate in an association, UML defines adornments for multiplicity. Numbers and asterisks next to the endpoints of the associations specify the potential number of objects participating in the relationship. Multiplicity can be indicated by either a constant (when known) or with a '*' to indicate "many". An unadorned relationship end is assumed to be "unspecified" in its multiplicity; no default value is assumed in UML.

Associations are bidirectional by default. This means an instance of one class can navigate to instances of the other class and vice versa. Navigability is often realized by objects maintaining references between associated objects. When an association is left in its bidirectional form, there exists a circular dependency between the corresponding objects, resulting in a peer-to-peer relationship between those objects. In UML it is possible to "limit navigability" in one direction of the association so as to simplify the implementation and establish a more client-server relationship. In a unidirectional association, UML introduces an arrowhead at the server end of the line segment.

An aggregation is a special form of an association used to show that one kind of object is composed, at least in part, of another. Aggregation indicates the lifetime of the parts are dependent on the lifetime of the whole. This means part-side objects cannot be created unless and until their associated aggregate-side object is created. Similarly, part objects cannot be destroyed by any object other than the aggregate object that created them in the first place. Aggregation can further be refined in UML to denote how the aggregate's containment of its parts is implemented. The default is by reference, meaning the whole object maintains a pointer or a reference to its parts. By-value aggregation has the same semantics as an attribute. A particular aggregation form may be needed when the contained object has a complex structure of its own. 
An inheritance relationship is used when one class shares the structure and behavior defined by another; that is, when one class is a specialization or extension of the other. In UML, the more generalized class is called the superclass and the more specialized class is called the subclass. A subclass inherits all the attributes and operations specified in its superclass, as well as any relationship dependencies the superclass might have against other classes. A subclass may then specialize the implementation of inherited operations, or extend the superclass's structure and behavior by adding brand new data and operations.

A dependency relationship means a client class depends on some service(s) of a server class, but does not have an internal structural dependency against the server. The most common form of general dependency is found when an operation defined in a client class takes on an argument of some other class type.

An instantiate relationship is a special type of dependency existing between a parameterized class and the class created as a result of instantiation. Parameterized classes are templates for regular classes because they are set up to function independently of the type of information with which actual classes will work. Container classes are most often implemented as parameterized classes so they can be written without regard of the type of items being contained. Formal parameters are usually generic class or type names. The result of an instantiation relationship is an instantiated class named with the parameterized class as a prefix. This is followed by an angle-bracketed name representing the actual parameter of the instantiation. An instantiate relationship often provides an alternative to inheritance for constructing reusable components.

\subsubsection{Use-Case Diagram}

Use cases are descriptions of how a system will be used and provide a high level view of the intended functionality of the system. Relatively easy to draw, use cases are convenient when talking with customers. 
A large rectangle shows the boundaries of the system. Smaller rectangles arranged around the system are external entities interfacing with the system. These external entities are modeled as classes, but annotated with a special property called a "stereotype", designating them as actors. An actor initiates a use case or it could be a recipient of the system usage. Ellipses inside the system rectangle indicate the use cases themselves. Some use cases depend on others, as denoted by the dependency relationships between use-case ellipses.

\subsubsection{Interaction Diagrams}

Interaction diagrams are used to model timing and sequencing requirements. Some of the important features of interaction diagrams are: scenarios, sequence diagram, collaboration diagram and message synchronization.

A scenario is a specific instance of a use case. A use case represents many different possible threads of a specific interaction. Each specific thread (through a use case) is called a scenario, a particular path through the system functionality. A single use case represents many related yet distinctly different scenarios. In system behavioral modeling, it is common to depict dozens of scenarios for each use case. UML provides two notations for modeling scenarios: the sequence diagram and the collaboration diagram. Objects are shown on both types of diagrams using rectangles just as for classes. To differentiate between objects and classes, the name in an object rectangle is underlined. The class of the object may optionally follow the object name and a colon.

Sequence diagrams use object icons with vertical dashed lines projected downward on the diagram. The horizontal directed lines represent the messages passed between objects. Time flows from the top of the page downward. Unless specifically annotated, only the sequence of messages is shown, not the exact time. The textual annotations along the left edge of the diagram are optional and referred to as a script. Each statement in the script helps explain one or more messages being passed in the diagram. A script may directly correspond to the actual scenario 
being modeled by the sequence diagram. For realtime designs, exact timing often must be specified. UML allows textual annotations to be added to sequence diagrams when timing is important.

The other notation for modeling scenarios is the collaboration diagram. Sequence numbers are attached to messages to indicate the relative order the messages are sent in the scenario. The arrows show message direction. One can see that sequence progression is more prominent in the sequence diagram, but structure is more obvious in the collaboration diagram. Timing annotations can be added to collaboration diagrams as well.

Message synchronization icons are the symbols used in conjunction with messages to indicate how the concurrent processes are synchronized during a message transfer. Realtime systems often concern themselves with the synchronization of concurrent processes during message passing. UML provides five different icons that can be added to any message for describing its concurrent behavior: Simple call, synchronous rendezvous, timeout rendezvous, balking rendezvous and asynchronous.

Simple-call is a simple message denoting the synchronization either has not yet been specified or is a sequential message (for example, function call semantics). Synchronous rendezvous means the sender waits indefinitely for the receiver to accept the message before continuing on with its processing. Timeout-rendezvous indicates the sender waits for the receiver to be ready for the message up to some fixed period of time before aborting the message transmission process and continuing on with its processing. Balking-rendezvous means if the receiver of the message is not immediately ready to accept the message, the sender aborts the message and continues. Asynchronous means the sender sends the message immediately and continues on with processing without waiting for the receiver to acknowledge its readiness for receiving the message. 


\subsubsection{State Diagram}

UML state models are based on finite state machines using an extended Harel state chart notation. The features supported are: guards on transitions, propagated transitions, actions on transitions, actions on a state entry, activities occurring as long as a state is active, actions on state exit, nesting of states and concurrency. The UML syntax for transitions is:

event (arguments)[condition]^ ${ }^{\wedge}$ target .sendEvent(arguments)/operation (arguments)

Each of these fields is optional, even the name may be omitted when it is clear when the transition will be taken. The event is the name of the transition. Often this is the only thing specified for the transition. The transition name has an optional argument list to indicate when data is present in the transition, such as an error code or a monitored value. This argument list is enclosed within parentheses like a standard function call. A guard condition is shown in square brackets. A guard is a condition to be met before the transition is taken. Target is an object receiving events from the sendEvent list, a comma-separated list of events, directed toward a given target object, each with possible arguments. Such events will be propagated outside of the enclosing object as a result of this transition.

This is largely how concurrent state machines communicate, allowing a transition in one state machine to affect other concurrent state machines. The operation list specifies a commaseparated list of functions (each with possible arguments) to be called as a result of the transition being taken. Within states, both entry and exit actions, as well as an ongoing activity, may be

specified. An entry action is a function called when the state is entered. An exit action is a function executed when the state is exited. Activities denote processing that continues until completion or until interrupted by a transition.

\subsection{Summary}

This chapter gave a brief overview of IV\&V, and the importance of dynamic simulations 
of the customer supplied artifacts. CPN and the associated tool (Design/CPN) are described as possible environment suitable for carrying out dynamic simulations. SART and UML are the two notations (described in the last two sections of this chapter) are widely used in the industry. These two notations will be used in the rest of this work as examples of customer supplied artifacts. This description will help better understand the problem statement and research objectives in the following Chapter 2. 


\section{Chapter 2 Problem Statement and Objectives}

\subsection{Problem Statement}

The dynamic analysis of software system specifications can provide more insight to an IV \&V analyst during early phases of the software system development. This is accomplished by measuring performability, risk assessment, and dynamic complexity based on semantics extracted from requirements analysis models. A challenge for IV\&V teams is to find ways for developing dynamic models of software systems during the requirement analysis phase. For an IV\&V analyst, requirement specifications may be available from the developer as models based on SART or OO. These models have to be mapped to representations suitable for dynamic analysis. An example of such an environment is $\mathrm{CPN}$ (Section 1.4). CPN is useful for performing dynamic analysis [37], supported by a COTS tool (Design/CPN).

The problems can be stated as: given SART or UML specification artifacts (generated using CASE tools) for large scale industrial systems:

1. Develop methods to map these artifacts to specification models based on CPN, for dynamic modeling, such that,

i. The CPN models should capture the dynamic behavior.

ii. The $\mathrm{CPN}$ model components are to be easily traced to specification components in the original artifacts (in SART or UML).

iii. The models can be used by analysts to conduct scenario based simulations.

2. Implement such methods on a component of a large scale software system. The example of such system considered for this work is Flight Operation Segment (FOS). Chapter 6 gives more detail of this example and presents further analysis. 
The literature survey related to SART and UML is given in Chapter 3. SART was selected because it is being used in large projects, including the International Space Station and X33 (space shuttle of the future). In the OO domain, many notations are in use; for example, OMT, Booch, Shlaer-Mellor and UML. To limit the work at a manageable level, only one of the notations for the $\mathrm{OO}$ models, i.e., UML is used in this work.

CPN was selected as it is suitable for modeling concurrency and can easily be used in reachability analysis, performability analysis and risk assessment. CPN tools usually have a graphical interface and CPN models can be arranged in a hierarchical fashion. These two characteristics match well with the modeling techniques being used in the industry. There are workshops being conducted for Design/CPN and a large body of work is available related to this tool. More information is available from the official web-site of Design/CPN at "http:// www.daimi.au.dk/designCPN/".

The process of generating formal specifications consists of mapping the semantics of the SART or OO models to dynamic models. As is evident from the state of practice in software modeling notations, there will always be a plethora of different techniques and notations in use. This makes the mapping process very difficult and complex. There is a need to outline the semantics of these models. These semantics can then be used to construct dynamic models. For a typical, large scale complex software system, the models are built in a hierarchical fashion. Each level in the hierarchy describes a set of features (or functions) related to the system under development.

There is a need to develop a process dealing with the semantics of SART as well as UML. This process can be implemented using an integrated tool environment facilitating the flow of semantics information from the developer tools to the IV\&V tools. An important link in the integrated environment is a set of utilities to translate the information from the developer's models. The translated information can then be used by the IV\&V tools for building dynamic models. 


\subsection{Research Objectives}

The objective of this work is to develop methods and techniques for generating verification and analysis models from notations used for large scale software system specifications. The models so generated can be used by the analysts to detect potential problems and prevent these problems from becoming part of the design. Development of verification and analysis models depends on the type of specification notation. There are two broad categories of these notations: Structured techniques (SART) and OO techniques (UML) as described in Chapter 1. The research objectives based on these categories are:

- Develop a methodology for obtaining CPN verification and analysis models from specifications based on SART,

- Explore techniques for developing dynamic models for analysis and verification of OO analysis specifications.

The proposed process for developing verification and analysis models consists of many steps, primarily dependent on the semantics of a specification notation. The initial steps for SART based specifications are:

1. Analyze semantics of SART for generating specifications for large software systems.

2. Develop mapping rules for translating semantics of SART models to CPN models.

3. Implement mapping rules (from Step 2) by developing a translator utility based on CDIF (Case Data Interchange Format).

4. Develop a process to integrate IV\&V tasks of risk analysis and performability analysis, with the translator utility (from Step 3).

5. Illustrate the proposed process (from Step 4) by applying it to a large scale software 
system.

The following steps lists the objectives for UML analysis models:

1. Analyze semantics of the UML technique based on the concept of meta-modeling.

2. Develop dynamic metamodels to capture real time behavior of UML based specifications.

3. Develop mapping rules for translating dynamic metamodel semantics to Design/CPN notation.

The goal of this work is to contribute to the state of art in software IV\&V. For developers' models based on SART, the process maps hierarchical requirements models developed in SART notation to hierarchical models in CPN notation. This approach will be implemented using the following tools:

- $\quad$ Teamwork for SART models (see Section 1.5),

- $\quad$ Design/CPN for CPN models (see Section 1.4).

Figure 2-1 shows the process where semantics of the developers' models are mapped to the analysis and verification models.

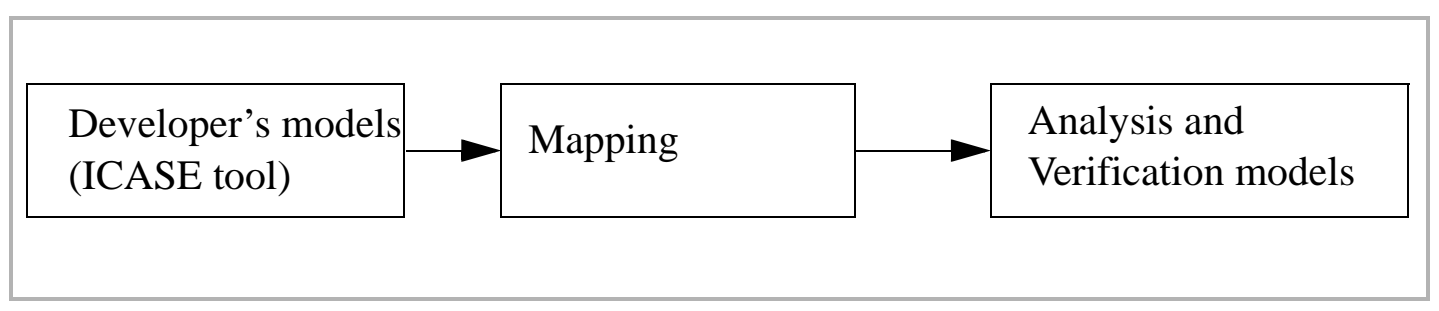

Figure 2-1 The process of mapping SART specification models to CPN models.

Related work presented in Section 3.1 shows the process of translating semantics is non- 
trivial for large software systems. Integrated tool environment can facilitate the flow of semantics information from the developer tools to the IV\&V tools. Such environments may need utilities for extracting semantics information from the developer's models. The extracted information can then be used by the IV\&V tools for building dynamic models.

In summary, this work develops frameworks for generating dynamic models from SART as well as UML models. The following chapter describes related work and contributions of this work in the light of research objectives stated above. 


\section{Chapter 3 Literature Survey}

This work draws from two research areas of software systems analysis and design. The first area is based on generating formal, executable models of software systems. The second is based on managing multiple views of a software system as metamodels. This chapter describes some previous work in these areas. The existing work for generating executable models can be divided into two main groups. The first group deals with developing executable models from SART models. The second group deals with generating executable models from one of the OO models. Both of these groups rely on manual methods for developing executable models that make it difficult to adapt such techniques to large scale systems.

\subsection{Formal specifications of software systems}

The literature ([1]-[6], [13]-[20]) contains a large body of work on software systems analysis based on mapping informal specifications to formal models. Examples from four different approaches are presented below.

- CPN and verification of systems,

- Graph Grammar for Extracting Syntax and Semantics,

- Translating SART to HLTPNs (High Level Timed Petri Nets),

- Translating SART to Calculus of Communicating Systems (CCS),

- Formalizing and Integrating the dynamic model within OMT.

\subsubsection{CPN and Verification of Systems}

Mikolajczak, et al. [21] proposed an approach to OO software design using CPN as a 
graphical modeling tool with formal semantics and substantial simulation capabilities. He argued that CPN conceptual mechanisms can be applied effectively for the same purposes and one can use simulation facilities of the Design/CPN software package to verify important system properties, to make design/implementation decisions and to debug the design. An Automatic Teller Machine (ATM) was used as an example to explain the method. This example covers a class of highly interactive embedded systems with potential concurrent/parallel/distributed implementations. The technique does not suggest ways to deal with large scale software systems.

Jorgensen, et. al. [22] presented abstractions supporting distributed program execution in the OO language BETA. A BETA object for one computer may invoke a remote object, i.e., an object hosted by another computer. Formalism of CPN was used to describe and analyze the protocol for remote object invocation. In the first phase, a model was built to describe, understand and improve the protocol. Remote object invocation in BETA is modeled on the level of threads (lightweight processes) with emphasis on the competition for access to critical regions and shared resources. In the second phase, the model was analyzed, leading to a proof the system has a set of desirable properties, e.g., absence of dead locks. The technique used in this paper is suitable for very small scale systems. Jorgensen, et. al. fail to show their method is scalable to large systems.

Mortensen, et. al. [23] described a modeling project to improve a nuclear waste management program for the permanent disposal of nuclear waste. SART was used to provide a work-flow description of the functions to be performed by the waste management program. This description was then translated into a number of CPNs corresponding to different program functions, where additional behavioral inscriptions provide basis for simulation. Each of these CPN was simulated to produce timed event charts that were useful for understanding the behavior of the program functions under different scenarios. In the final phase, all CPN models were linked together to form a single stand-alone application useful for validating the interaction and cooperation between the different program functions. A technique for linking executable CPN models is developed for supporting large modeling projects and parallel development of 
independent CPN models. Mortensen's method relies on a manual method of mapping where the IV\&V analyst has to know the intimate details of the developer notation. The technique is good only if the analysts and developers are working in the same office.

Rasmussen, et al. [24] presented an industrial use of CPN in designing a security system. An animation utility was developed making it possible to perform CPN simulations. Occurrence graphs were used for debugging the CPN. In this way, a series of errors in the model were found and corrected. CPN design was used as a specification of the implementation of the security system. Therefore, finding errors by means of simulations and occurrence graph analysis reduced the amount of errors in the final implementation, thus improving the quality of the software. Rasmussen, et. al. didn't use a mapping from SART or OO models. Instead they developed the CPN models directly from the initial specifications of the system.

The following section describes a technique for generating executable specifications based on abstract syntax graph grammar and semantic graph grammar.

\subsubsection{Graph Grammar for Extracting Syntax and Semantics}

Pezze [13] presents formal specification methods applicable to large software systems based on Abstract Syntax Graph Grammar (ASGG) and Semantic Graph Grammar (SGG). He contends there is a problem in using a dual language approach. A dual language approach conjugates informal widely used front-end notations (e.g., SART) with rigorous and formal models, e.g., HLTPN. These specifications (given in terms of front-end notations) are mapped to formal models. The problem is the same notation is often used with different interpretations in different organizations and sometimes even within the same organization or development team. Fixed, rigorous interpretations can meet the needs of a specific application domain, but they fail to provide general solutions. Pezze addresses these problems by proposing an approach based on ASGG and SGG. The two grammars, i.e., ASGG and SGG, define the abstract syntax and the semantics of a graphical front-end notation. Each ASGG production corresponds to a SGG 
production. User modifications of the front-end notation are captured by means of ASGG productions. The associated SGG productions describe how to automatically update the formal kernel model.

The proposed method was applied to the models based on structured analysis. But the method needs to do more work to make these techniques suitable for COTS tool environments. They are also planning to consider OO models for their future work.

\subsubsection{Translating SART to HLTPNs}

In [19] and [20] Pezze, Elmstrom and Lintulampi presented semantics mapping rules to generate HLTPN models from the Ward and Mellor SART notations and later modified by Hatley and Pirbhai that is currently supported by most ICASE tools. The approach in their work is to generate petrinets not visible to the analyst. Their objectives are to formalize the SART notation and produce an environment supporting the execution of heterogeneous models where parts of the model are implemented in $\mathrm{C}$ code. The drawback of this approach is the complexity of the HLTPN models obtained. The mapping rules presented produce much more complicated HLTPN models from a relatively simple SART model.

While HLTPN models have been proposed to integrate the functional and time aspects in a semantically precise way, the author of this work believes Design/CPN adopts a simple time model added to the CPNs formalism. Therefore SART to CPN translation is more effective in developing dynamic models. Another important aspect to note is that HLTPN has been proposed to model the detailed realtime properties of systems and hence are more suitable to be used at the detailed design or code level rather than at the requirements analysis level.

\subsubsection{Translating SART to Calculus of Communicating Systems (CCS)}

Hooker, et. al. [15] describe integration of Ward-Mellor SART notation and the Value Passing Synchronous Calculus of Communicating Systems (SCCS-VP) notation. This is an 
extension to SCCS (Synchronous Calculus of Communicating Systems) notation. The process of integration involves three major steps. In the first step SART models are used as an input to the SF (Semantic Function) implementation. The output of SF is an SCCP-VP program. In the second step the SCCS-VP program is translated to a basic SCCS program. In the third step a CWB (Concurrency Workbench) is used for the model checking and simulation of SCCS programs. Hooker, et. al. have mentioned limitations in this approach. First there are a lot of manual substeps involved. Secondly the tools used are not COTS. The third problem is that there is no tool available to analyze and simulate SCCS-VP programs. That is why SCCS-VP programs have to be translated to a basic SCCS before CWB can simulate it.

Another example of the use of CCS is the work by Krishnan [26]. He discussed the possibilities and limitations of using CCS for the purpose of software system analysis and showed how a formal description can be generated from existing informal documents. The disadvantage, as he pointed out, was that only small, restricted sub-systems can be analyzed using this approach.

\subsubsection{Formalizing and Integrating the Dynamic Model within OMT}

Cheng, et. al. [1] presents a formal model for both the object and dynamic models and their integration. The formal model is described in terms of a specification language LOTOS [25]. Cheng shows the lack of a well-defined semantics for the integration of the three OMT models hinders the overall development process, particularly during the design phase.

It is difficult to construct a formal specification directly from an informal, high-level requirements document. Formal descriptions potentially involve considerable syntactic detail and require careful planning and organization on the part of the analyst to develop modular specifications.

A complementary approach to describing requirements is the use of graphical modeling notations. OMT is a good example of such notation. Wang, et. al. [1] argues that to effectively use 
OMT, particularly for design purposes, there must be a well-defined method to integrate the three models. Otherwise, OMT is only a combination of three separate, independent models providing little more than intuitive diagrams. The integration of object, dynamic and functional models can play a key role in OMT. Unfortunately, the integration is currently not well-defined in [1]. Also OMT is being discarded in favor of UML.

\subsection{Metamodels}

Metamodels are models of models. Metamodels are developed using semantics extracted from other models. Figure 3-1 shows the process of combining semantics of model A and model B to form a metamodel. This is a level-one or $\mathrm{M}_{1}$ metamodel as suggested by Jarke, et. al. [27]. A more abstract form of metamodels $\mathrm{M}_{2}$ can be obtained by combining many $\mathrm{M}_{1}$ metamodels.

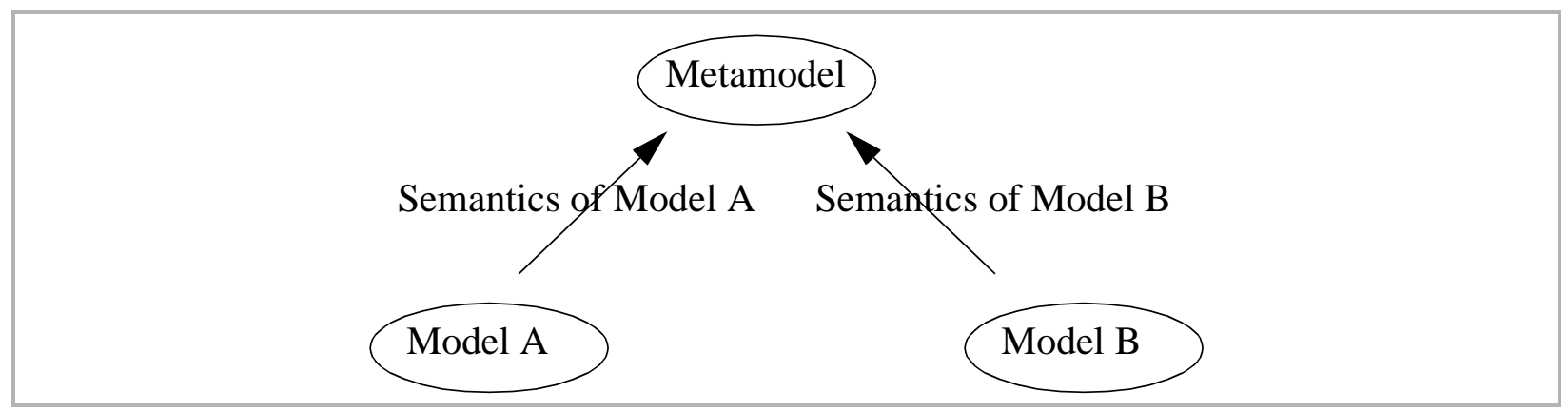

Figure 3-1 Metamodels verses models

In software development domain, metamodels can be formed by combining models of one development phase (such as requirement analysis phase) or many phases (such as requirements and design phases). An even more comprehensive metamodel may include analysis models, design models, as well as models of the tool environment.

This work is limited to the early phases of the software development lifecycle. Therefore metamodels are focused only on the requirement analysis phase such as UML analysis models. Four of the important aspects of metamodels are described in the following subsections. 


\subsubsection{Using Metamodels to address multiple requirements perspective}

Jarke, Nissen, et. al. [27] present an approach for developing requirement documents for software systems. The approach is based on extracting information from several different perspectives. Examples of such perspectives are user perspective, developer's perspective, tester's perspective, etc. These perspectives use different notations for developing models. Jarke shows how information contained in these models can be extracted into a single framework called metamodel (or a model of models).

Metamodels were applied for developing requirement documents. This is based on capturing requirements from multiple information sources. The method consists of team workshops as well as individual interviews. Facilitators are used to list the conflicts among the perspectives of different stakeholders. These conflicts are then used to elicit other requirements.

This method creates visual, partially overlapping and conflicting requirements perspectives. These perspectives are analyzed for possible incompleteness, inconsistency and conflicts. Once the analysis is complete, these perspectives are used to create the requirement documents.

This method is used before traditional analysis and design, but it still provides a framework that is useful in developing techniques for generating executable specifications during the analysis phase.

\subsubsection{Problems in Metamodeling}

Based on the CDIF standard, Artsy [28] proposes an integration of OO tools by applying metamodeling to $\mathrm{OO}$ models. This metamodeling framework allows comprehensive representation of diverse systems, including complex structures, processes, rules and semantics. The development of metamodeling framework has encountered four major problems: 
1. Most $\mathrm{OO}$ models are not expressed in an explicit and clear metamodel. Without precise and accurate specification of a method, it is difficult to understand, accept and adhere to the method. The lack of precision also hinders the interoperability between tools.

2. Very few tools implementing an OOA\&D (OO Analysis and Design) method have an explicit metamodel. Without such a model, the tool's user cannot know precisely how accurately the tool views or implements certain concepts.

3. Even when there are metamodels for OO models and tools, they usually are expressed in particular notations. These notations differ not only graphically (for example, boxes versus clouds), but also semantically (for example, the notations of OMT versus Shlaer-Mellor). Similar names may mean different things, e.g., class versus class, where one implies an analysis-level type definition and the other the implementation of an object. Different names may mean similar concepts, e.g., class, object type and object.

4. Similar to metamodeling a system and an application, there is the problem of how to represent metamodels for OO models and tools. Which meta-metamodeling concepts can express such diverse notations and different meanings?

For Problems 1 and 2, the CDIF OOA\&D Working Group is cooperating with OO modeling teams. About four metamodels for the more popular OO models are under development. This is a complex task because some of the concepts are vague in the literature. Even the developers are not sure about their correct meaning or intended usage. This task will also resolve Problem 3 as those method-specific metamodels are expressed using the CDIF standard. Using this standard, the same graphical notations and naming conventions help model all the methods. For the Problem 4 it is suggested to select the CDIF flexible structured metametamodeling framework. This is because of its ability to support coexistence with and migration 
from other methods.

One important problem in developing the synthesized standard metamodel is consolidating the metamodels for two main classes of OO models. The first class of OO models deals with more OO "purist" methods in which everything is an object, type or class. The second type distinguishes between objects and other things such as attributes, operations and parameters. Work is underway for synthesizing the metamodels of these methods. It is still an open problem to find an approach that can combine these methods and synthesize their semantics.

Nissen, et. al. contends that even though the reported experiences do not constitute a full scientific validation of the approach, one can draw some lessons. The main lesson learned is that this metamodeling approach is commercially feasible. It does relieve the problems associated with combining informal teamwork-oriented methods and formal, automated analysis tools. It is a big advantage to be able to tailor the metamodels and the integration strategies to the problem at hand.

\subsubsection{OO and Metamodeling}

Odell [29] describes different problems encountered while building metamodels for OO models. Most CASE tools maintain models and metamodels as physically separate levels. This results in a need to modify the metamodel if an analyst wants to change the kinds of object types definable at the model level. This is visible in a situation where an analyst decides to use the functional decomposition of processes in an $\mathrm{OO}$ modeling environment. Analysts must change the metamodel to support tree structure relationships among process types. While this separation of model and metamodel seems sensible, the rigid physical separation can be difficult because of the reasons listed below:

- The rigid physical separation in a CASE tool usually means the model level cannot reference instances in the metamodel level. Technically, an object can be specified at the 
model level. However, most CASE tools consider a model level instance named object to be different from a meta-level instance with the same name.

- Modifying separately maintained metamodels is often impossible. A software vendor may provide no metamodel customization features or may consider the contents of its metamodel to be proprietary. Worse yet, the metamodel may only exist implicitly as program code - making it extremely difficult to modify and extend.

- Rigid, three-level modeling falters when the bottom level (i.e., the data and process level) also has instances.

Odell points out that modeling in rigid, physically separate levels can create problems. The remedy of this problem is to model within a single framework. The benefits of the single framework approach are:

- Model maintenance is performed on one coherent and integrated model, not fragmented by levels.

- Object types and instances share the same model, so a two-way knowledge of their association is not lost as they were with segregated levels.

- Any number of instantiate levels is possible since a rigid number of levels is not required.

An additional advantage is that a single framework model can be used to describe itself, as well as the enterprise. If a model is sufficiently descriptive, it should be able to specify its own object types. A small subset of the model can then describe a much larger subset, that can be propagated to an even larger subset, and so on. Furthermore, any changes to the model can be rigorously specified by and within the model.

Odell cautions that the single framework approach described above only works when kernel, metamodel primitives are defined in a precise and consistent fashion. Without agreed upon 
sets of symbols and well formed rules and axioms, the results would be an informal collection of different models.

\subsection{Summary}

This chapter gave a brief overview of related work. The first part of the chapter described different methods of generating dynamic models. These methods are more suitable in the developers' domain as opposed to the IV\&V domain. This is because of their lack of support of integrated environments and the lack of COTS simulation tools, etc. The framework presented in the following Chapter 4 avoids these pitfalls and presents techniques for developing dynamic models from SART models. The second part of this chapter described Jarke's method of developing requirement documents based on metamodels. He shows the use of metamodels in capturing information from different perspectives. The use of these metamodels is restricted to the single domain of requirement elicitation. This restriction helps avoid problems described by Artsy and Odell. Such problems arise because metamodels can become very complex when they try to model all the phases of development lifecycle. This work follows Jarke's approach and restricts the proposed metamodels to requirements analysis domain. The resulting framework is presented in Chapter 5. 


\section{Chapter 4 Dynamic Analysis of SART Models}

This chapter presents the first area of this work's contributions to the state of practice in the SART based software specifications. For software system specification analysis, SART is one of the widely used notations. The following sections describe mapping rules for SART to CPN mapping. Each set of rules is accompanied by a small example. A large scale system is analyzed using this technique in Chapter 6.

The objective of this mapping is to produce CPN models that can be used, refined and parameterized for dynamic analysis by the analyst. These models must start at a comparable level of abstraction and complexity as the developer's models. There is an almost one-to-one mapping of developer's model objects to V\&V model objects and hence they can also be scaled to model large software systems. CPN models can be used for reachability and deadlock analysis, performance analysis, performability analysis, reliability analysis and in general can be used to verify the information system properties as reported in a number of publications ([19], [20], [30][36]). Some of the properties that can be verified using petrinets are:

- derivability and consistent definition of outputs,

- performability of processes,

- application dependent properties of a concurrent system, such as timing deadlocks,

- Metrics such as dynamic complexity, for dynamic behavior of concurrent systems,

- Identification of high-risk scenarios of complex systems using reachability analysis etc.

The proposed process for mapping SART models to CPN models consists of different steps as shown in Figure 4-1. The process consists of five main steps. In the first step, software system's models are received from the developer by the IV\&V team. The models follow notation 
of teamwork/SART tool. These models are converted to a textual form based on CDIF standards in step 2. The CDIF conversion tools are packaged along with the rest of teamwork environment. Other SART tools like StP follow the same practice of providing a CDIF translation tool in their tool package. More information on CDIF is available at "http://www.eigroup.org/cdif/intro.html" and Teamwork reference manuals. A CDIF based representation of SART notation is described later in Section 4.2. For step 3, an STU (Semantics Transfer Utility) was developed using the mapping rules proposed in this work. The mapping rules were implemented using lex and yacc utilities on a unix platform. STU uses CDIF files as its input and converts their semantics to a Design/CPN compatible text file. This file in turn is used by the Design/CPN to generate a primitive CPN model in step 4. The primitive model is parameterized in step 5. In the next step 6, simulations are performed using these models.

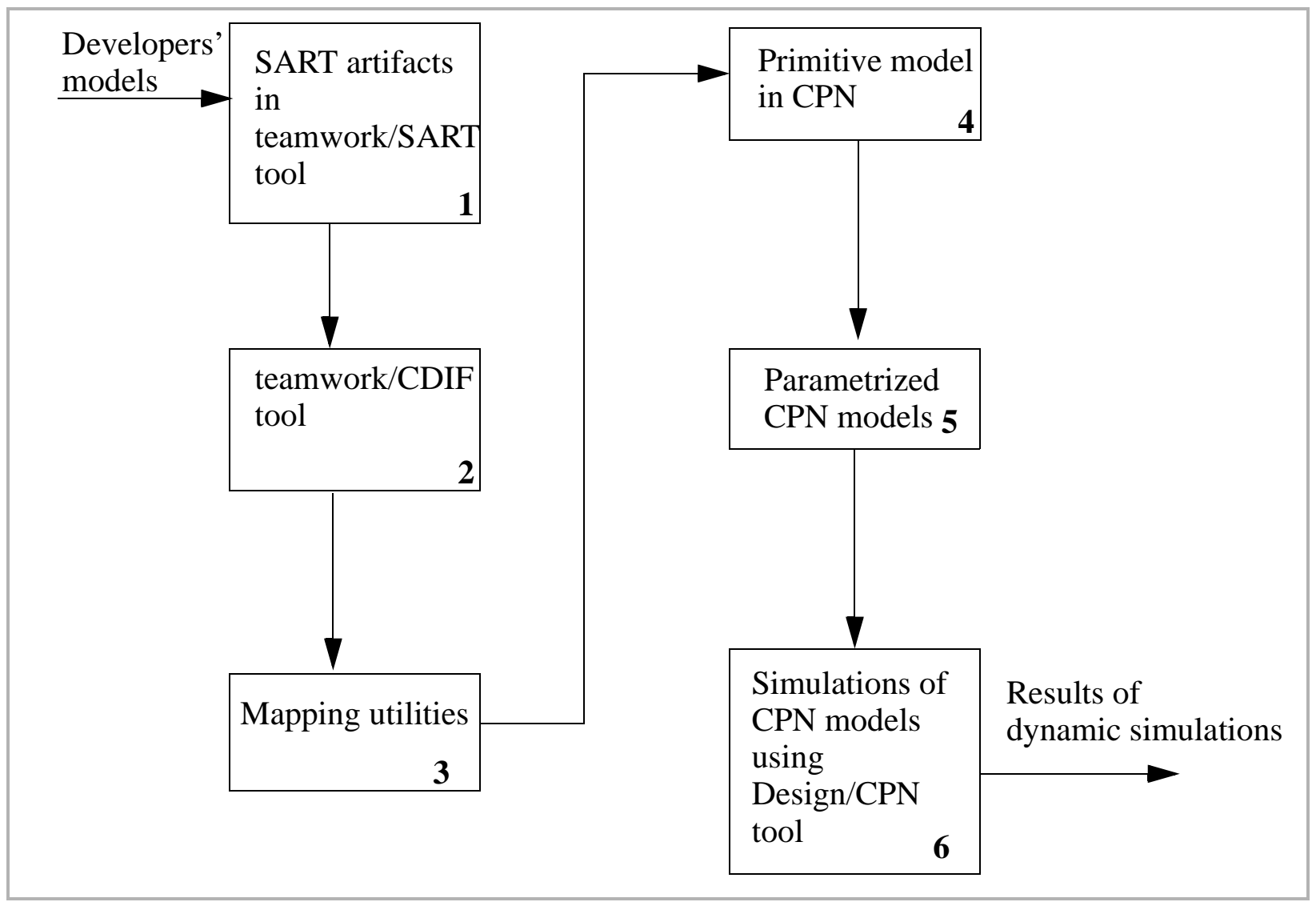

Figure 4-1 Proposed process for dynamic analysis of SART models 


\subsection{Comparison of the SART and the CPN Environments}

The SART model components are shown in the Figure 4-2. The components of an SART model consist of Data Flow/Control Flow Diagrams (DFD), Control Specifications (C-Spec), Process Specifications (P-Spec) and Data Dictionary Entries(DDE). The data flow diagram at the highest level of abstraction is known as the Context Diagram. This diagram allows three types of objects namely a bubble, terminators, data and control flows. The single bubble represents the whole system. Terminators represent the external entities that send or receive data or control signals from the system. The data flows connect the terminators and the bubble.

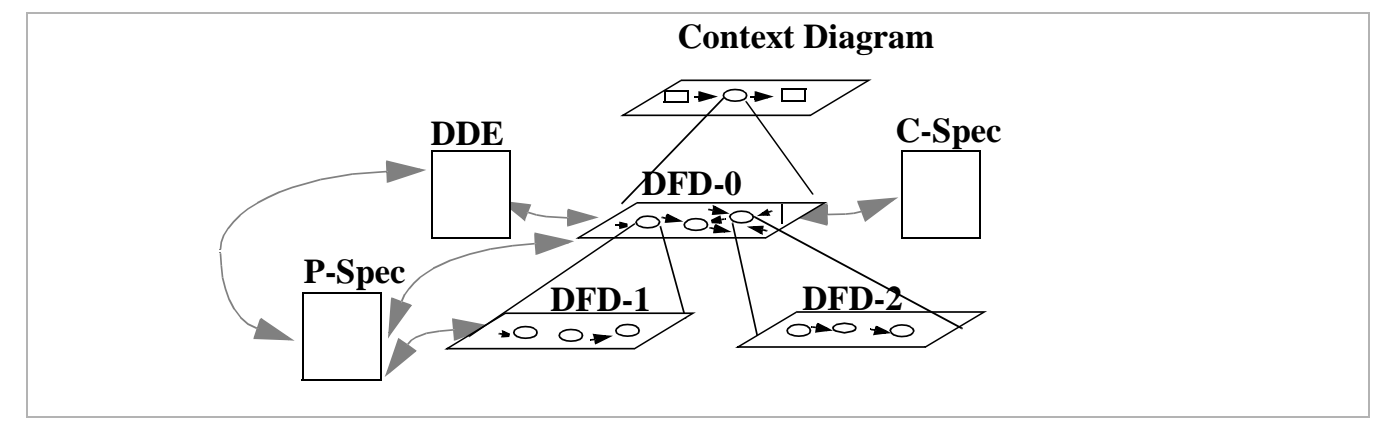

Figure 4-2 Components of SART and their relationship with each other

The bubble on the context diagram is decomposed into more bubbles or processes on DFD 0 . Each of these bubbles will be numbered as 1 or 2 etc. Its up to the analyst to see if any of these processes are primitive. For a primitive process, a P-Spec (or process specification) is defined. If a process is not primitive, a lower level DFD will be used to define it further. For example in the Figure 4-2, two processes in DFD 0 are defined by the lower level DFD 1 and DFD 2. This step are repeated until the analyst reach the primitive level for every process in the model. Definitions for data flows, control flows and stores constitute the data dictionary for a given model. The fields of the data dictionary corresponding to individual flows or stores, are called DDE (Data Dictionary Entries). Also a data flow diagram may contain a Control Specification (C-Spec). The 
Control Specifications are used to define process activation or handling control flows. A vertical bar on the DFD represents a Control Specification. The Control Specification is further defined on a separate sheet in the SART model. Several representations are in use for defining C-Specs. Some of the examples are State Transition Diagram, Process Activation Table, Decision Table etc. In this work, only State Transition Diagrams are considered to limit the scope of this work.

The CPN Modeling environment is also hierarchical. A high level mapping from SART to CPN is shown in Figure 4-3. This The CPN-PAGE-1 is superpage for the rest of pages named CPN-PAGE-* (where * is an integer 2 through 5). Inversely CPN-PAGE-2 is subpage for CPNPAGE-1. Each page in this case has an associated SART object (from Figure 4-2) that is shown just outside the CPN-Page's oval representation. The CPN-PAGE-1 is mapped from the Context Diagram. Similarly CPN-PAGE-2 is mapped from the DFD 0. Other DFD levels are mapped to corresponding CPN pages as shown in the Figure 4-2. For the CPN model, the entries in the declaration page are derived from the DDEs of the SART model. A CPN page may contain objects such as transitions, places and arcs. A place is used to hold data tokens. The number of tokens present in the places of a CPN model at any given moment determine the system state. Transitions are used to show the change in the system state. Transitions are connected to a set of input as well as a set of output places. Arcs are used to connect places and transitions. The direction of an arc shows the way tokens flow between a place to a transition or vise versa. A change in the system state occurs when a transition fires.

The firing of a transition constitutes the removal of tokens from input places and depositing tokens in the output places. Each of these objects (i.e. places, arcs and transitions) have their own sets of attributes. Objects other than the ones just discussed, which may exist on a CPN page are text blocks and local declaration pages. These are not discussed here for the sake of brevity. Mapping of individual CPN pages from the corresponding DFDs is explained in the following sections. 


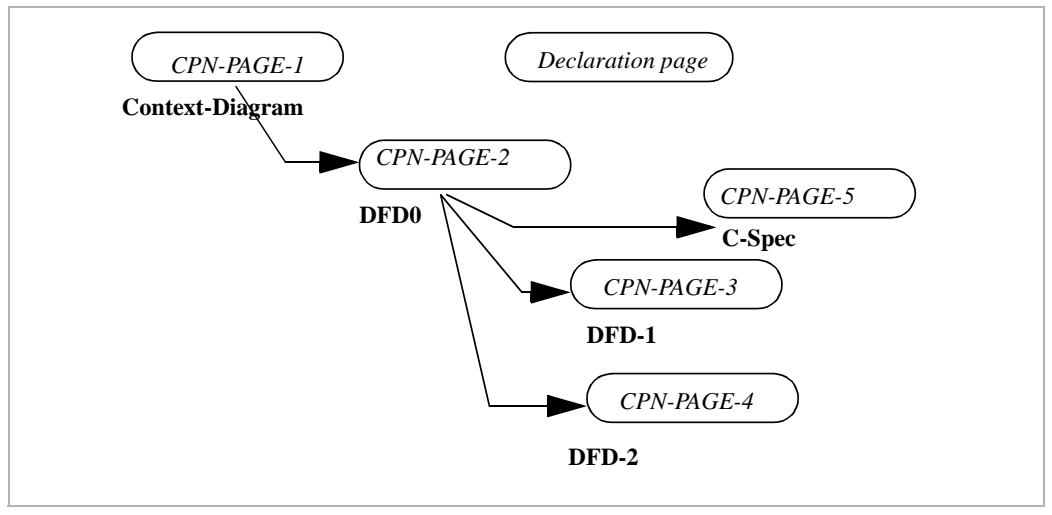

Figure 4-3 Mapping SART diagrams to CPN pages

The following sections give the representation of SART and CPN models as used in this work. The SART models are based on CDIF standard and the CPN models are described using the notation suggested by Kurt Jensen [37].

\subsection{Representation of SART models}

Based on CDIF, a representation of SART models is described in the following paragraphs. CDIF is defined by the Electronic Industries Association as a standard for CASE tool and repository communication. This standard is used for exchanging information among CASE tools and repositories. It includes descriptions, placement and details of text and graphical elements. CDIF was selected for this work because it is supported by most CASE tools vendors, including teamwork and StP.

As defined in CDIF, a SART model (MSART) is expressed as N-tuple. In this work only a 4-tuple variant of CDIF is considered, as shown below:

$\operatorname{MSART}=\left(O b j \_d f d, o b j \_d d, o b j \_s t d, o b j \_p s\right)$

Where:

- Obj_dfd: A data flow diagram object. 
- Obj_dd: The collection of Data Dictionary Entries (DDE) holding information on data flows, control flows and data stores.

- obj_std: State Transition Diagram.

- obj_ps: Object containing a P-Spec.

The hierarchy definitions are embedded in the individual objects like data flow diagrams, state transition diagrams, data dictionary entries and P-Specs.

\subsection{Representation of CPN models}

The hierarchical colored petrinets (HCPN) are defined as a tuple. S: a finite set of pages such that each page $s \in S$ is a non-hierarchical $C P N=\left(\sum_{s}, P_{s}, T_{s}, A_{s}, N_{s}, C_{s}, G_{s}, E_{s}, I_{s}\right)$. The elements for a single CPN page were described in Chapter 1. More CPN elements are described below:

- $\mathrm{SN} \subseteq \mathrm{T}$ : a set of substitution nodes. These are substitution transitions for representing a subpage in the Design/CPN hierarchy,

- SA: a page assignment function defined from $\mathrm{SN}$ into $\mathrm{S}$ such that no page is a subpage of itself,

- $\mathrm{PN} \subseteq \mathrm{P}$ : a set of port nodes,

- PT is port type function defined from PN into \{in, out, in/out, general,

- PA: port assignment function defined from SN into binary relations as:

- Socket nodes are related to port nodes.

- Socket nodes are of the correct type. 
- Related nodes have identical color sets and equivalent initialization expression.

- $\mathrm{FS} \subseteq \mathrm{P}_{\mathrm{S}}$ is a finite set of fusion sets such that members of a fusion set have identical color sets and equivalent initialization expressions.

- FT is a fusion type function defined from fusion sets into \{global, page, instance $\}$,

- $\mathrm{PP} \subseteq \mathrm{S}_{\mathrm{MS}}$ is a multiset of prime pages,

The SART objects are mapped to HCPN using the rules given in the following section.

\subsection{Mapping the SART model to a HCPN model}

These rules address the high level mapping between SART and HCPN domains. The rules address three types of SART objects: DFD, STD and DD. Each of these objects is mapped to a corresponding object in HCPN. For example, according the first rule, all the DFD objects will be mapped to non-hierarchical CPN pages.

Rule: $\forall$ obj_dfd $\rightarrow$ non-hierarchical CPN page

Rule: $\forall$ obj_std $\rightarrow$ non-hierarchical CPN page

Rule: $\forall$ obj_dd $\rightarrow$ CPN declaration page

The obj_ps (the process specifications) is not mapped automatically using a semantic transfer utility; rather the analyst converts the process specifications to a code suitable for the CPN. This code can be used in the CPN related functions and the transition code segments.

\subsection{Mapping the Data Flow Diagram to CPN page}

A data flow diagram is a six tuple DFD $=($ dfd_buble, dfd_store, dfd_term, dfd_tb, dfd_csc, dfd_flow). These are described as: 
- dfd_buble represents a process.

- dfd_store represents a data or control store.

- dfd_term represents a terminal or an element external to the system under analysis.

- dfd_tb represents a text body embedded in the DFD.

- dfd_csc represents a control specification.

- dfd_flow represents a data or control flow.

Each CPN page is a tuple object $\left(\sum, \mathrm{T}, \mathrm{P}, \mathrm{G}, \mathrm{A}\right)$. These objects are mapped from the data flow diagram object as given below:

Rule: $\forall$ primitive dfd_buble: dfd_buble $\rightarrow \mathrm{t}$ such that $\mathrm{t} \in \mathrm{T}$.

Rule: $\forall$ non-primitive dfd_bubble: dfd_buble $\rightarrow$ SN. Such that the input and output flows of this dfd_buble are mapped to the sockets. The input and output flows are mapped to ports in the subpage. In the same step, based on the flow directions on the DFD, PT is also defined from the set of its values $\{$ in, out, in/out, general $\}$.

Rule: $\forall$ dfd_store $\rightarrow \mathrm{f}(\mathrm{T}, \mathrm{P}, \mathrm{G}, \mathrm{A})$. A DFD store is represented by a set of transitions, places, guards and arcs. For a simple one input and one output store, will be represented by an input place/transition, a store place, and output place/transition set. Guards can be used to make the set read-only or read-write.

Figure 4-4 shows a simple dfd_store and its CPN map. The store has an input data flow $A_{1}$, and an output data flow $A_{2}$. The store also has a defined capacity to hold certain amount of data of type A. This store could be read-only (RO), write-only (WO) or read-write (RW). Once mapped to a CPN, the store can be represented by three places, two transitions and the connecting arcs. Guards can be used to control the flow of tokens for RO, WO, and RW implementations. 
Place attributes of time delay, the number of tokens, and initial marking can be used to map the status of the store.

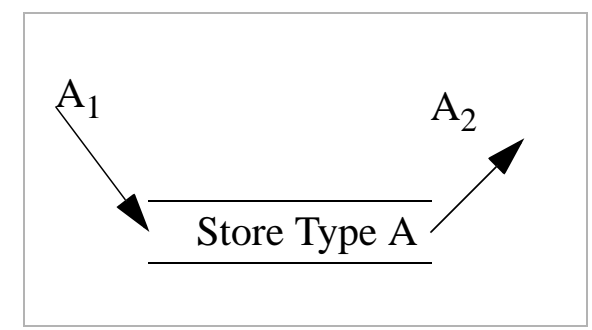

(a) A simple Store

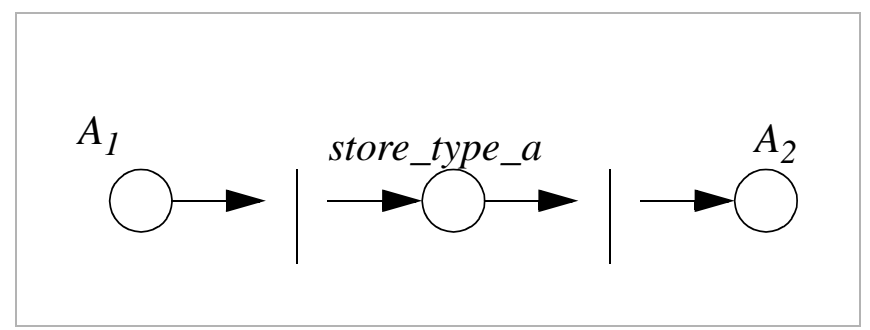

(b) CPN map

Figure 4-4 DFD Store and the CPN mapping

Other rules for the mapping a data flow diagram are:

Rule: $\forall$ dfd_term $\rightarrow \mathrm{P}$

Rule: $\forall$ dfd_csc $\rightarrow$ CPN page

Rule: $\forall$ dfd_flow $\rightarrow \mathrm{P}$

As shown in Figure 4-5, a primitive dfd_buble "Validate_Command" (Figure 4-5-a) is mapped to transition with the same name (Figure 4-5-b). The input data flows called PDB, Operator_Command_1 and Operator_Command_2 are mapped to the places with the same name. The direction of arcs in Figure 4-5-b is also mapped from the corresponding direction of data_flows shown in Figure 4-5-a. The same thing is true for the outputs of the process "Validate_Command". As shown in Figure 4-5-b, the output places spacecraft_realtime_command is an output port and PDB is an input port. In other words, these places are connected to the corresponding input/output sockets on a superpage. 
(a)
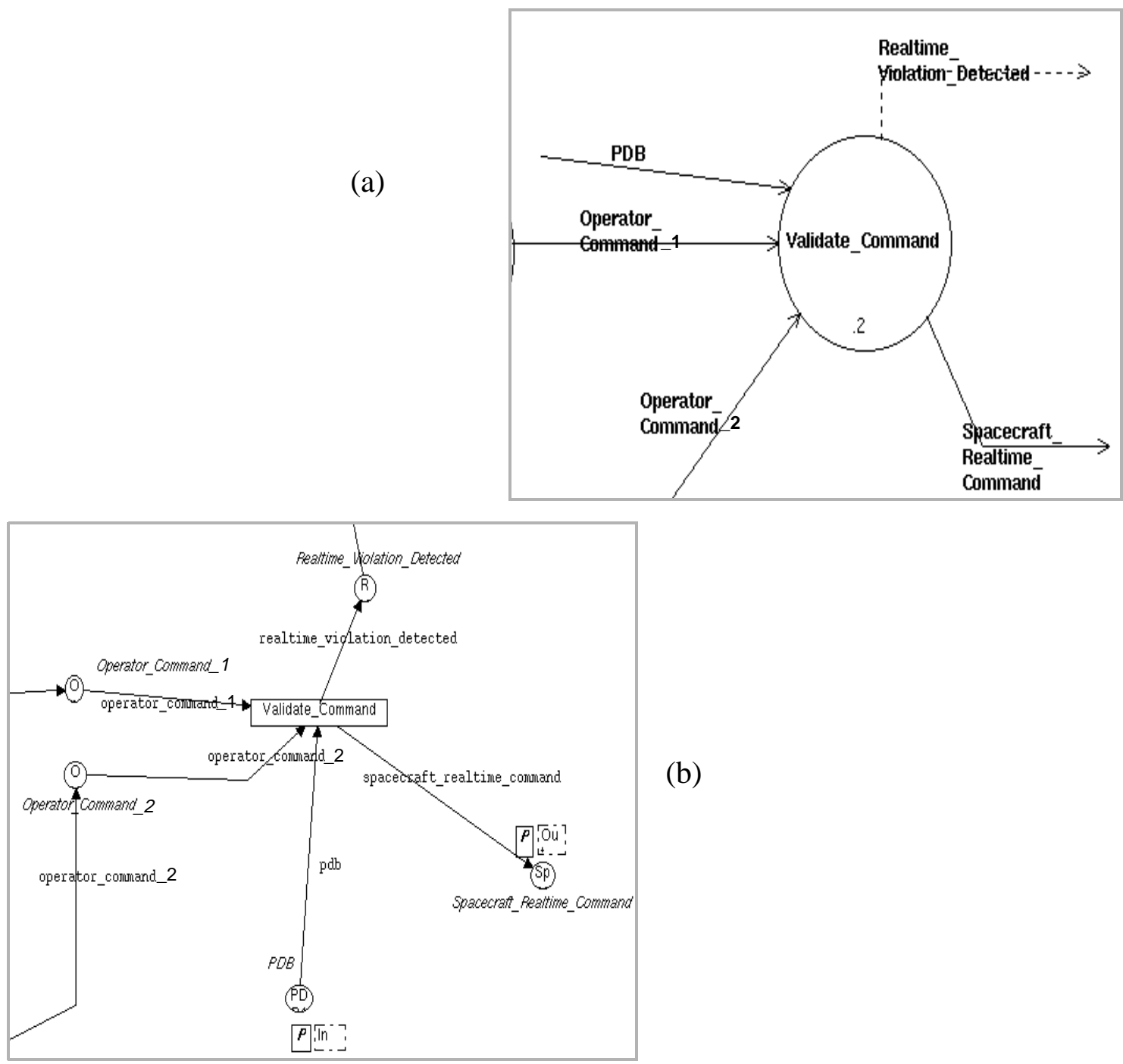

(b)

Figure 4-5 (a) SART representation of "Validate_Command", (b) The CPN representation of "Validate_Command".

\subsection{Mapping the Data Dictionary Entries}

Data dictionary entries are mapped to corresponding color entries in the global declaration page. In SART the data dictionary element is a three tuple (dde_name, dde_attr_list, edif_body). During the mapping process a dde_name is used for adding a particular color. The objects dde_attr_list (the attribute list for a DDE) and edif_body are used to generate multisets corresponding to a color set in the CPN environment. Table 4-1 shows some rules for the DDE 
mapping process.

\begin{tabular}{|c|c|c|}
\hline DDE definition & CPN translation & Remarks \\
\hline $\begin{array}{l}\text { Activity_Violation_Detected } \\
=\text { ["TRUE"| |FALSE"] }\end{array}$ & $\begin{array}{l}\text { color Activity_Violation_Detected } \\
\text { = with TRUE |FALSE; }\end{array}$ & $\begin{array}{l}\text { complete defi- } \\
\text { nition }\end{array}$ \\
\hline $\begin{array}{l}\text { ADCs-Data2 } \\
\text { = Information_Dialog } \\
\text { + Dialog } \\
\text { + Algorithms } \\
\text { + Ancillary_Data } \\
\text { + Data_Products } \\
\text { + Data_Information }\end{array}$ & $\begin{array}{l}\text { color ADCs-Data2 } \\
\text { = record Information_Dialog } \\
\text { * Dialog } \\
\text { *Algorithms } \\
\text { *Ancillary_Data } \\
\text { * Data_Products } \\
\text { * Data_Information; }\end{array}$ & $\begin{array}{l}\text { complete defi- } \\
\text { nition }\end{array}$ \\
\hline $\begin{array}{l}\text { Alarms_Notification }= \\
*_{\text {not-defined }} *\end{array}$ & $\begin{array}{l}\text { color Alarms_Notification }=\text { with } \\
\text { Alarms_Notification; }\end{array}$ & Default color \\
\hline $\begin{array}{l}\text { When the definition of Alert_signal } \\
\text { is not found in the DDE table }\end{array}$ & $\begin{array}{l}\text { color Alert_Signal }=\text { with } \\
\text { Alert_Signal }\end{array}$ & Default color \\
\hline
\end{tabular}

Table 4-1 Mapping rules for generating the Global Page of the CPN model.

\subsection{Mapping the Control Specifications}

The C-Spec in a DFD appears as a substitution transition on the corresponding CPN page. A subpage for this substitution transition represents the mapping of its C-Spec. A state transition diagram is the tuple (std_state, std_tb, std_trans):

- std_state is the object representing state in the STD,

- $\quad$ std_tb is used to give a definition to an STD as Mealy or Moore,

- $\quad$ std_trans represents the transition object,

The mapping rules used are:

Rule: $\forall$ std_state $\rightarrow \mathrm{P}$

Rule: $\forall$ std_trans $\rightarrow \mathrm{T}$ 
Rule: $\forall$ std_tb $\rightarrow \mathrm{f}(\mathrm{G}, \mathrm{C}, \mathrm{P})$ where both guard (G) as well as code segments $(\mathrm{C})$ are derived from the conditions needed for the change in the system state. The places $(\mathrm{P})$ correspond to the input and output signals.

A cruise control example is presented in Figure 4-6 to illustrate the mapping process for STDs.

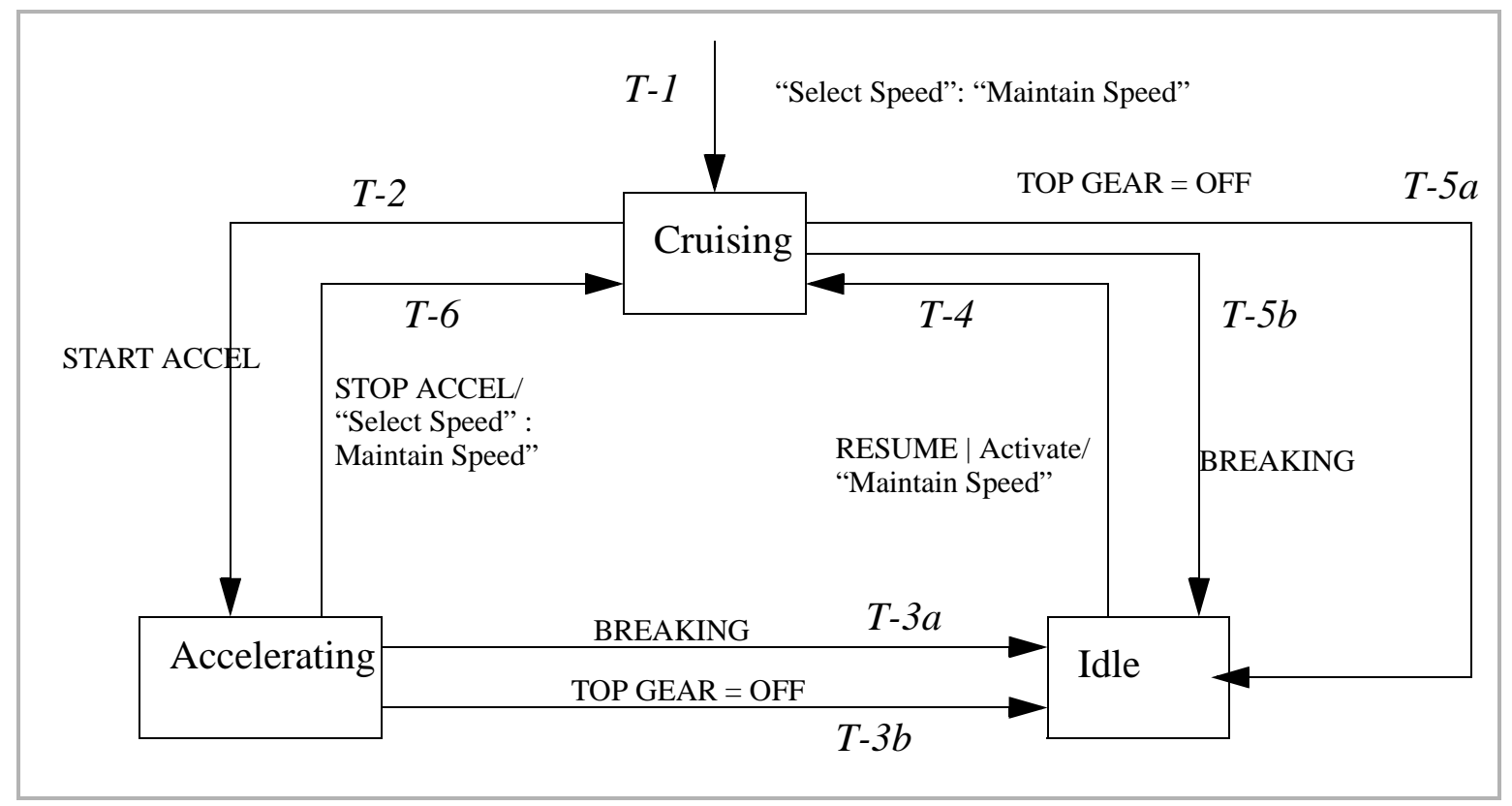

Figure 4-6 Cruise Control STD.

The transitions are marked as T-1 through T-6 for a comparison with transitions in the CPN page shown in Figure 4-7. The set of transitions T-3a,b is shown as two separate transitions for simplicity sake. The same is true for T-5a,b. Both of these can be represented by one transition as well. The places are named according to the mapping rules given earlier. Two places named as Enable_Select_Speed and Enable_Maintain_Speed are used for enabling/disabling the processes, Select_Speed and Maintain_Speed, respectively. The presence or absence of a token in these places is used to enable or disable the corresponding process.

Different shades are used to distinguish between places in Figure 4-7. The places shaded 
black are the enabling/disabling places. The larger size places shaded grey are the states of the controller. The places with a light grey shade are input signals to the controller. There are always tokens present in the input places. For example, the place Braking has a token with a value TRUE if the brakes are on, otherwise it is false. Guards for the transitions are shown in a separate labeled box to reduce diagram clutter. Blank spaces in the STD names are replaced with an "under score" character. The CPN diagram contains enable_* place for every enabling action in the STD, e.g., "Select Speed" will be mapped to "Enable_Select_Speed".

Note: Arc inscriptions and intermediate place names in Figure 4-7 are hidden from the view for the sake of clarity.

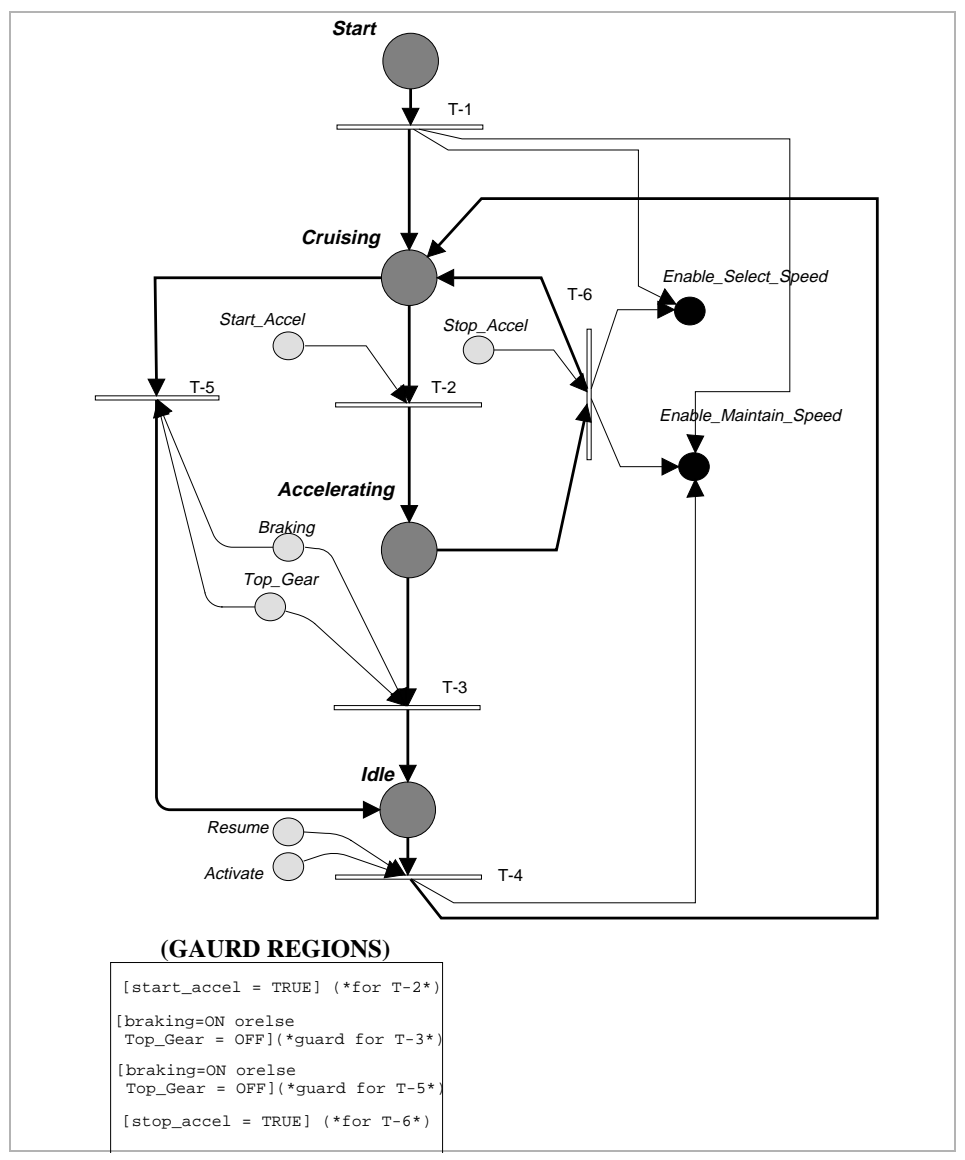

Figure 4-7 CPN diagram for Cruise 
The important aspect related to the enable and disable function of an STD is based on the Hatley and Pirbhai notation. Actions (enabling and disabling) are associated with transitions that are transient in nature. The actions are assumed to continue in effect until the next transition occurs. This means a process activated by a particular action remains activated continuously and continues to respond to changing data inputs until the next transition occurs [38]. The approach used in [38] is slightly different. The enabling places get one token in the state where a process is to be enabled. Once the token is consumed by the enabled process, there is no more enabling token until the system goes back to the same state. The Hatley and Pirbhai approach can be incorporated through slight modifications to our mapping rules.

\subsection{Parameterization and Simulation}

The mapping rules described previously in the last four sections are used for generating primitive models (Step 4 of Figure 4-1). These primitive models contain the semantics of CPN pages (hierarchically arranged), places and transitions on the individual pages and color definition of the tokens. In Step 5 of the proposed process (Figure 4-1), the IV\&V analyst adds to these models, more details such as timing distribution, initial marking, and guard conditions etc.

Parameterization step can be further explained using the example of cruise control. For this example, Figure 4-6 and Figure 4-7 show the STD and CPN diagrams respectively. From the STD in Figure 4-6, we see that the system will go from "Cruising" state to "Accelerating" state when "START ACCEL" is TRUE. This move is modeled by the transition T-2 in Figure 4-7. The analyst will edit its guard condition as "Start_Accel = True". Timing information can also be added to T-2 for modeling the associated delay when the system goes from "Cruising" to "Accelerating". For the place "Start", the IV\&V analyst will add an initial marking to ensure that T-1 will fire as soon as CPN page of Figure 4-7 becomes active. Similar information is added to or edited for other nodes in the diagram by the IV \&V analyst during this step.

In Step 6 of the proposed process, simulation tool of Design/CPN is used to analyze the 
behavior of the system. Simulation reports are generated and further analyzed for timing deadlocks, reachability analysis and resource usage etc. Other uses of the simulation based analysis conducted using the proposed process, include: Performability analysis [43], Risk assessment [44], [55] and [56], and Identification of high risk scenarios [45]. Chapter 6 gives more detail about simulating a component of a large software system.

\subsection{Tracking upgrades in Developer's models}

Software systems evolve from very high level requirements supplied by the customer (Figure 1-1). These requirements are utilized for generating detailed requirement specifications, design, and implementation. During the software system's development process, the requirements may go through substantial changes. This results in upgrades of the developer's models being supplied to the IV\&V team.

The framework proposed in this work, addresses the changes in SART models depending on the type of a given change in the developer's models. Different types of changes that may occur in an SART model are:

- Addition or removal of a bubble or a flow (data or control),

- Change in the data dictionary,

- Addition or removal of a DFD or a C-Spec,

- Combining two SART models

A bubble is represented by a transition in the CPN model. If a new bubble is added in the SART model, a transition with a set of input/output places will be added to the corresponding CPN page. The reverse is true if a bubble is removed. A flow is represented by a set of arcs, a place, and a color definition in CPN. When a flow is removed, the corresponding arcs, place and color definition are removed. A change in the data dictionary will result in the change of color 
definitions in the declaration page CPN.

An addition of a DFD/C-spec will result in a new CPN page. This page can be added to the existing model by using the substitution transition. The mapping utility will generate primitive CPN page from the new DFD/C-spec. The IV\&V analyst will manually add this page to the existing Design/CPN model and upgrade the declaration page. On the other hand when a diagram is removed, the corresponding CPN page and the substitution transition is manually removed.

If two SART models are combined together by the developer, then the corresponding CPN models can also be joined using substitution transitions. The declaration pages are combined as well. This step is manually performed by the IV\&V analyst. This technique was used in this work when different models of Chapter 6 were combined together to form larger models.

\subsection{Summary}

This chapter provided a framework for generating dynamic models from SART. Using this framework, a large software system was analyzed as described in Chapter 6. The framework and the SART related dynamic analysis is the first contribution (Chapter 1) of this work. The following chapter presents the second contribution of this work related to the framework for generating dynamic models for UML models based on DMM. 


\section{Chapter 5 A Framework for Dynamic Analysis of UML}

The previous chapter described first contribution of this work related to the framework for generating dynamic models from SART models. This chapter presents the second contribution of this work related to generating dynamic models from UML models. UML is the successor to the wave of OO notations (Booch, OMT, and Jacobson) and it is expected to be the standard modeling language in the future (Martin Fowler [39] ). This chapter lays the framework for the dynamic analysis of the UML. A high level view of this approach is shown in Figure 5-1. In stage-1 the semantics of UML models are converted in DMM notation. This is followed by the stage- 2 where semantics information contained in DMM is mapped to an environment suitable for performing dynamic simulations. In this work, Design/CPN provides such environment. Besides CPN other environments like ROOM (realtime object oriented modeling) can also be used for performing dynamic simulations. More detailed steps of the proposed framework are described in Section 5.2 .

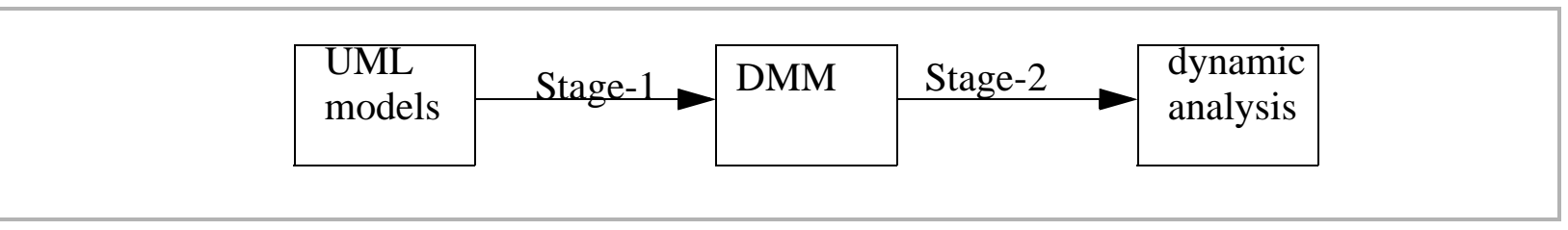

Figure 5-1 Framework for using DMM models for dynamic analysis of software specifications

A brief introduction of UML notation was given in Chapter 1. UML consists of six different diagrams. Out of these, four diagrams address the elements of system's logical model. These four can help model dynamic behavior of the modeled software system at the early phases of development. Therefore the proposed DMMs will be developed using the four diagrams of the logical model. In this chapter, semantics of the logical model will be described. The components of the four diagrams containing the information regarding the dynamic behavior of the system 
will be separated. These components will be expressed in terms of a set of Dynamic Metamodel component. The last section will show the rules to map the dynamic metamodel components to a CPN model.

The following sections present a DMM mapped from a sample UML model of a pacemaker. Earlier work on DMM elements is given in [40]. DMM is an environment proposed in this work to capture syntax and semantics related to dynamic behavior of a given software system. The technique draws upon substantial work by Nissen and Jarke [27] that uses metamodels to develop requirement documents for large software systems. DMM on the other hand is suggested to be used in the dynamic analysis of the requirement models. Thus a DMM is a class of metamodels, that can capture dynamic behavior from a specification model. The DMM is then utilized to build a CPN model.

DMM can be beneficial due to two reasons. First is that DMM can help deal with multiple views and models within UML. Secondly, DMM can be extended further to capture dynamic behavior from other $\mathrm{OO}$ methods. The first step towards building a DMM is to analyze the UML models for their dynamic characteristics. The second step is to identify basic elements of DMM and define their associations.

An important aspect that may not be tackled at this phase of research is developing translating utilities based on DMM. The reason being the absence of explicit format to interchange UML models. Even for version 1.1, OMG (Object Management Group) has just issued an RFP (Request for Proposal) for SMIF (Stream based Model Interchange Format) [47] to allow interchange of UML models. Considering the recent issuance of the RFP, it may take a while before SMIF gets standardized. Whenever SMIF or XML (Extensible Markup Language) become a standard, either one will help to develop translating utilities based on the DMM.

An overview of DMM elements is given in Section 5.1. Mapping rules are given in Section 5.2. An example of a pacemaker in UML and its translation to DMM is given in Section 
5.3. DMM can be used to generate models suitable for CPN simulation tools, as addressed in Section 5.4.

\subsection{DMM (Dynamic Metamodel)}

DMM is a class of metamodels that can be used to capture dynamic behavior across different notations of software system models. While requirements' models are abstract representations of an existing or desired real world, metamodels are abstract representations of an existing or desired requirement models and their interrelationship [27]. DMM further extends the concepts of Jarke's metamodels by combining it with RLP (Requirements Language Processor), Davis [51].

Davis first describes SREM (Software Requirements Engineering Methodology) to be used for requirements analysis. Using SREM [50], a tool set REVS (Requirements Engineering Validation System) was developed. Both the tool set and the methodology are based on a notation called R-Nets, an extension to conventional FSM. Davis points out that monolithic FSM seemed to be too unwieldy for the requirements specification of complex real time systems. Davis then describes another system called RLP to be better than SREM. The reason is that RLP uses the stimulus-response sequence, a trace of a two-way dialog between the system under specification and its environment. Davis suggests the sequence selection should be based on typical dialogues.

Some of the problems with FSM have been addressed by the current version of statecharts [46]. Statecharts allow nesting of state, transitions to and from the superstates, orthogonal or concurrent state, provision of history in a substate and a provision of Mealy and Moore in the same environment. Even though problems such as the state explosion still exist, FSM is widely used in the software systems community. Therefore DMMs rely on statecharts for some of the semantics while using stimulus-response sequence [51] to build execution paths for dynamic behavior. 
For dynamic analysis of UML, a scenario will be chosen from the Use-Case diagram. Inputs for that scenario will act as stimulus for an execution path. An execution path will be developed using interaction diagrams. Each execution path contains a series of activities. The activities will model the operations performed by an object during its lifetime. Interaction of activities from different execution paths will depend on the associations between corresponding classes (represented in class diagrams) and collaboration of objects (represented in interaction diagrams).

The characteristics needed for a DMM include the representation of objects and associated methods, the association among objects, the dynamic behavior of a given object, and the data and control flow.

\subsubsection{Metamodels as Applied to Software Development}

UML consists of several models. Each of these models encompasses a specific aspect of the dynamic behavior. There is a need to create an environment to help gather information related to dynamic behavior from different models. There are few examples in the literature where metamodeling has been used in the software development process. One example [27] shows the application of metamodels in the very beginning of a software development lifecycle, i.e., elicitation of requirements. However it does not address the problems faced in the subsequent phase of requirement analysis. This work builds on the metamodeling approach suggested in [27]. Three of the important aspects related to metamodels are described in the following subsections.

\subsubsection{Using Metamodels to address multiple perspectives}

In a large software system, the first challenge is to lay down the requirements. In the second phase these requirements need to be analyzed for their soundness of static and dynamic properties. A good metamodel should:

- contain a small number of easy-to-understand basic constructs (elements and related 
attributes and functions) so it can serve as a basis for human communication,

- cover all the individual methods used in the given methodology.

The application of metamodeling to the first phase, i.e., requirement elicitation, does provide us the opportunity to see how metamodels can help combine information from different perspectives. When software systems are being planned, the customer decides to set up an internal team that can put together its requirements. A developer team is asked to interact with the customer team to further refine the requirements. This presents a dilemma of different groups using different models to present their view of the software systems under development.

The method of metamodeling presented in [27] is used to gather requirements from customer and the development teams. This method, called PFR (analysis of Present and Future Requirements) combines source and business perspectives. A source perspective captures the interaction between different entities as well as the scenarios critical to the operational success. Business perspectives capture the usage profile of the system to be developed. There are three main steps involved in this process of developing source and business perspectives.

In the first step, a goal structure is defined based on the current business processes. In the second step the perspectives identified as critical are captured by workflow analyses. The acquisition process is accompanied by a cross-perspective analysis of the captured information for consistency and completeness. In the third step the requirements document is developed. The goal is to draw together individual perspectives. This is where metamodels are used to gather information from different perspectives. The step is accompanied and followed by the development of a comprehensive requirements document of typically several hundred pages. When different perspectives are merged, the key issues are integration of syntax and semantics.

\subsubsection{Integrating syntax using Metamodel}

In developing a model, different perspectives reflect the same software systems. 
Therefore, the modeling constructs should reflect a shared understanding of what concepts are likely to be interesting to model. As in any modeling activity, choosing what aspects of models to abstract depends on the purpose of the metamodel and the perceived obstacles to achieving this purpose. Syntax integration for UML models is based on two of the above perspectives:

- information-exchange,

- $\quad$ activity-sequence.

Similar to the business perspective in [27], use-cases become the basis for providing scenarios necessary for generating these perspectives. Sequence diagrams further elaborate the series of activities in the activity-sequence perspective. Statecharts provide a sequence of activities in the activity-sequence as well as information-exchange perspectives.

\subsubsection{Integrating semantics using Query classes}

It is suggested in [27] that query classes can be used to define consistency of knowledge within an individual perspective as well as the consistency between different perspectives. The development of these queries begins during construction of a metamodel structure and continues throughout the projects. For integrating semantics of UML models, a set of core query classes is proposed. More queries will be built around this core, based on the details of dynamic analysis and domain specific analysis.

The query classes consist of queries for:

- generating dictionary containing global level and local level data declarations,

- interpreting textual annotations from different UML diagrams,

- generating timing distribution for activity-sequence perspective,

- generating sequence of activation, 
- gathering the list of external inputs and outputs and related timing information.

The following subsection summarizes the characteristics of a DMM.

\subsubsection{Characteristics of a DMM}

As described earlier in the section on UML, a given software system can be represented by a number of models. These models may capture a given aspect of the dynamic behavior of the modeled system. In the case of UML, the class structure contains the functions processing a set of data. The associations show the interaction among different objects. Statecharts contain the sequence of activation of different functions. The interaction diagrams provide the timing and probability distribution for different functions.

DMMs provide a structure for capturing dynamic behavior of a software system. Therefore they need to allow features to process the available information about a software system to perform the following tasks such as to find the bottlenecks in the system, help find any timing information that may be needed by the analyst, provide ways to assess resource usage, conduct performability analysis, and perform dynamic complexity and severity analysis.

These DMM goals can be met if there are ways to extract the following aspects of UML:

- model the methods in an object,

- model the associations among different objects,

- model the type and value of different units of data flow,

- model the sequence of activation of methods within an object,

- capture the timing and probability distribution from the interaction diagrams.

A stimulus response [51] paradigm is the core of DMM. UML's interaction diagram can 
provide the information for all the external stimuli and the corresponding responses. For individual stimulus, a series of actions is also defined in the interaction diagram. For each action, a set of objects collaborate to perform a role. In each role the participating objects provide a set of methods as defined while developing the class diagram. These methods follow an activation path as described in the statecharts related to the individual classes. Figure 5-2 elaborates the stimulus response point of view.

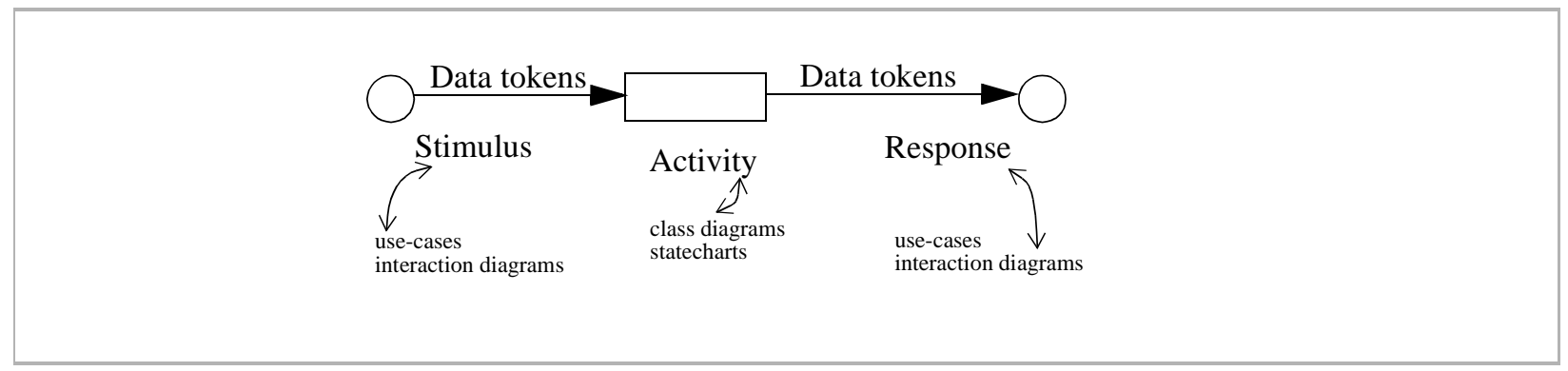

Figure 5-2 UML diagrams and Stimulus Response paradigm

Both stimulus and response are in the form of data tokens entering and leaving the system, respectively. A DMM element mimicking a container or a bucket (represented by a circle) will be used for holding the data tokens. A path for the flow of these tokens determines the flow direction. The flow of tokens will be modeled by a relation (represented by an arrow). An activity (represented by a rectangle), shows the entity responsible for converting the input data tokens (stimulus) to the output data tokens (responses).

Figure 5-2 also shows a stimulus for a particular scenario will come from Use-Case and interaction diagrams. The activity will be modeled on the semantics of the operations and associations defined in the class diagrams. The sequence defining how different activities will be on or off comes from statecharts.

The view of dynamic behavior given in Figure 5-2 is rather simplistic in a way that a large software systems may have lot more activities spread in different levels of its definition. DMM also provides a way for hierarchical representation of different levels of systems description. This 
will be achieved with the use of parent and child levels. Special DMM elements to link two levels are required, as shown in Figure 5-3, where $A_{m}-1$ represents all the activity on the child level.

Keeping these characteristics in mind, DMM elements are listed in the following section. These elements are based on the experience gained from CPN based analysis of realtime systems (Chapter 4 and Chapter 6).

\subsubsection{Elements of a DMM}

In reference to the stimulus response paradigm, a two level DMM is shown in Figure 5-3. The DMM elements used in the Figure 5-3 are: $D_{m^{-}}-1 ; \mathrm{B}_{m^{-}}-1,2,3 ; \mathrm{cb}_{\mathrm{p}^{-}}-1,2 ; \mathrm{cb}_{\mathrm{c}}-1,2 ; a_{m}-1,2,3,4 ; A_{m^{-}}$ $1 ; G_{m}-1 ; F_{m}-1$; and $E_{m}-1,2$. The meanings of these symbols are described later. It may be beneficial to look at the flow of data tokens first, as explained in the following paragraphs.

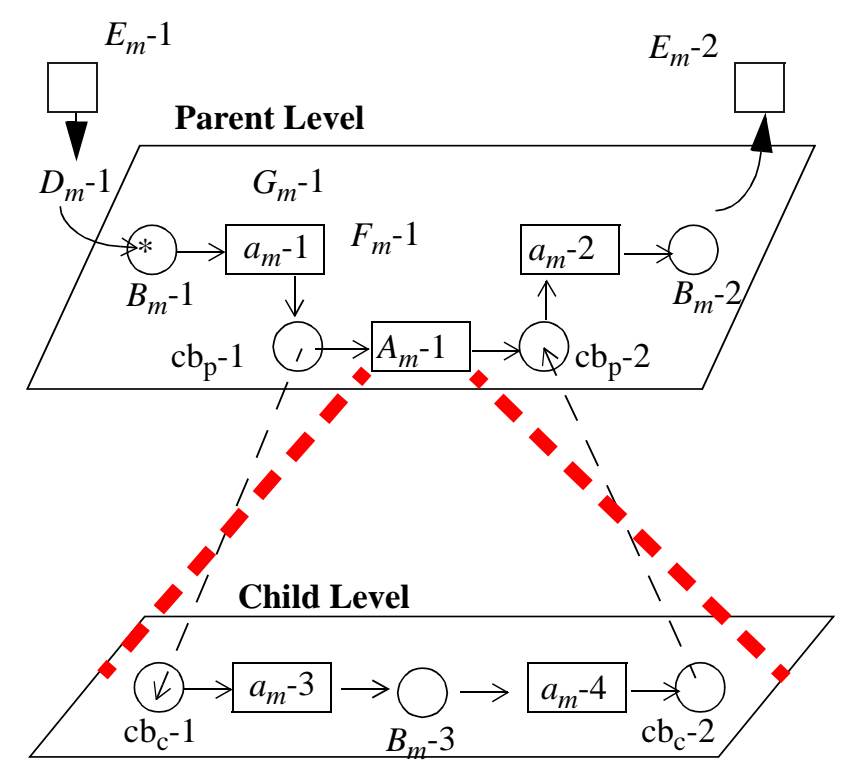

Figure 5-3 Different elements of a DMM

Dynamic behavior modeled by DMM of Figure 5-3 can be described as a series of activities. A Meta-agent $\left(E_{m}-1\right)$ supplies the system with a Meta-stimulus in the form of a data 
token $D_{m}-1$. At the start of simulation, the data token $D_{m}-1$ (in the parent level), is residing in $B_{m^{-}}$ 1 , where $B_{m}-1$ acts as a container or a bucket for the data token. Once it is processed by activity $a_{m}-1$, the data moves to $\mathrm{cb}_{\mathrm{p}}-1$. From $\mathrm{cb}_{\mathrm{p}^{-1}}$ it gets processed by $A_{m}-1$ (the child level). Therefore the data moves from $\mathrm{cb}_{\mathrm{p}}-1$ to $\mathrm{cb}_{\mathrm{c}}-1$. Here it gets processed by $a_{m}-3$ and deposited in $B_{m}-3$. When the data is processed by the next processor $a_{m}-4$, it moves from $\mathrm{cb}_{\mathrm{c}}-2$, to $\mathrm{cb}_{\mathrm{p}}-2$. The next processor $\left(a_{m}-2\right)$ deposits the token in $B_{m}-2$.

$s_{m}$ for the whole system is the number and type of data tokens present in $B_{m}-1$ at the beginning of execution (i.e., $D_{m}-1$ in this example). $r_{m}$ for the whole system is the number and type of tokens present in $B_{m}-2$ at the end of execution. $s_{m}$ for the individual activity is the number and type of data tokens present at its input before the execution. $r_{m}$ for the individual activity is the number and type of tokens present at its output after its execution.

The activity $a_{m}-1$ shows two labels $G_{m}-1$ and $F_{m}-1$. The first label $G_{m}-1$ represents an enabling condition or a guard for the activity. A data token present at the input of activity $a_{m}-1$ will not be processed until $G_{m}-1$ is true. Once $G_{m}-1$ is true, activity $a_{m}-1$ turns on and processes the token as well as executes the associated function $F_{m}-1 . F_{m}-1$ may contain a set of processes to be executed whenever activity $a_{m}-1$ is on.

The preceding paragraphs contain the basic elements of a DMM. To summarize, a DMM is a 12-tuple:

$$
\left(D_{m}, s_{m}, r_{m}, B_{m}, b_{m}, c b_{m}, a_{m}, A_{m}, G_{m}, E_{m}, R_{m}, F_{m}\right)
$$




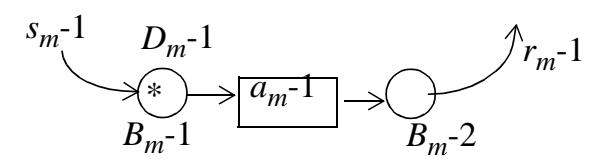

Figure 5-4 Example of Meta-data, Meta-Stimulus, Meta-response, Meta-bucket

These elements are summarized in Table 5-1. Further detail of the DMM elements is given in Section 5.1.3.1 through Section 5.1.3.12.

\begin{tabular}{|c|c|}
\hline Elements & Description \\
\hline $\begin{array}{l}\text { Meta-data } \\
\left(D_{m}\right)\end{array}$ & $\begin{array}{l}\text { Set of information tokens flowing between different elements of a DMM. Its attributes are the } \\
\text { value and type. }\end{array}$ \\
\hline $\begin{array}{l}\text { Meta-stimulus } \\
\left(s_{m}\right)\end{array}$ & $\begin{array}{l}\text { Type of Meta-data that may trigger an event. Meta-stimulus can either come from an external } \\
\text { (e.g., Meta-agent) or a local source (e.g. Meta-activity). Its attributes are the same as Meta-Data }\end{array}$ \\
\hline $\begin{array}{l}\text { Meta-response } \\
\left(r_{m}\right)\end{array}$ & $\begin{array}{l}\text { Type of Meta-data generated by a Meta-activity. This can in turn be used by another Meta-activ- } \\
\text { ity as a Meta-stimulus, or it can be sent to a Meta-agent. Its attributes are the same as Meta-data }\end{array}$ \\
\hline $\begin{array}{l}\text { Meta-bucket } \\
\left(B_{m}\right)\end{array}$ & $\begin{array}{l}\text { Specifies a container where Meta-data can stay while waiting to be processed by the next Meta- } \\
\text { activities. Its attributes are type and number of units of Meta-Data it contains at a given time. } \\
\text { Another attribute is the length of time Meta-data has to wait in the bucket before moving on to a } \\
\text { Meta-activity. }\end{array}$ \\
\hline $\begin{array}{l}\text { Enabling- } \\
\text { bucket }\left(b_{m}\right)\end{array}$ & A subtype of Meta-bucket, it is used for modeling the enabling function present in a statechart \\
\hline $\begin{array}{l}\text { Connection- } \\
\text { bucket }\left(\mathbf{c b}_{m}\right)\end{array}$ & $\begin{array}{l}\text { There are two kinds of connection buckets, parent }\left(\mathrm{cb}_{\mathrm{p}-m}\right) \text {, and child }\left(\mathrm{cb}_{\mathrm{c}-m}\right) \text {. Connection buck- } \\
\text { ets, with subscripts } \mathrm{p} \text { and c represent parent and child respectively. }\end{array}$ \\
\hline $\begin{array}{l}\text { Meta-activity } \\
\left(a_{m}\right)\end{array}$ & $\begin{array}{l}\text { An atomic action taking some information or data as input and generating new data as output. The } \\
\text { attributes are type, meta-guard, the time delay, the probability of producing a meta-response and } \\
\text { a flag indicating if a meta-function ( Section 5.1.3.12) is present. }\end{array}$ \\
\hline $\begin{array}{l}\text { Meta-action } \\
\left(A_{m}\right)\end{array}$ & An abstraction used in the parent level for representing a child level. \\
\hline $\begin{array}{l}\text { Meta-Guard } \\
\left(G_{m}\right)\end{array}$ & $\begin{array}{l}\text { A condition that must be true before a Meta-action, a Meta-activity or a Meta-relation can take } \\
\text { place. }\end{array}$ \\
\hline $\begin{array}{l}\text { Meta-agent } \\
\left(E_{m}\right)\end{array}$ & The (External to the system) source of Meta-stimulus or sink for a Meta-response. \\
\hline
\end{tabular}

\section{Table 5-1 Elements of DMM}




\begin{tabular}{|l|l|}
\hline \multicolumn{1}{|c|}{ Elements } & \multicolumn{1}{c|}{ Description } \\
\hline \hline $\begin{array}{l}\text { Meta-relation } \\
\left(\boldsymbol{R}_{\boldsymbol{m}}\right)\end{array}$ & $\begin{array}{l}\text { Specifies the association or path between a Meta-bucket and a Meta-activity. Its attributes are } \\
\text { the type of data it can handle, the time delay and the probability of letting a given Meta-data go } \\
\text { through. }\end{array}$ \\
\hline $\begin{array}{l}\text { Meta-function } \\
\left(\boldsymbol{F}_{\boldsymbol{m}}\right)\end{array}$ & The set of functions that may be executed when the system goes through a Meta-activity. \\
\hline
\end{tabular}

\section{Table 5-1 Elements of DMM}

\subsubsection{Meta-data $\left(D_{m}\right)$}

Meta-data is the set of information tokens flowing between different elements of a DMM. Its attributes are the value and type. Individual meta-tokens are members of the data dictionary for a DMM. This data dictionary is generated using the queries from Section 5.1.1.3. The data dictionary contains all the $D_{m}$ elements present either in the global or local declaration areas. Depending upon the scope of a data token, its declaration will be shown in either the global or local level of the DMM hierarchy. The declarations are mapped from attribute definitions of the class diagram (Figure 5-8). The mapping process is built around the semantic queries as mentioned in Section 5.1.1.3. An example of a meta-data is $D_{m}-1$ as shown in Figure 5-4.

\subsubsection{Meta-stimulus $\left(s_{m}\right)$}

Meta-stimulus is the type of Meta-data that may trigger an event. Meta-stimulus can either come from an external (e.g., Meta-agent) or a local source (e.g., Meta-activity). Its attributes are the same as Meta-Data. In addition, it also has an initial set of values acquired when the system is initialized. Figure 5-4 shows an example of Meta-stimulus $s_{m}-1$. Meta-stimulus are extracted from use-case diagram. This element is also present in sequence diagram (Figure 5-12) in the form of messages from actors to a system. 


\subsubsection{Meta-response $\left(r_{m}\right)$}

Meta-response is the type of Meta-data that is generated by a Meta-activity. This can in turn be used by another Meta-activity as a Meta-stimulus, or it can be sent to a Meta-agent. Its attributes are the same as Meta-data. $r_{m}-1$ in Figure 5-4 is an example of Meta-response. Similar to Meta-stimulus, an external Meta-response can be mapped from the use-case and sequence diagrams.

\subsubsection{Meta-bucket $\left(B_{m}\right)$}

Meta-bucket specifies a container where Meta-data can stay while waiting to be processed by the next Meta-activities. Its attributes are type and number of units of Meta-Data it contains at a given time. Another attribute is the length of time Meta-data has to wait in the bucket before moving to a Meta-activity. Figure 5-4 shows $\mathrm{B}_{m}-1$ and $\mathrm{B}_{m}-2$ as two meta-buckets. Presence of a Meta-data token (in $\mathrm{B}_{m}-1$ at present) determines the state of the system modeled by DMM.

\subsubsection{Enabling-bucket $\left(b_{m}\right)$}

Enabling-bucket is a subtype of Meta-bucket. It is used for modeling the enabling function present in a statechart. This bucket will result when a statechart is mapped to DMM. For example, the statechart shown in Figure 5-11, in superstate "Self_Triggered", the event ChamberSense invokes the Enable_Pace_Electronics. This behavior is captured in DMM by a $b_{\mathrm{m}}$ that is deposited with a data-token every time the event ChamberSense is present.

Enabling-bucket has the same attributes as a Meta-bucket. An additional feature of this element is a capability to purge all the remaining Meta-data present in it once the corresponding object (in UML) destroys itself. 


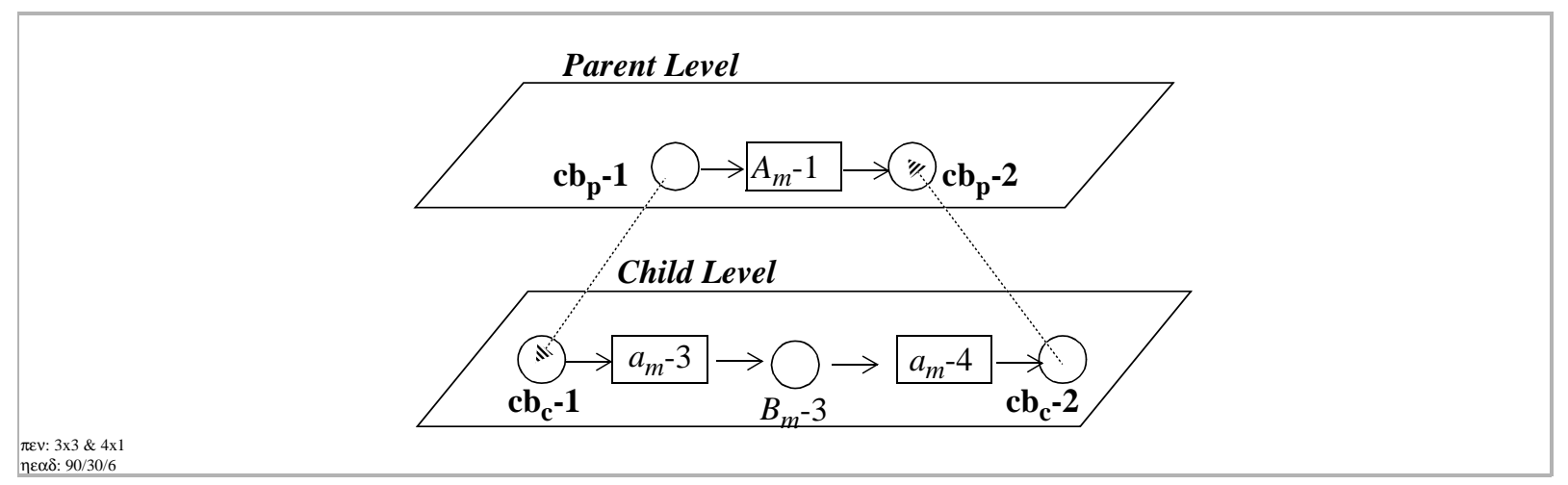

Figure 5-5 Child and Parent connection buckets

\subsubsection{Connection-bucket $\left(\mathrm{cb}_{m}\right)$}

There are two kinds of connection buckets, parent $\left(\mathrm{cb}_{\mathrm{p}-m}\right)$, and child $\left(\mathrm{cb}_{\mathrm{c}-m}\right)$, with subscripts $\mathrm{p}$ and $\mathrm{c}$ representing parent and child respectively. The subscript $\mathrm{m}$ is for a meta element. Connection-bucket is a subtype of Meta-bucket and is used to represent semantics of hierarchy in DMM.

DMM is arranged as levels in the modeling hierarchy. The upper level is called parent level and the lower level is the child level. To represent the flow of data tokens between child and parent levels, Connection-buckets are used (as shown in Figure 5-3). The names of these connection-buckets are taken from their respective levels. Thus the Connection-buckets in the parent level are parent connection-buckets and child levels contain child connection-buckets.

A Connection-bucket inherits all the attributes of the Meta-bucket. In addition it has an argument IN or OUT. For a child Connection-bucket, IN contains the Meta-data flowing from the parent system and OUT contains the Meta-data flowing to the parent system. For parent connection-bucket $\left(\mathrm{cb}_{\mathrm{p}-m}\right)$, IN and OUT represent data-tokens flowing in the opposite direction.

\subsubsection{Meta-activity $\left(a_{m}\right)$}

Meta-activity is an atomic action taking some information or Meta-data as input and 
generates new Meta-data as output. This element is mapped from the class diagram (Table 5-3) where an operation such as Coil_driver-->decode() maps to an activity decode.

The attributes are type, meta-guard, the time delay, the probability of producing a metaresponse and a flag indicating if a meta-function ( Section 5.1.3.12) is present.

The type attribute reflects the type of Meta-data that can be processed by the Metaactivity. Meta-guard is mapped from the statechart (Figure 5-11). Time delays associated with an $a_{m}$ are available from a sequence diagram (Figure 5-12). An example for the timing information is the timeout of value REFRACTORY_TIME.

\subsubsection{Meta-action $\left(A_{m}\right)$}

Meta-action is an abstraction used in the parent level for representing a child level. An example of Meta-action $\left(A_{m}-1\right)$ is shown in Figure 5-5. Present at the parent level, it behaves like a Meta-activity with a set of input and output buckets. The real behavior of this abstraction is determined by the DMM level represented as a child. Therefore the buckets of the meta-action are parent connection buckets. These parent buckets are linked to the child connection buckets as shown in Figure 5-5.

The behavior of $A_{m}-1$ is represented by the activities $a_{\mathrm{m}}-3, a_{\mathrm{m}}-4$ and bucket $B_{\mathrm{m}}-3$. For keeping DMM as simple and predictable as possible, Meta-action should not have guards (or other attributes associated with Meta-activity). Meta-stimulus from the parent level is sent to the child level and the generated Meta-response is sent back to the parent level. The dotted arrows between connection buckets show the link is for demonstration only.

\subsubsection{Meta-guard $\left(G_{m}\right)$}

Meta-guard is a condition that must be true before a Meta-activity or a Meta-relation can take place. A meta-guard is mapped from an event present in state diagram (Figure 5-11) for a 
particular state transition. An example is the event ChamberSense in the superstate Self_Triggered that invokes the operation enable_pace_electronics. The activity mapped from enable_pace_electronics will have a guard mapped from ChamberSense that must be true for the activity to process Meta-data from the input bucket and deposit it into an output bucket.

In summary, Meta-guard is a Boolean expression (that must be true for the Meta-activity to occur). The Boolean expression depends on system aspects such as type and value of data to be processed, the timing restraints and probability distribution for a given Meta-activity.

Analyst can also attach probability distribution and the meta-function to any activity. If the meta-function is present, the corresponding flag will be set.

\subsubsection{Meta-agent $\left(E_{m}\right)$}

Meta-agent is the (External to the system) source of Meta-stimulus or sink for a Metaresponse. Meta-agents are mapped from the actors present in the sequence diagram (Figure 5-12).

\subsubsection{Meta-relation $\left(R_{m}\right)$}

Meta-relation specifies the association or a path between a Meta-bucket and a Metaactivity. Its attributes are the type of data it can handle, the time delay and the probability of letting a given Meta-data go through.

\subsubsection{Meta-function $\left(F_{m}\right)$}

Meta-function is the set of functions that may be executed when the system goes through a Meta-activity.

\subsection{Mapping UML to DMM}

The elements of UML and DMM have been described in previous sections. This section 
describes the UML to DMM mapping.

The mapping of UML elements to DMM elements is an iterative process. In the first step the scenarios based on their criticality to system operation are selected. The corresponding sequence diagrams are identified. The events, system states and time distribution from these sequence diagrams are then extracted. For each state, corresponding actions are identified from the statecharts.

In the second step data elements and operations corresponding to the objects active in sequence diagrams are mapped to sets of Meta-data and Meta-activity respectively.

In the third step, inputs from the actors are mapped as Meta-stimuli and outputs to actors are mapped to Meta-responses. All the operations from the objects in the tagged sequence diagrams are mapped to Meta-activities. Rules dealing with sequence diagrams extract the time distribution from the UML model.

In the fourth step, activity-response perspective of state diagrams is mapped to a child level DMM description. The connection buckets are mapped from the events and enabling conditions. Semantic queries map the events from the state diagrams to guards for enabling condition for the parent level activities. The enabling buckets are mapped from the sendEvent portion of the state transition. In the fourth step, parent level connection buckets are generated for every child level connection-bucket. The four steps are repeated until all the conflicts are removed and a final version of DMM is generated.

In the fifth step, DMM hierarchy is established by using enabling buckets on the parent as well as child levels. The mapping rules related to the five steps (described above) are given in the following paragraphs. 


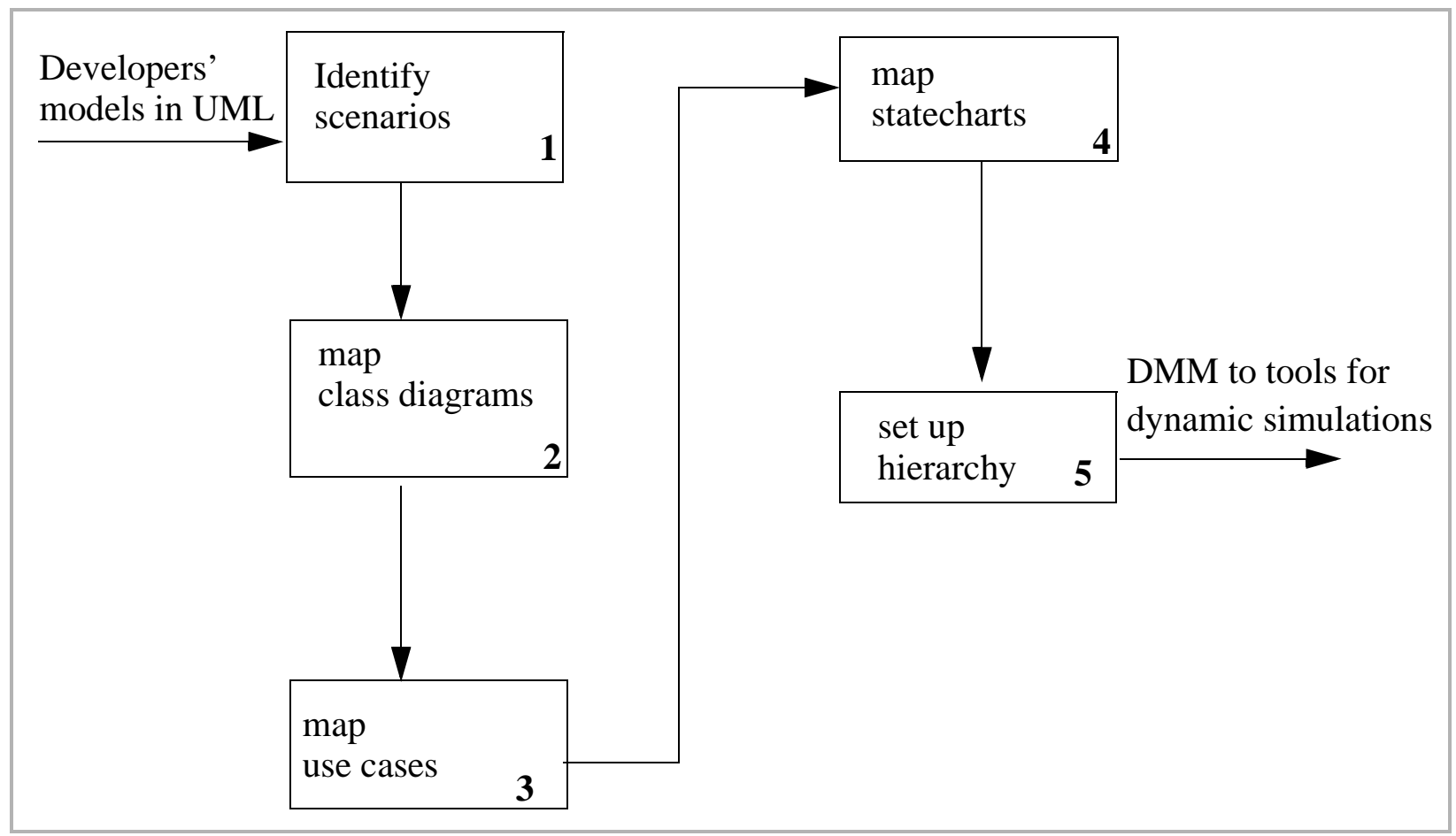

Figure 5-6 Proposed process for dynamic analysis of SART models

\section{Step:1 Identifying scenarios}

As described earlier, scenarios based on their criticality to system operation are selected. The corresponding sequence diagrams are identified.

- Rule: Identify use cases critical to the system's operation using safety requirements for the system.

- Rule: From the critical use cases, find sample scenarios with highest safety concerns.

- Rule: For the selected scenarios, identify sequence diagrams from the UML model.

\section{Step:2 Mapping the class diagrams}

Class diagrams contain elements such as attributes (of a class), operations (of a class) and messages (flowing between classes). Different types of associations further refine these elements. 
The mapping in this step is performed only for those classes whose objects are active in the sequence diagrams identified in step 1.

- $\quad$ Rule: $\forall$ class attributes $\rightarrow$ DMM data dictionary entries

- $\quad$ Rule: $\forall$ events $\rightarrow$ DMM data dictionary entries

- Rule: $\forall$ class operation $\rightarrow$ meta-activity

- Rule: $\forall$ class and operation message $\rightarrow$ meta-bucket (Based on the execution time distribution from Step 1)

From the available knowledge base, determine the average execution time for individual operations and

- Rule: $\forall$ element of execution time distribution $\rightarrow$ DMM execution time values

From sequence diagrams,

- $\quad$ Rule: $\forall$ time values $\rightarrow$ DMM $\Delta$ t (timing) distribution

The data defined for a class provide data tokens for the dynamic behavior. The operations of a class become activities for the data tokens in the dynamic behavior. The associations further refine the activities where multiple classes may define the corresponding operations. Dependencies provide the activities with specific groups of data and the related attributes. The dynamic behavior of the software systems will be effected by the class operations, attributes, structural dependency and operational dependency of classes. By analyzing the data represented by each class and the operations defined for the data, corresponding elements of the DMM are developed.

\section{Step: 3 Mapping from the use-cases}


- $\quad$ Rule: $\forall$ input from actor $\rightarrow$ meta-stimulus

- $\quad$ Rule: $\forall$ output to actor $\rightarrow$ meta-response

- $\quad$ Rule: $\forall$ DMM execution time entry $\rightarrow$ time delay for step 2 meta-activities

- Rule: $\forall$ flow of information between two nodes $\rightarrow$ meta-relation between metaactivity and meta-bucket

Use-cases provide the threads of operations in the dynamic behavior of the software systems. A thread simulates the software systems execution paths for a given set of inputs. These threads of operation are also known as scenarios. Each scenario corresponds to one instantiation of a use-case. Threads are used to link the activities in the dynamic behavior. The conditions for activating a specific thread come from interactions diagrams and the state diagrams. The dynamic behavior can be simulated by the threads, the operations spawned by these inputs and the resulting outputs.

Dynamic modeling is scenario based. Scenarios are selected for a given use-case. Interaction diagrams provide the glue binding the activities into a thread of execution paths. The scenarios affect directly how the threads will be formed. The sequence diagrams provide the timing distribution. One thing still absent from modeling the dynamic behavior is the control aspect that is provided by the information extracted from the state diagrams as discussed in the following subsection.

Time distribution may be available in two forms. First is the delta time or the time delays between different activities (object creation and destruction). The second is the time certain event occurs (messages going to or coming from the objects). Two kinds of timing information are mapped to either a Meta-activity or a Meta-relation. For the meta-activity it becomes the time delay from the moment the required number of Meta-tokens are available. After the time delay, Meta-activity generates the meta-data in its output Meta-buckets. For a Meta-relation the time 
delay has similar semantics. From the moment Meta-data is present at its input Meta-buckets, the time delay occurs before the Meta-data is transferred to its output Meta-buckets.

\section{Step 4: Mapping from the statecharts}

- $\quad$ Rule: $\forall$ superstate $\rightarrow$ meta-action (child level)

- Rule: $\forall$ state $\rightarrow$ meta-bucket

- Rule: $\forall$ transition $\rightarrow$ meta-activity

- $\quad$ Rule: $\forall$ event $\rightarrow$ meta-guard (for meta-activities above)

Based on the timing distribution from Step 1:

- Rule: $\forall \Delta \mathrm{t} \rightarrow$ time delay (for step 3 meta-activities)

- Rule: $\forall$ event occurrence $\rightarrow$ child connection-bucket (IN)

- $\quad$ Rule: $\forall$ sendEvent $\rightarrow$ child connection-bucket (OUT)

- Rule: $\forall$ (meta-activity, meta-relation) pair $\rightarrow$ meta-relation the direction of information flow

State diagrams show the control aspects of the dynamic behavior of software systems. At initialization or start-up, the system can be in an idle state, waiting for an event to occur. Once the event is present, the system will transition to a different state. While transitioning, it can activate certain operations. The system may perform certain operations when it settles in the new state. State diagrams provide the sequence of activation of different activities and probability distribution of these activities.

Statecharts model the sequence of activity and system's response to the stimuli. Their 
mapping to a DMM generates a child level. This level represents the DMM necessary to generate the Meta-data for the enabling buckets (this can also be called enabling data). The generation of enabling data depends on the system reaching a particular state (as modeled in the corresponding statechart). As mentioned earlier, a statechart is mapped to a child level. As a child, it receives system events from the parent level and provides enabling data to the parent level. If a statechart contains a superstate, a corresponding element in DMM is the Meta-action. The corresponding substate gets mapped to grand child level (or one hierarchy level below the child level). This process of generating child levels continues until we can not find any more superstate.

\section{Step 5: Setting up hierarchy in the DMM}

For corresponding parent level:

- $\quad$ Rule: $\forall$ child connection-bucket (IN) $\rightarrow$ parent connection-bucket (IN)

- $\quad$ Rule: $\forall$ child connection-bucket (OUT) $\rightarrow$ parent connection-bucket (OUT)

- $\quad$ Rule: $\forall$ child connection-bucket (IN) $\rightarrow$ parent connection-bucket (IN)

- Rule: $\forall$ flow of information between two nodes $\rightarrow$ meta-relation between metaactivity and meta-bucket

Hierarchy in a DMM is used for moving relatively primitive details to a lower or child level. This leaves the upper or parent level more readable and manageable. Flow of Meta-data between parent and child levels is represented by the parent and child connection buckets. The rules described above are used to generate the connection buckets.

The rules described in this section were applied to the UML model given in Section 5.3.1 and the resulting DMM elements were saved in a file. This file was used as an input to the process of generating CPN models as described in the following section. 


\subsection{Pacemaker example}

This section utilizes the example of a pacemaker, as described by Douglass in [52]. At the context level, this system consists of three elements, i.e., a physician (or a programmer), a pacemaker and a heart (Figure 5-7). Seven types of messages are exchanged between the three. The programmer sends out two types of messages (Commands, Magnet Applied/Removed) to a pacemaker. A pacemaker sends one message type (Pacemaker responses) to a programmer and another two types of messages (Ventricular Pace, Atrial Pace) to the heart. The heart sends two kinds of messages (Ventricular Sense, Atrial Sense) to a pacemaker. A programmer sends commands to a pacemaker through a communication link. A three letter code is used to indicate a pacemaker's operating mode. The first letter is the pacing mode (A: Atrial, V: Ventricle, D: Dual). The second letter depends on the heart chamber being used for sensing (A: Atrial, V: Ventricle, D: Dual). The third letter is for triggering (I: inhibited, T: triggered, D: Dual). Later in this section a VVI mode is used. This means the ventricle is paced, if a ventricular sense does not occur, then the pace is inhibited.

To avoid inadvertent programming by electrical noise, a magnetically activated reed switch must be closed before programming is enabled. The commands are sent to the pacemaker by pulsing an electromagnetic coil a certain number of times to indicate a 0 bit, and a different number of times to indicate a 1 bit. The commands constructed from the bits must be checked prior to acting on them, so the pacemaker should be able to send these commands back to the programmer for confirmation.

Once programmed, the pacemaker is ready to assist the heart. During this phase the pacemaker awaits for a sense event. When the heart rhythm is deemed to be irregular, the pacemaker conducts an electric current of a programmable voltage (pulse amplitude) for a programmable period of time (pulse width). After providing a pace, the pacemaker goes into a refractory state for a set period of time during which all cardiac activity is ignored. Following the 
refractory period the pacemaker resumes monitoring for the next cardiac event. The rate of pacing is programmable. The time period a pacemaker waits (after pacing), is computed based on the pacing rate and the pulse width. The refractory period is fixed.

For signals from a pacemaker to the heart, the pacemaker can pace either the atrium, the ventricular or both (dual pacing). For the signals from the heart to a pacemaker, the sense can be sent from Atrial chamber, ventricular chamber or both (dual sense).

The next section describes the UML model for this pacemaker system.

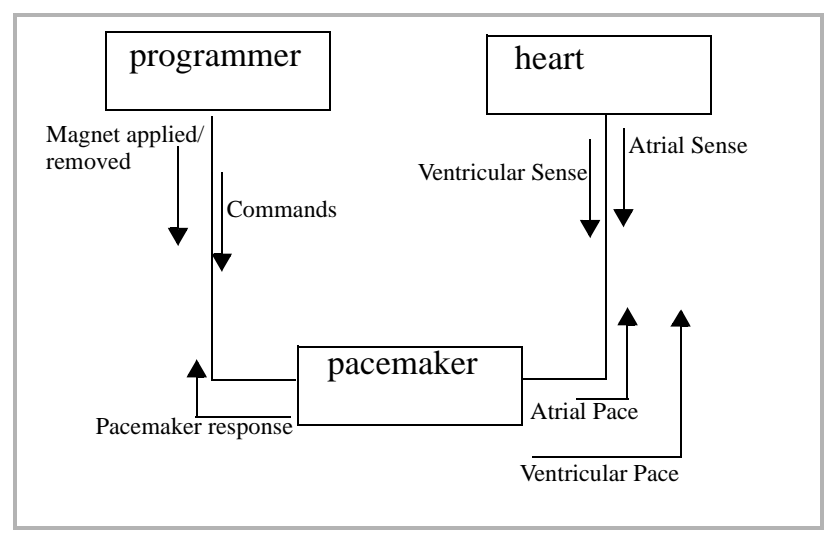

Figure 5-7 Pacemaker Context

\subsubsection{Pacemaker in UML}

From the context diagram, one can readily list three classes (of objects): programmer, pacemaker and heart. Programmer and heart are the actors in the usage of pacemaker. Therefore they are represented as two classes: programmer and heart. That leaves pacemaker class for further decomposition and subsequent analysis. Pacemaker is an aggregate class with three packages of classes (Figure 5-8): communications, pacing and battery. Communications consists of three classes: Reed switch, coil driver and communication gnome. The pacing package is an aggregate of the class chamber model and chamber's inheritances atrial model and ventricular 
model. The associations are as shown in Figure 5-8. The battery class is associated with communications gnome for providing battery status when needed.

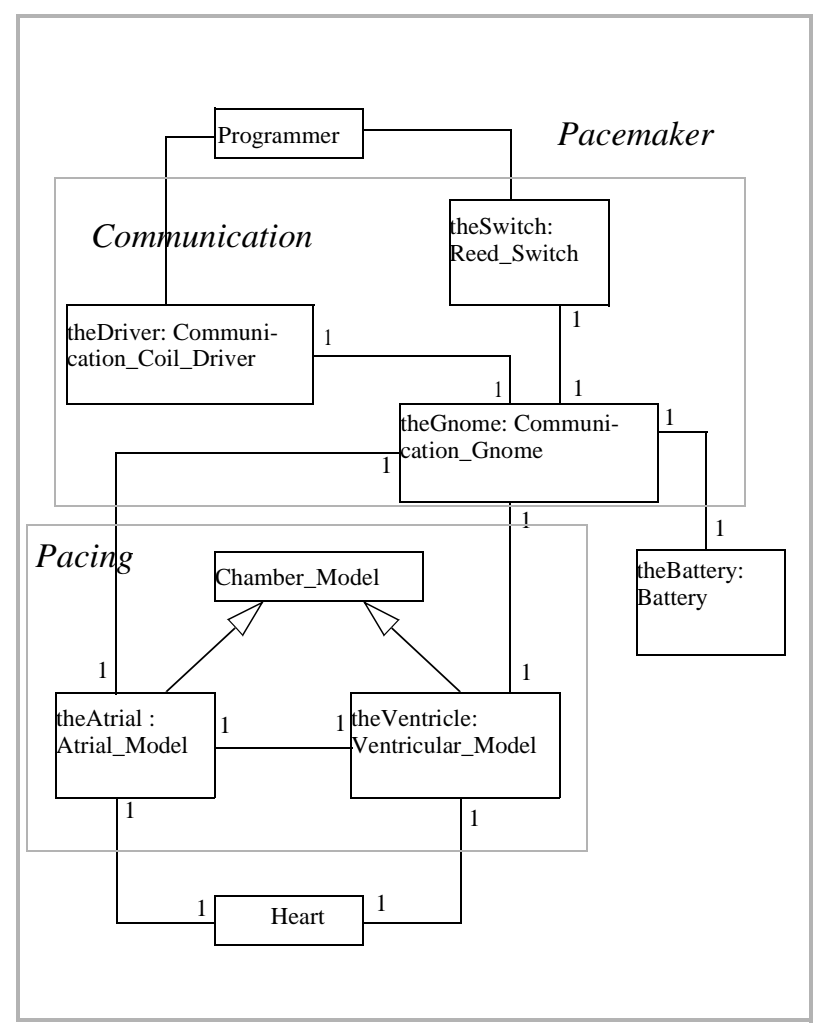

Figure 5-8 Pacemaker class diagram

The Reed switch has simple on-off state behavior (Figure 5-9) and propagates events into a communication subsystem to enable and disable communications.

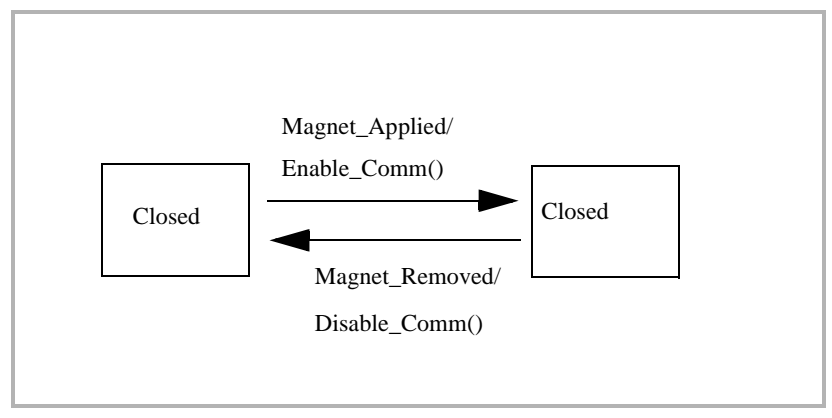

Figure 5-9 Reed Switch statechart

Coil driver's statechart is shown in Figure 5-10. When the Reed switch closes, the coil 
driver enters the idle state. From here, the coil driver could remain in idle or go to receiving incoming commands or transmitting responses. Once being in idle, if it detects pulse transition in its electromagnetic coil, it transitions to receiving_bit and waits for a timeout. If it receives another pulse transition before timing out, it increments the pulse count and restarts the timer. Eventually, when no more pulses arrive before the timeout, it transitions to the Waiting for Bit state. The actions for this transition are to decode the bit and shift the bit into the byte being constructed. If the byte is full, it is sent to the communications gnome for processing.

Transmission is enabled by the receipt of a byte to transmit from the communications gnome while the coil driver is in the idle state. The coil driver waits for a period of time in order to separate transmitted bytes. Then it begins transmitting the byte, one bit at a time. It pulses the electromagnetic coil for a specific period of time and then transitions to the conditional connector. If the guard DoneTransmitting() is true, the coil driver returns to idle. Otherwise it waits again to separate the bits in time and then sends out the next bit.

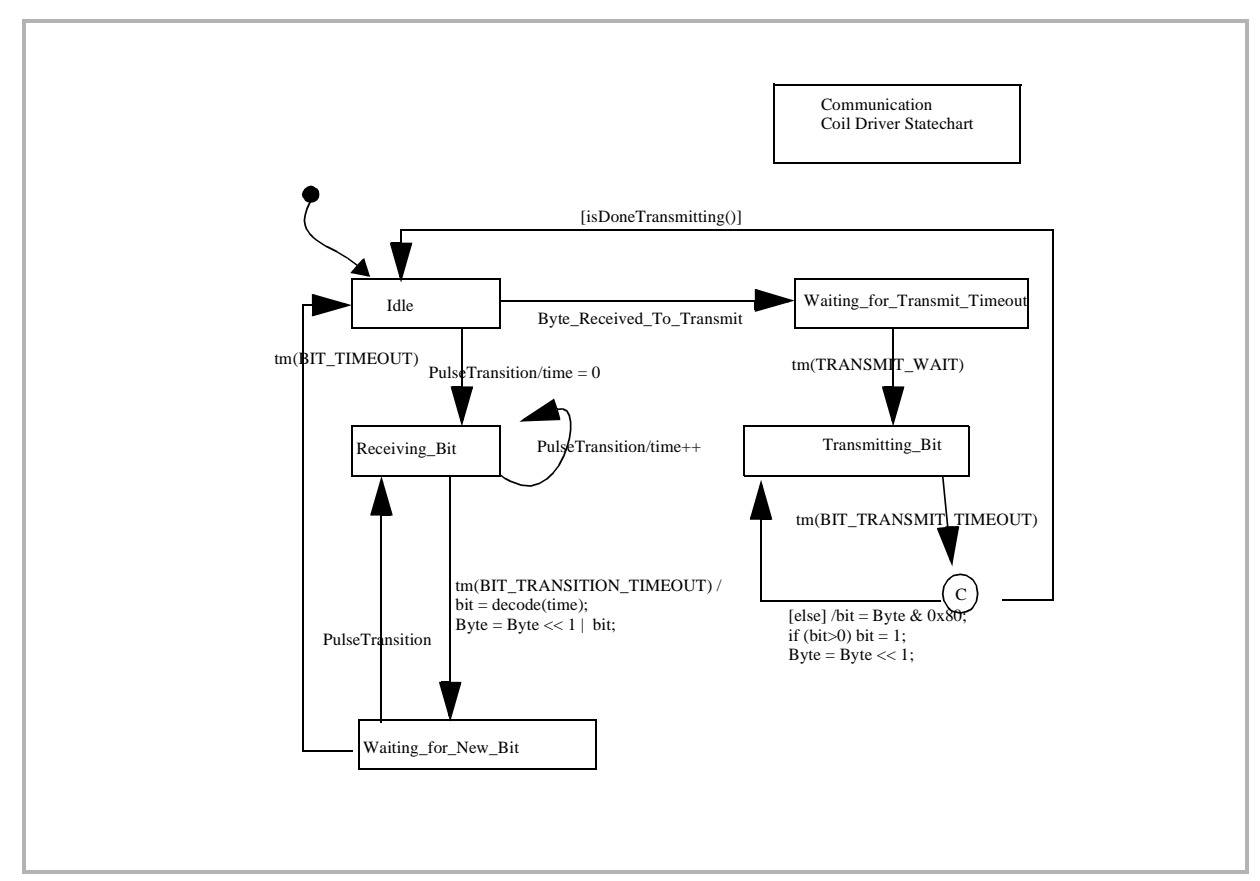

Figure 5-10 Coil driver statechart 
The statechart for the chamber model is shown in Figure 5-11. Both ventricular as well as atrial models inherit this statechart. Modal pacing behavior is modeled using the chamber model. It changes mode when commanded by the external programmer (a physician). Once commanded to be $\mathrm{ON}$, the chamber control goes to the idle state. If the communication gnome receives a command to put the pacemaker in VVI mode, it sends an inhibited event to the ventricular model object and an idle event to the atrial model. In this mode the system goes through a series of actions as shown in the sequence diagram (Figure 5-12).

The sequence diagram shows pacemaker is in an off state in the start. If commandedON is true, it transitions to idle state. If ToInhibited is true, it transitions to Refractory state. Upon entry into this state it performs an action:

if (waiting_cmd()) doIt(pending_msg);

It waits in the state until REFRACTORY_TIME (value listed in Table 5-2) expires and transitions to the state "Waiting_for_Sense". It remains in this state until the SENSE_TIMEOUT and performs an action:

Enable_Pace_Electronics (uByte Amplitude)

The pacemaker then transitions to the state "Pacing". Once the PULSE_WIDTH times out, it performs:

Disable_Pace_Electronics()

and transitions to Refractory, ready to repeat the whole cycle. 


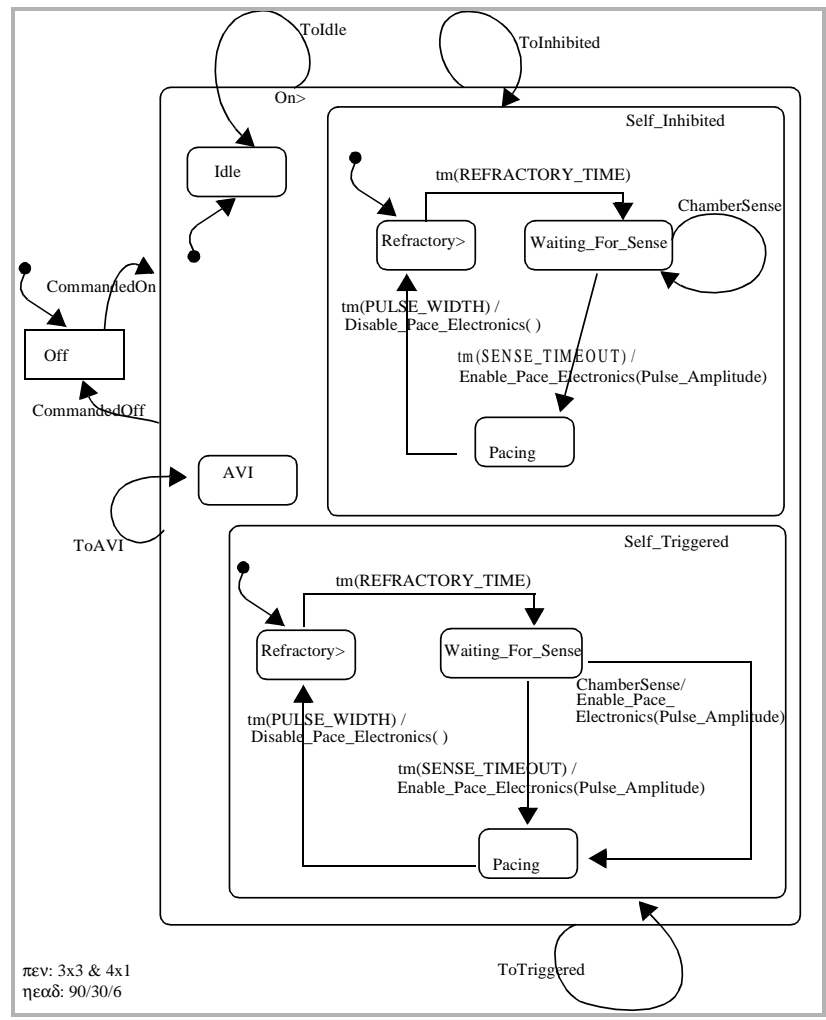

Figure 5-11 Chamber model state

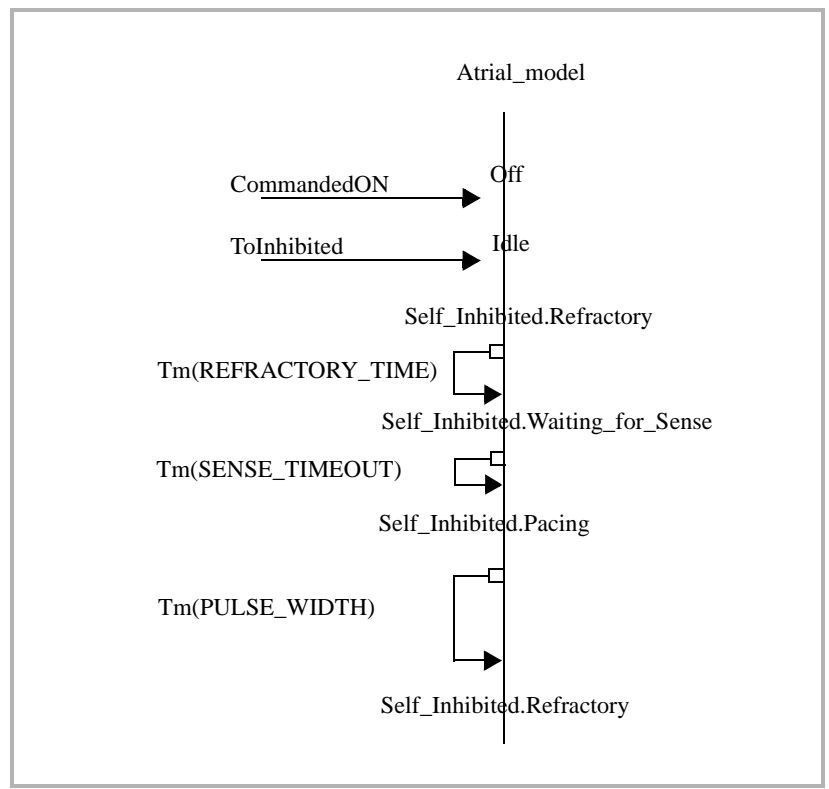

Figure 5-12 Sequence diagram (scenario for atrial model) 


\begin{tabular}{|l|l|}
\hline \multicolumn{1}{|c|}{ Variable } & \multicolumn{1}{c|}{ Definition } \\
\hline \hline msg_t & typedef unsigned char \%s[100] \\
\hline uByte & typedef unsigned char \%s \\
\hline tMsg & typedef uByte \%s[20] \\
\hline uInt & typedef unsigned int $\%$ s \\
\hline BYTE_TIME & 16 \\
\hline CMD_TIMEOUT & 18 \\
\hline BIT_TIMEOUT & 5 \\
\hline TRANSMIT_WAIT & 20 \\
\hline BIT_TRANSMIT_TIMEOUT & 5 \\
\hline BIT_TRANSITION_TIMEOUT & 5 \\
\hline SENSE_TIMEOUT & 30 \\
\hline REFRACTORY_TIME & 30 \\
\hline PULSE_WIDTH & 10 \\
\hline
\end{tabular}

Table 5-2 Variables and their definitions

\begin{tabular}{|c|c|c|}
\hline Class & Attributes & Operations \\
\hline Coil driver & $\begin{array}{l}\text { Byte: uByte } \\
\text { time : uInt } \\
\text { bit : uByte }\end{array}$ & $\begin{array}{l}\text { decode (uInt time) } \\
\text { isDoneTransmitting() } \\
\text { PulseTransition() } \\
\text { Byte_Received_To_Transmit() }\end{array}$ \\
\hline Chamber model & $\begin{array}{l}\text { Mode : int } \\
\text { Pacing_Rate : int } \\
\text { Pulse_Amplitude : int } \\
\text { Pulse_Width : int } \\
\text { pending_msg : tMsg }\end{array}$ & $\begin{array}{l}\text { Enable_Pace_Electronics(uByte amplitude) } \\
\text { Disable_Pace_Electronics() } \\
\text { doIt (tMsg cmd) } \\
\text { waiting_cmd() } \\
\text { CommandedOn() } \\
\text { CommandedOff() } \\
\text { ToSelfInhibit() } \\
\text { ToIdle() } \\
\text { ToTriggered() } \\
\text { ToInhibited() } \\
\text { ToAVI() } \\
\text { DonePacing() } \\
\text { ChamberSense() } \\
\text { HerezaCmd(uByte* pMsg) }\end{array}$ \\
\hline $\begin{array}{l}\text { Atrium/Ventricu- } \\
\text { lar model }\end{array}$ & same as chamber model & $\begin{array}{l}\text { chamber + } \\
\text { V_Sense() }\end{array}$ \\
\hline $\begin{array}{l}\text { Communications } \\
\text { gnome }\end{array}$ & $\begin{array}{l}\text { msg_len : uInt } \\
\text { msg : tMsg }\end{array}$ & $\begin{array}{l}\text { isDoneTransmitting() } \\
\text { isValid(tMsg Cmd) } \\
\text { validate(tMsg Cmd) } \\
\text { Handle_Cmd(tMsg msg) } \\
\text { Disable_Comm() } \\
\text { Bit_Received() } \\
\text { Cmd_Ready_to_Transmit() } \\
\text { Enable_Comm() } \\
\text { Byte_Received() }\end{array}$ \\
\hline
\end{tabular}

Table 5-3 Pacemaker model: Classes, Attributes \& Operations 


\begin{tabular}{|l|l|l|}
\hline \multicolumn{1}{|c|}{ Class } & \multicolumn{1}{|c|}{ Attributes } & \multicolumn{1}{c|}{ Operations } \\
\hline \hline $\begin{array}{l}\text { Communications } \\
\text { gnome: } \\
\text { Transmit_Queue: } \\
\text { Queue }\end{array}$ & $\begin{array}{l}\text { size } \\
\text { head } \\
\text { tail }\end{array}$ & $\begin{array}{l}\text { enqueue (unsigned int length, unsigned char cmd[100]) } \\
\text { dequeue (unsigned int* length, unsigned char cmd[100]) }\end{array}$ \\
\hline $\begin{array}{l}\text { Communications } \\
\text { gnome: } \\
\text { Receive_Queue } \\
\text { Queue }\end{array}$ & $\begin{array}{l}\text { size } \\
\text { head } \\
\text { tail }\end{array}$ & $\begin{array}{l}\text { enqueue (unsigned int length, unsigned char cmd[100]) } \\
\text { dequeue (unsigned int* length, unsigned char cmd[100]) }\end{array}$ \\
\hline Reed_Switch & & $\begin{array}{l}\text { Magnet_Applied() } \\
\text { Magnet_Removed() }\end{array}$ \\
\hline
\end{tabular}

\section{Table 5-3 Pacemaker model: Classes, Attributes \& Operations}

\subsubsection{DMM model}

The atrial scenario is used to develop a DMM. It consists of a parent level DMM and one child level DMM. In the parent level DMM, stimuli/responses external to the scenario are supplied. The stimuli are deposited in Meta-buckets and become input to the meta-action representing the scenario. The responses are deposited in Meta-buckets (parent connection buckets) attached to the output of the same Meta-action. This is summarized in Table 5-4 and Table 5-5. Symbols used for different elements of DMM are listed in Table 5-1.

At the child level the input Meta-stimuli are CommandedON, ToInhibited and ChamberSense. The output Meta-responses are enable_pace_electronics and disable_pace_electronics. Enabling Meta-buckets are used to store the stimuli and responses. All the states in this scenario ( 5 in number), input events ( 3 in number) and output events ( 2 in number) are mapped to Meta-buckets (Table 5-6). There are no Meta-actions at the child level. Meta-activities are listed in Table 5-7. Meta-function F1 performs the following task:

if (waiting_cmd()) then $\operatorname{doIt}()$;

For each pair of tables (meta-buckets and meta-activities), meta-relations are used to connect the bucket-activity pair. Tables 5-1 through 5-4 are used to develop a CPN diagram. 


\begin{tabular}{|c|c|c|c|}
\hline S. No. & Meta-buckets & Description & Initial Value \\
\hline 1 & $c b_{p-m}(\mathrm{IN})$ & input connection-bucket: CommandedON & 0 \\
\hline 2 & $c b_{p-m}(\mathbf{I N})$ & input connection-bucket: ToInhibited & 0 \\
\hline 3 & $c b_{p-m}(\mathbf{I N})$ & input connection bucket: chamberSense & 0 \\
\hline 4 & $c b_{p-m}($ OUT $)$ & output connection bucket: enable_pace_electronics & 0 \\
\hline 5 & $c b_{p-m}($ OUT $)$ & output connection bucket: disable_pace_electronics & 0 \\
\hline
\end{tabular}

Table 5-4 Meta-buckets for the parent level DMM

\begin{tabular}{|c|l|l|l|l|l|l|}
\hline $\begin{array}{c}\text { S. } \\
\text { No. }\end{array}$ & $\begin{array}{c}\text { Meta- } \\
\text { activity } \\
\text { / Action }\end{array}$ & $\begin{array}{c}\text { input } \\
\mathbf{B}_{m}\end{array}$ & $\begin{array}{c}\text { outp } \\
\text { ut } \\
\boldsymbol{B}_{\boldsymbol{m}}\end{array}$ & $\begin{array}{c}\text { delta } \\
\text { Time } \\
\text { (delay) }\end{array}$ & $\begin{array}{c}\text { Meta } \\
- \\
\text { funct } \\
\text { ion }\end{array}$ & $\begin{array}{c}\text { Meta- } \\
\text { guard }\end{array}$ \\
\hline \hline $\mathbf{1}$ & $\boldsymbol{A}_{\boldsymbol{m}}$ & $1,2,3$ & 4,5 & 0 & $\mathrm{n} / \mathrm{a}$ & $\mathrm{n} / \mathrm{a}$ \\
\hline
\end{tabular}

Table 5-5 Meta-activities/Actions for parent level DMM

\begin{tabular}{|l|l|l|l|l|}
\hline \multicolumn{1}{|c|}{ S. No. } & \multicolumn{1}{|c|}{ Meta-buckets } & \multicolumn{1}{c|}{ Description } & Time delay & Initial Value \\
\hline \hline $\mathbf{1 .}$ & $\boldsymbol{B}_{\boldsymbol{m}}$ & state: Off & 0 & 0 \\
\hline $\mathbf{2}$ & $\boldsymbol{c} \boldsymbol{b}_{\boldsymbol{c}-\boldsymbol{m}}(\mathbf{I N})$ & input connection-bucket: CommandedON & 0 & 0 \\
\hline $\mathbf{3}$ & $\boldsymbol{B}_{\boldsymbol{m}}$ & state: idle & 0 & 0 \\
\hline $\mathbf{4}$ & $\boldsymbol{c} \boldsymbol{b}_{\boldsymbol{c}-\boldsymbol{m}}(\mathbf{I N})$ & input connection-bucket: ToInhibited & 0 & 0 \\
\hline $\mathbf{5}$ & $\boldsymbol{B}_{\boldsymbol{m}}$ & state: Refractory & 0 & 0 \\
\hline $\mathbf{6}$ & $\boldsymbol{B}_{\boldsymbol{m}}$ & state: Waiting_For_Sense & 0 & 0 \\
\hline $\mathbf{7}$ & $\boldsymbol{c} \boldsymbol{b}_{\boldsymbol{m}}(\mathbf{I N})$ & 0 & 0 \\
\hline $\mathbf{8}$ & $\boldsymbol{c} \boldsymbol{b}_{\boldsymbol{m}}(\mathbf{O U T})$ & input connection bucket: chamberSense & 0 \\
\hline $\mathbf{9}$ & $\boldsymbol{B}_{\boldsymbol{m}}$ & output connection bucket: enable_pace_electronics & & 0 \\
\hline $\mathbf{1 0}$ & $\boldsymbol{c} \boldsymbol{b}_{\boldsymbol{m}}(\mathbf{O U T})$ & state: Pacing & & 0 \\
\hline
\end{tabular}

Table 5-6 Meta-buckets for the child level DMM

\begin{tabular}{|l|l|l|l|l|l|l|}
\hline \multicolumn{1}{|c|}{ S. No. } & \multicolumn{1}{c|}{$\begin{array}{c}\text { Meta-activity / } \\
\text { Action }\end{array}$} & \multicolumn{1}{|c|}{ input $\mathbf{B}_{\boldsymbol{m}}$} & \multicolumn{1}{c|}{ output $\boldsymbol{B}_{\boldsymbol{m}}$} & \multicolumn{1}{c|}{ delta Time (delay) } & Meta-function & Meta-guard \\
\hline \hline $\mathbf{1}$ & $\boldsymbol{a}_{\boldsymbol{m}}$ & 1,2 & 3 & 0 & $\mathrm{n} / \mathrm{a}$ & CommandedON $=$ True \\
\hline $\mathbf{2}$ & $\boldsymbol{a}_{\boldsymbol{m}}$ & 3,4 & 5 & 0 & $\mathrm{~F}_{1}$ & ToInhibited $=$ True \\
\hline $\mathbf{3}$ & $\boldsymbol{a}_{\boldsymbol{m}}$ & 5 & 6 & REFRECTORY_TIME & & \\
\hline $\mathbf{4}$ & $a_{\boldsymbol{m}}$ & 6,7 & 6 & 0 & & ChamberSense $=$ True \\
\hline
\end{tabular}

Table 5-7 Meta-activities/Actions for child level DMM 


\begin{tabular}{|l|l|l|l|l|l|l|}
\hline S. No. & \multicolumn{1}{|c|}{$\begin{array}{c}\text { Meta-activity / } \\
\text { Action }\end{array}$} & \multicolumn{1}{c|}{ input $\mathbf{B}_{\boldsymbol{m}}$} & \multicolumn{1}{c|}{ output $\boldsymbol{B}_{\boldsymbol{m}}$} & \multicolumn{1}{c|}{ delta Time (delay) } & Meta-function & Meta-guard \\
\hline \hline $\mathbf{5}$ & $\boldsymbol{a}_{\boldsymbol{m}}$ & 6 & 8,9 & SENSE_TIMEOUT & & \\
\hline $\mathbf{6}$ & $\boldsymbol{a}_{\boldsymbol{m}}$ & 9 & 10,5 & PULSE_WIDTH & $\mathrm{F}_{1}$ & \\
\hline
\end{tabular}

Table 5-7 Meta-activities/Actions for child level DMM 5.4 Generating CPN models from DMM

Design/CPN will be used for implementing DMM in a simulation capable DPN environment. Although Design/CPN lacks some of the requirements of DMM, CPN features (like code section and substitution transitions) can be used to overcome some of its shortcomings.

All the work performed in building a DMM pays off in the form of a relatively simple process of generating CPN models from DMM. The DMM data dictionary at the global level becomes a global declaration page of the CPN model. The local level data dictionary becomes a local declaration page. The Meta-data is used to create CPN data tokens. Meta-activities become transitions and Meta-buckets as places.

DMM Meta-relations are the arcs in CPN. The color of places is based on the DMM data dictionary declarations. Meta-actions become substitution transitions and the connection-buckets are the ports and sockets of a CPN model. The child level DMM is a sub-page of the CPN and the parent level DMM is a super-page. A Design/CPN tool allows for using time values for transitions so the time delay and execution time distributions of DMM will be used in defining time values for the CPN transitions and arcs. Using the process described in this section, a CPN model was generated using Design/CPN. Some of the sections of this CPN model are given in Figure 5-13 and Figure 5-14.

Figure 5-13 shows a CPN model for the Atrium scenario. There are four input places, i.e., Cmd, CommandedON, ToInhibited, ChamberSense. There are four output places, i.e., enable_pace_electronics, disable_pace_electronics, enable_doIt. The lone transition in the figure is the substitution transition (HS or hierarchical substitution). In the CPN hierarchy, Figure 5-13 
represents the superpage with a corresponding subpage as described in the following paragraph.

Figure 5-14 shows the subpage for the substitution transitions discussed in the paragraph above. The places marked with the letter "P" in a rectangle are known as ports. Port-socket pairs link superpages and subpages so as to implement substitution transitions. Superpages and subpages are connected by pairs of places on the two pages using special purpose fusion sets. A place that belongs to such a fusion set is called a port if it is on a subpage, and a socket if it is on a superpage.

For simplicity the ports-sockets pairs are given the same name. The place named "Off" has one token by default. When CommandedOn gets a token, the transition $\mathrm{T}_{1}$ fires and one token each gets deposited in "Off" and "Idle". The token in "Off" will be used when "CommandedOn" gets the next token. Once "Idle" has a token and "ToInhibited" receives a token as well, the transition $\mathrm{T}_{2}$ fires and one token gets deposited in "Refractory". An action on entry to "Refractory" is performed as

if (waiting_cmd()) doIt(pending_msg).

This action is represented by a code segment " $\mathrm{C}$ " attached to the transition $\mathrm{T}_{2}$. After waiting for the REFRECTORY_TIME timeout, $\mathrm{T}_{3}$ fires and a token gets deposited in the place "waiting_For_sense". If the stimulus "ChamberSense" is present, $\mathrm{T}_{4}$ will remove a token from "waiting_For_sense" and then deposit one token back in the same place. This action shows that the system remains in the same state if the stimulus "ChamberSense" is provided to the system. Once a token is present in "waiting_For_sense" long enough for the time out SENSE_TIMEOUT to expire, transition $\mathrm{T}_{5}$ fires and a token each is deposited in "enable_pace_electronics" (an output port) and "Pacing". The port "enable_pace_electronics" in subpage (Figure 5-14) supplies the deposited token to its socket "enable_pace_electronics" present in the superpage shown in Figure 5-13, where it can be consumed by an external sink (not modeled). Once a token in "Pacing" is 
present until the time out PULSE_WIDTH, the transition $\mathrm{T}_{6}$ fires and deposits a token in "Refractory". When $\mathrm{T}_{4}$ fires, a code segment gets executed to perform the following action:

if (waiting_cmd()) doIt(pending_msg).

Once in "Refractory", the system continues to function in a loop.

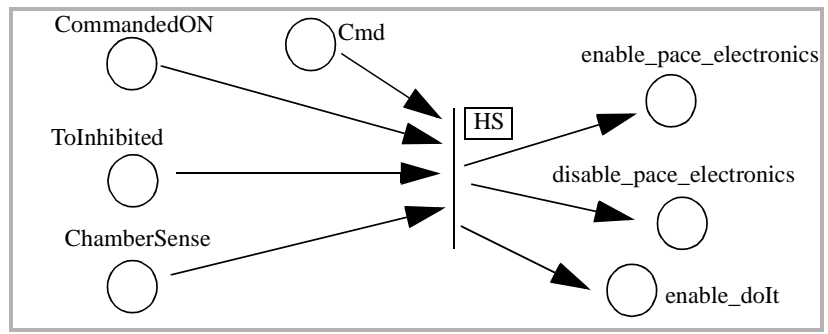

Figure 5-13 CPN model for Atrium Scenario

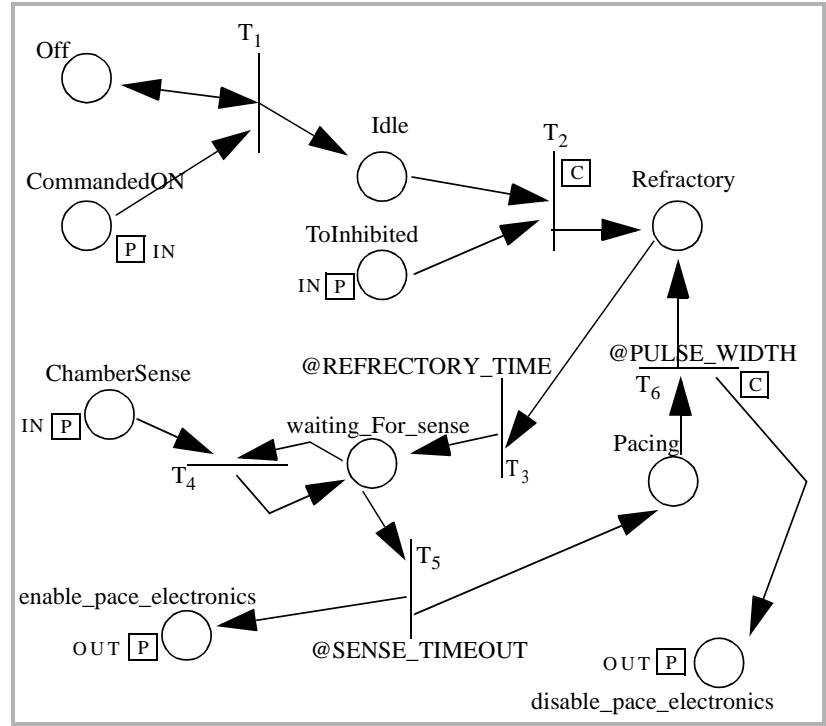

Figure 5-14 Substitution CPN diagram for Atrium Scenario.

A framework for dynamic analysis of UML models was presented in this chapter. The following chapter describes application of SART related framework to dynamic analysis of a large scale software system. 


\section{Chapter 6 Application of Methodology to a Large Software System}

This chapter presents a case study as a proof of concept for the method presented in Chapter 4. In that chapter, mapping rules were developed for transferring semantics of SART notation to CPN. Those mapping rules were used to develop a semantics translation utility. In the following sections this utility is used to map a large software system to CPN.

This large scale software system is called EOS (Earth Observation System), also known as "Mission to Planet Earth", will be deployed by NASA (National Aeronautics and Space Administration). The first reason why this system was selected for the case study is, because it is an industrial scale system. The second reason is, that it contains a large real time component called FOS (Flight Operation Segment). The third reason is all the necessary documentation for EOS was available for this project under a grant from NASA to West Virginia University. The following sections describe the EOS system and show the analysis performed on it using the techniques of the earlier chapters.

\subsection{Description of the Commanding subsystem of EOS}

EOS is a large scale PDS (Parallel and distributed system). NASA plans to launch a total of six earth-looking scientific observatories accompanied by other observatories from the European Space Agency. The instrumentation will focus primarily on measurements designed to enhance our understanding of earth's climate, hydrologic systems and ecosystem dynamics. The context diagram of FOS is shown in Figure 6-1. Solid lines represent the flow of data and the dotted lines represent the flow of control signals. FOS is responsible for receiving data from and sending commands to nine major subsystems in EOS. The data from these subsystems is necessary for generating the uplink commands (commands to be sent to the satellites), simulation and archiving. FOS interfaces with EDOS (EOS Data and Operations Systems) for the command 
uplink and receives the realtime command from and displays the messages to the operators. An operator can be a project scientist or designee, operations manager, principal investigator, team leader and user. The interaction of FOS with external entities is shown in Figure 6-1. Modeling FOS is a labor-intensive process. The SART model of FOS considered for this work consists of 85 diagrams (48 DFDs and 37 STDs) to define the C-specs. The description of the processes in DFD0 goes to a maximum of five levels deep in the hierarchy. The module selected for dynamic modeling is "Commanding" process.

Based on the requirement specifications and scheduling scenario, it was observed the commanding module plays an important role. The location of Commanding in the hierarchy of FOS is shown in Figure 6-2. Commanding is a child DFD of Command_Generation, a subfunction of EOC (EOS Operations Center).

A model of the Commanding Subsystem was built based on the requirement specifications of NASA. The Commanding module performs four major tasks as shown in Figure 6-2 and listed below:

- Generate and verify realtime commands. This is accomplished by the functions Build_Spacecraft_Realtime_Command and Verify_Command [53].

- Merge and uplink the pre-planned and realtime commands to EDOS. The functions Merge_Command and Transmission_Command are responsible for this job [53].

- Receive and evaluate the command status. This is done by the functions: Evaluate_ Spacecraft_Command_Status and Receive_Command_Status_Data [53].

- Automatic retransmission is provided when an unsuccessful transmission occurs. This is managed with the help of the function Count_Transmission_ number [54]. 


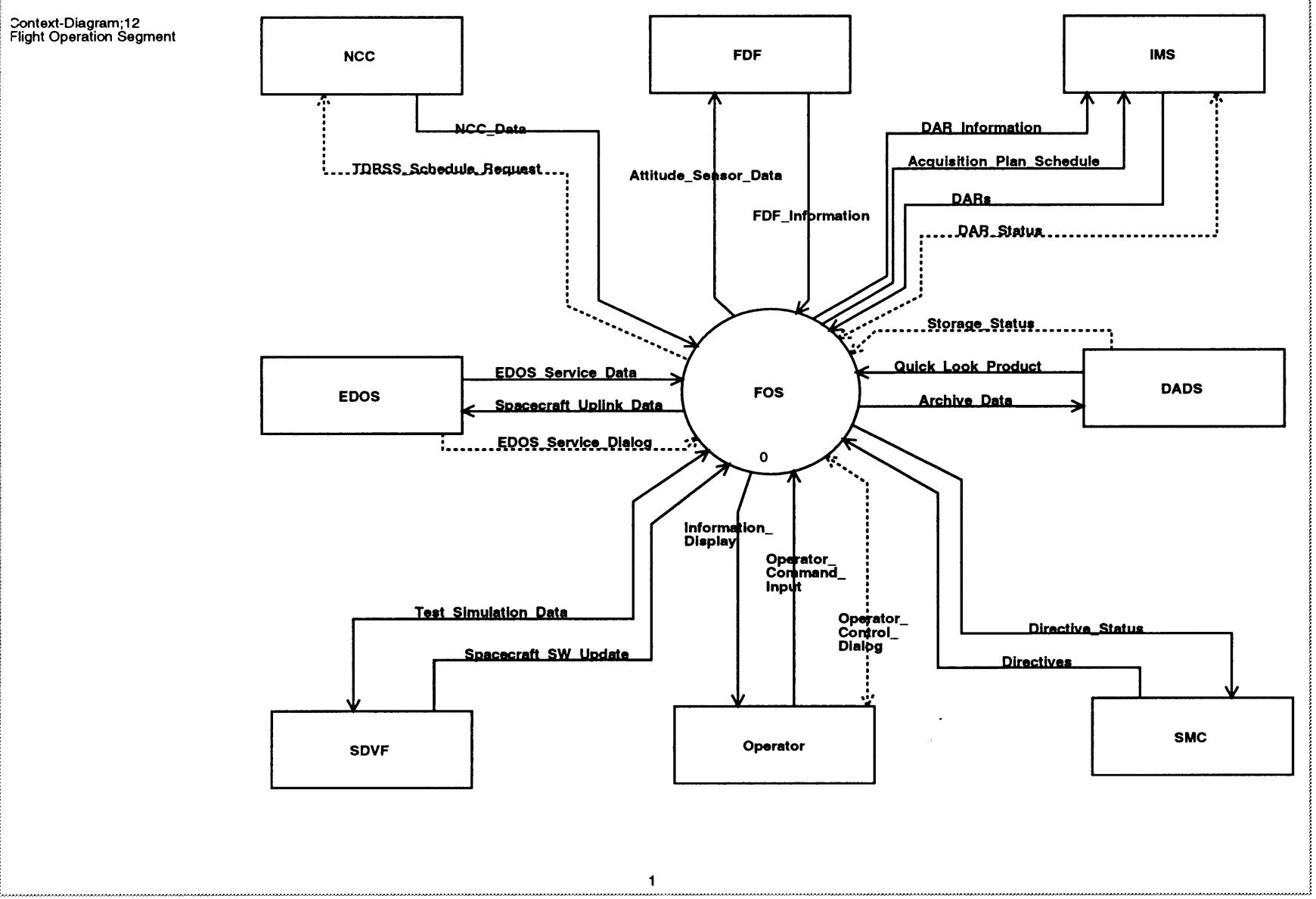

Figure 6-1 The Context Diagram for FOS

To fulfill the above mentioned tasks, Commanding is decomposed into several subfunctions:

- Build_Spacecraft_Realtime_Command,

- Verify_Command,

- Merge_Command,

- Transmit_Command, 
- Evaluate_Spacecraft_Command_Status,

- Receive_Command_Status_Data,

- Count_Transmission_number.

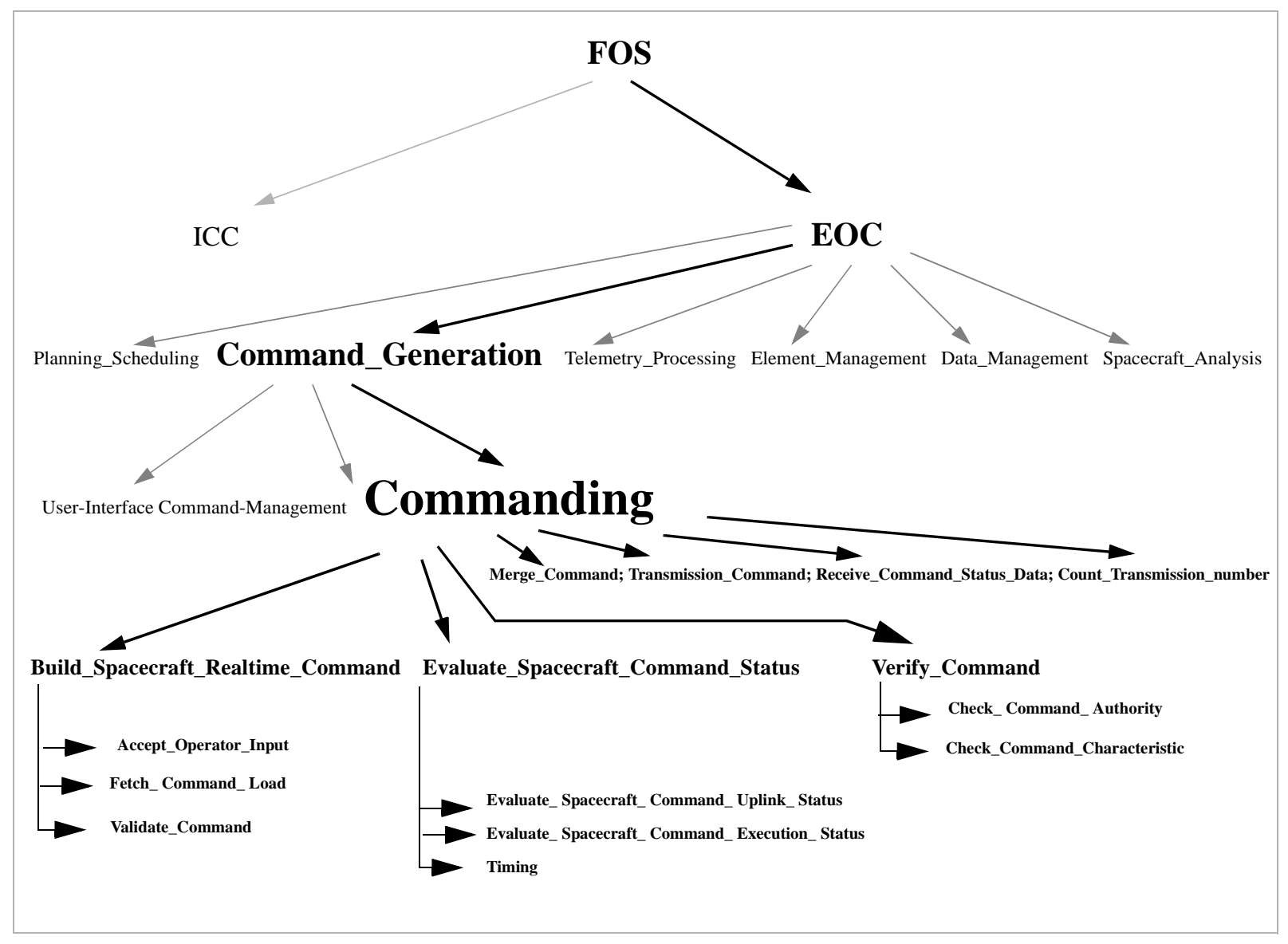

Figure 6-2 Location of various modules in the EOS DIS system.

The above subfunctions can be activated concurrently to build and uplink a stream command. The Commanding DFD is shown in Figure 6-3 and the Controller for Commanding is shown in Figure 6-6. Out of the seven subfunctions of Commanding, four are relatively simple:

- Merge_Command merges a Valid_Realtime_Command and a Valid_Preplanned_Command. 
- Transmit_Command receives Uplink_Data_Stream from the Merge_Command subfunction and sends it to the space crafts as a Spacecraft_Uplink_Data.

- Count_Transmission_Number controls the retransmission efforts needed when the data received from the space craft indicate the command has been rejected.

- Receive_Command_Status_Data is used to monitor the status of data received by the space craft.

The other three subfunctions are relatively more complex: and are described in the following subsections.

\subsubsection{Build_Spacecraft_Realtime_Command}

Build_Spacecraft_Realtime_Command generates the spacecraft realtime command based on the operator command input. The pre-planned command script "Accept_Operator_Input" accepts the operator command input. Fetch_CCommand_Load" fetches the realtime command from the pre-planned command script according to the index. The realtime command will be validated by a Validate_Command subfunction.

\subsubsection{Verify_Command}

Verify_Command checks the authorization level of a command and determines whether a specific command is critical based on its definition [53]. The authorization level checking is performed by a Check_CCommand_Authority subfunction and the critical command confirmation is realized by a Check_Command_Characteristic subfunction.

\subsubsection{Evaluate_Spacecraft_Command_Status}

The Evaluate_Spacecraft_Command_Status function verifies the successful receipt and execution of all commands by the spacecraft [53]. Both evaluations are based on telemetry data. 
The function Evaluate__Spacecraft_ Command__Uplink_ Status evaluates the spacecraft command uplink status to verify the successful receipt. The process Evaluate_ Spacecraft_ Command_Execution_Status evaluates the execution status of the Spacecraft_commands by the spacecraft itself. 


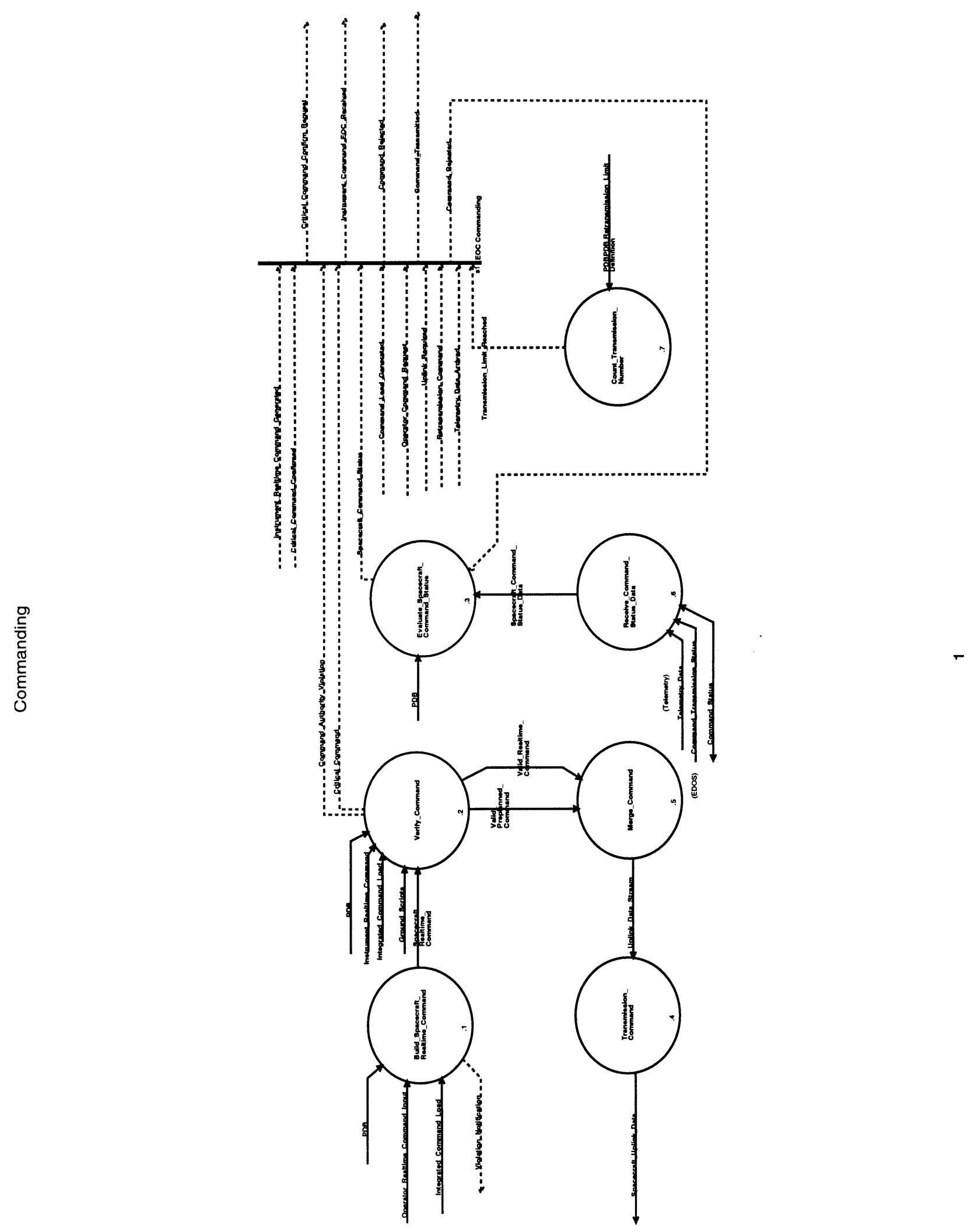

Figure 6-3 DFD 1.2.3 Commanding process 

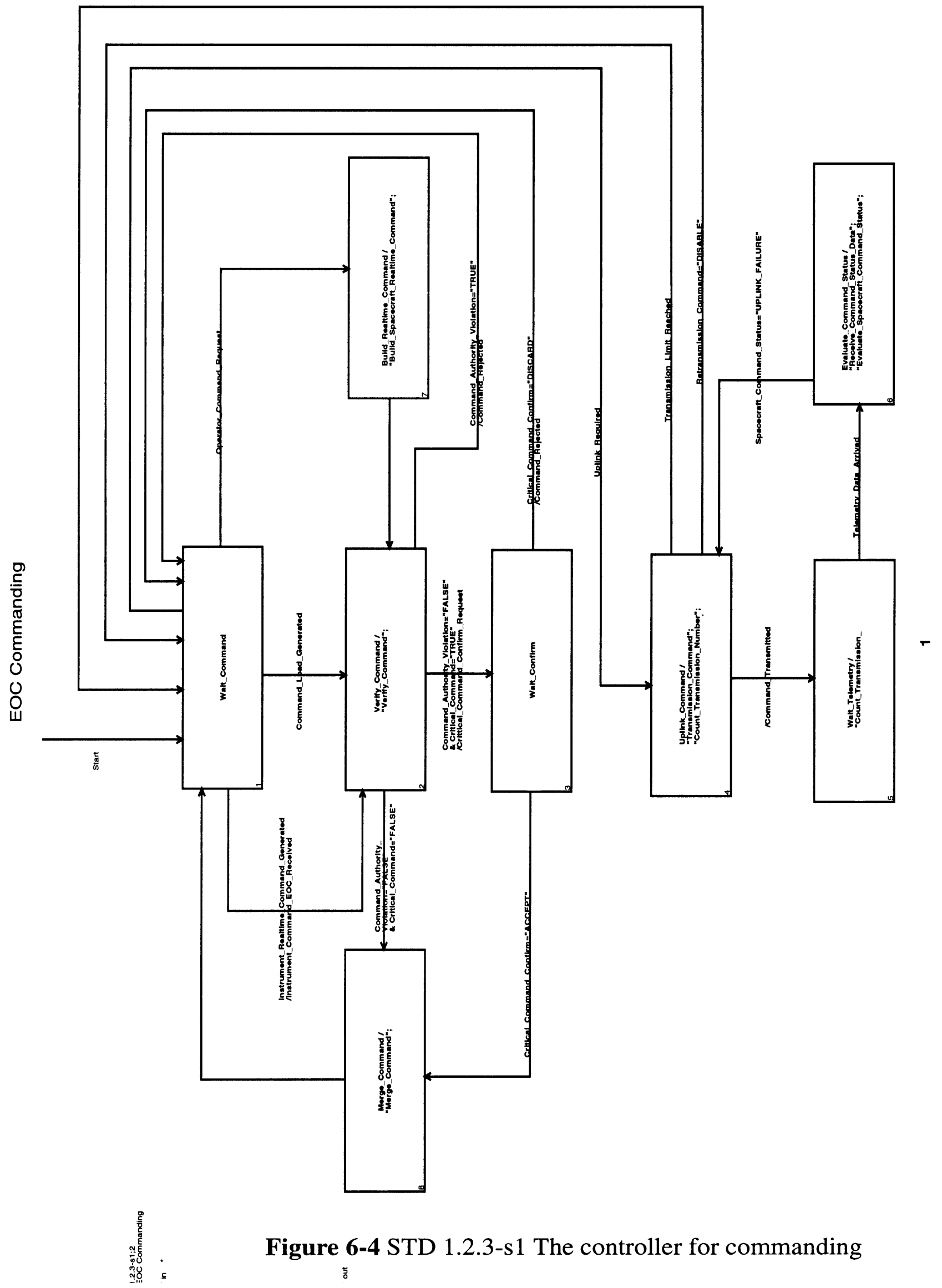

Figure 6-4 STD 1.2.3-s1 The controller for commanding 


\subsection{Dynamic Modeling of the Commanding subsystem}

The model of the Commanding subsystem, as described in the previous section, was built using the CASE tool Teamwork. The Teamwork model of Commanding was translated to the Design/CPN environment for dynamic analysis. The translation was preformed by mapping the semantics from Teamwork to Design/CPN as described in [41]. The mapping of CPN pages from the DFDs and STDs of the Commanding system is listed in Table 6-1. The Hierarchy page of the CPN model is shown in Figure 6-5.

\begin{tabular}{|c|c|c|}
\hline $\begin{array}{r}\text { CPN } \\
\text { Page } \\
\text { No. }\end{array}$ & $\begin{array}{c}\text { CPN Page } \\
\text { Name }\end{array}$ & Mapped from DFDs and STDs \\
\hline \hline 1 & Glob Dec & Global Declaration page for the CPN model. \\
\hline 2 & Commanding & This is mapped from DFD 1.2.3 “Commanding” function. \\
\hline 3 & BuildSRC & DFD 1.2.3.1.Build_Spacecraft_Realtime_Command” function. \\
\hline 4 & VeriComm & DFD-1.2.3.2.Verify_Command” function. \\
\hline 5 & EvalSCS & DFD-1.2.3.3 "Evaluate_Spacecraft_Command_Status" function. \\
\hline 6 & BSRC-Con & STD-1.2.3.1-s1Control_Build_Spacecraft_Realtime_Command \\
\hline 7 & VCom-Con & STD-1.2.3.2-s1“Control_Verify_Command”C-Spec. \\
\hline 8 & ESCS_Con & STD-1.2.3.3-s1“Control_Evaluate_Spacecraft_Command_Status” \\
\hline 9 & EOC_Comm & STD-1.2.3-s1 “Control_EOC_Commanding”C-Spec. \\
\hline
\end{tabular}

Table 6-1 Mapping of CPN pages to various DFDs and STDs

The CPN model mapped from the Teamwork model needs to be completed by adding the missing semantics needed for dynamic analysis. The declaration page needs to be updated to add any missing type declarations. CPN Meta Language code is written to map the outputs from the inputs. The information needed to implement a particular scenario is also added to the model to carry out the execution of the scenario. Thus the model is customized for each simulation and the behavior of the model is analyzed under different scenarios. 


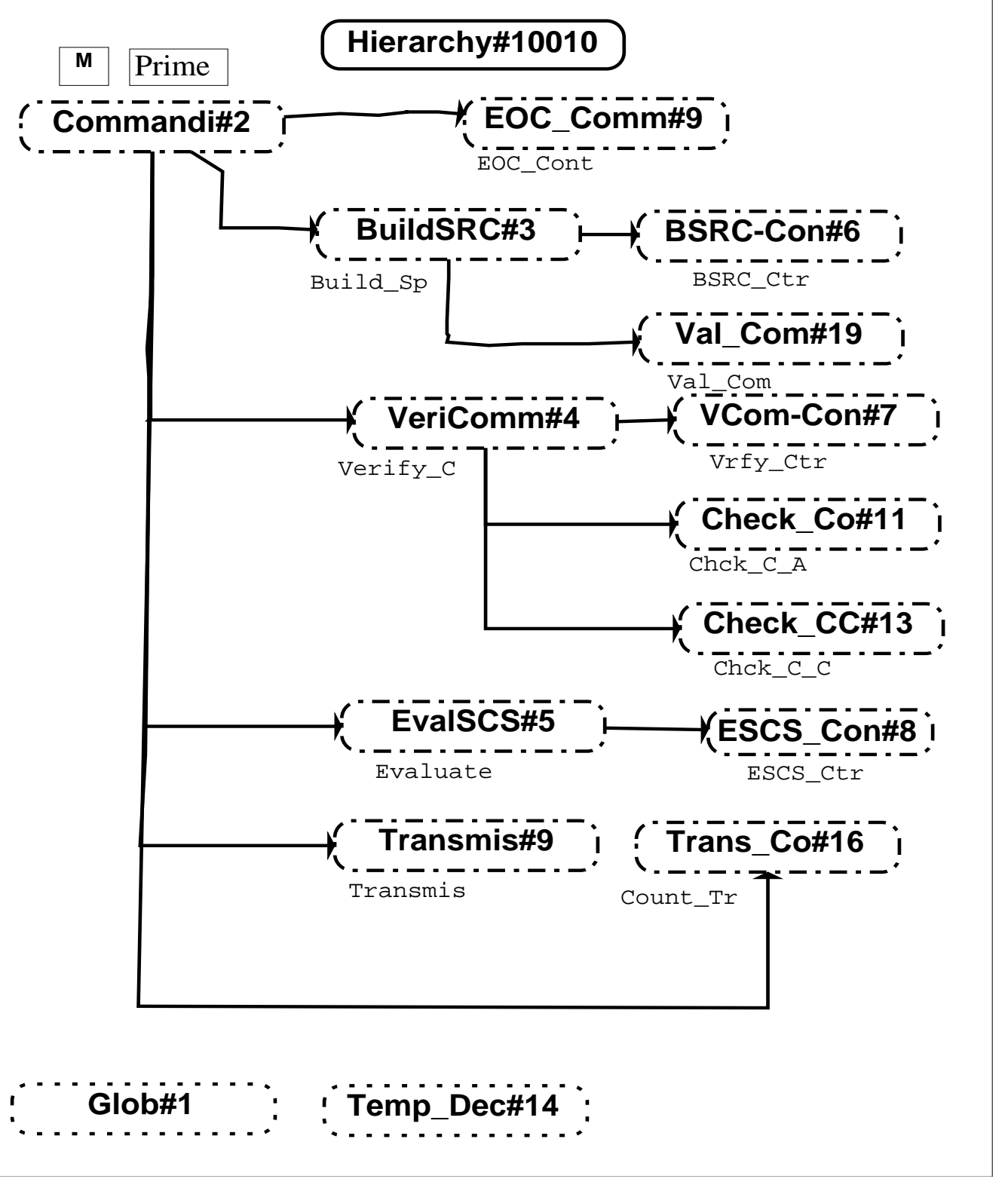

Figure 6-5 The hierarchy page of CPN model.

The CPN page corresponding to the Commanding DFD is shown in Figure 6-6. This page has been mapped from the DFD shown in Figure 6-3. The transitions on these pages (Build_Spacecraft_Realtime_Command, Verify_Command, Merge_Command, Transmission_Command, Count_Transmission_Number, Receive_Command_Status_Data, 
Evaluate_Spacecraft_Command_Status and the EOC_Controller) are mapped from the corresponding functions in the Teamwork model. The transition Spacecraft simulates the Spacecraft responses for the transmitted commands.

The Build_Spacecraft_Realtime_Command (BSRC) transition takes as input Operator_Command_Input and processes it to produce Spacecraft_Realtime_Command. It contains three submodules: Accept_Operator_Input, Fetch_Command_Load and Validate_Command. The Accept_Operator_Input function accepts the input command from the operator. If there was a request for Command_Load from the project database then the Fetch_Command_Load function is invoked to fetch a sequence of commands indicated by the Command_Load_Index variable. When a command is generated it is validated by the Validate_Command function and if a realtime violation is detected then a Violation_Notification signal is sent to the operator.

Verify_Command takes as input either a Spacecraft_Realtime_Command generated by the BSRC module or an Instrument_Realtime_Command or an Integrated_Command_Load that are sent by the ICC (Instrument Control Center). It has two submodules: Check_Command_Authority and Check_Command_Characteristic. Check_Command_Authority checks if the issuer of the Command has the necessary permissions to execute the command and signals a Command_Authority_Violation if the authority is invalid. The Check_Command_Characteristic function checks if the Command being uplinked is a critical command. For a critical command a second positive response from the operator is requested before the Command is uplinked.

The Merge_Command function merges the Valid_Preplanned_Commands and Valid_Realtime_Commands resulting from the Verify_Command function into one Uplink_Data_Stream. In the code segment associated with the Merge_Command transition the Uplink_Data_Stream token is generated, where the uplink time is noted and the down link time is initialized to zero. Downlink time represents the time it takes for spacecraft data to reach the 
receiving station on the ground.

The Transmission_Command function takes the Uplink_Data_Stream function and tries to uplink the commands and the Count_Transmission_Number function counts the number of times a command was uplinked. When an Uplink_Failure occurs, the Retransmission_Command flag is checked; if this flag is set to Enable and if the Transmission_Limit is not reached then Command is retransmitted. The operator can set the Transmission_Limit to a suitable number of retransmissions based on the uplink conditions.

The Spacecraft function receives the Spacecraft_Uplink_Data and produces as output Command_Transmission_Status and Telemetry_Data. The code segment for this transition consumes the tokens provided for the spacecraft through transmission and produces Command_Transmission_Status, Telemetry_Data and Telemetry_Data_Arrived tokens to be used by the Receive_Command_Status_Data function. The uplink_status of the command is chosen randomly according to a predetermined probability. The probability distribution can be changed according to the desired scenario.

The Receive_Command_Status_Data function receives the Command_Transmission_Status and Telemetry_Data, and notifies the ICC (Instrument_Control_Centers) about the Command_Status. The code segment associated with this transition produces the Spacecraft_Command_Status_Data token to be used by the Evaluate_Spacecraft_Command_Status function.

The Evaluate_Spacecraft_Command_Status function process the Spacecraft_Command_Status_Data and checks the uplink_status and execution_status of the command uplinked. It passes this information to the EOC_Controller; if there was an uplink failure a retransmission of the command will be attempted, provided the retransmission_command flag is set and the transmission limit has not been reached. The operator is notified whether the Command is transmitted or not. 
45 ‘ iclr=FALSE,oc=\{cn=0,com=GOOD,a=TRUE,c=NON_CRITICAL,t=0\},cli=TRUE $\}$ $100^{*}\{\mathrm{cn}=0, \mathrm{com}=\mathrm{GOOD}, \mathrm{a}=\mathrm{TRUE}, \mathrm{c}=\mathrm{NON}-\mathrm{CRITICAL}, \mathrm{t}=0\}$

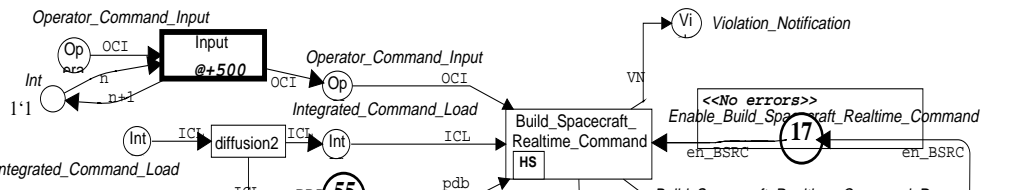

EOC_Commanding

100“TRUE

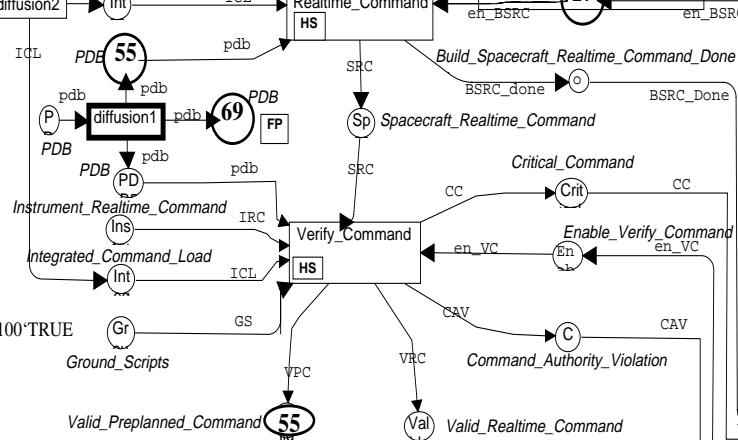

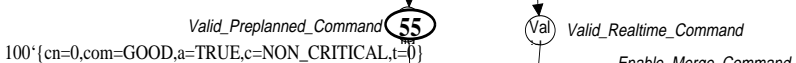

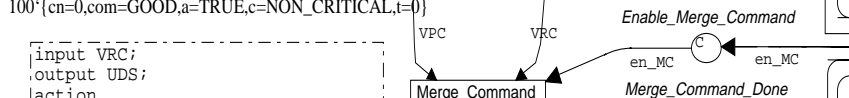

laction

val $c \_n=\# c n$ VRC;

ival i_t $\mathrm{t}=\# \mathrm{t}$ VRC;
ival $\mathrm{u}_{\mathrm{t}} \mathrm{t}=\mathrm{time}() ;$

val $U_{-} D_{-} S=\left\{c n=c_{-} n\right.$, it $=1 \_t$,

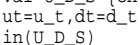

lin
iendi

$$
\text { Enable_Transmission_Command }
$$

27 en en_TC

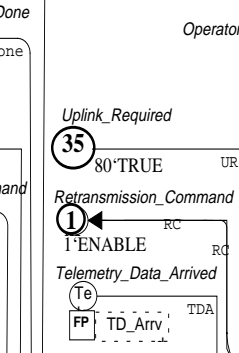

Operator_Command_Request
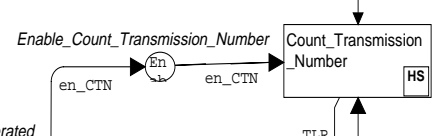

Tine

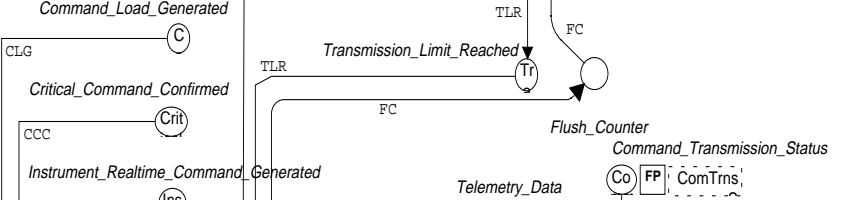

IRCG
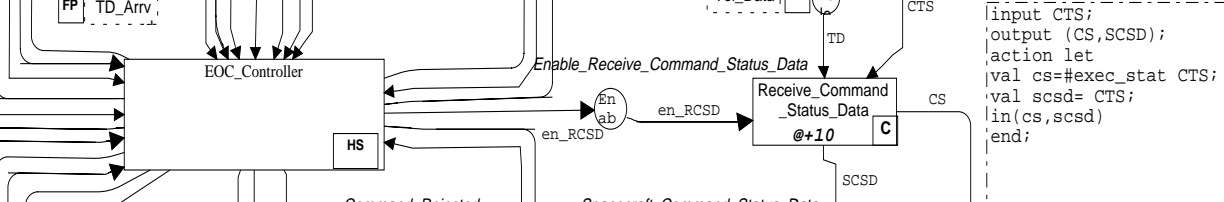

45
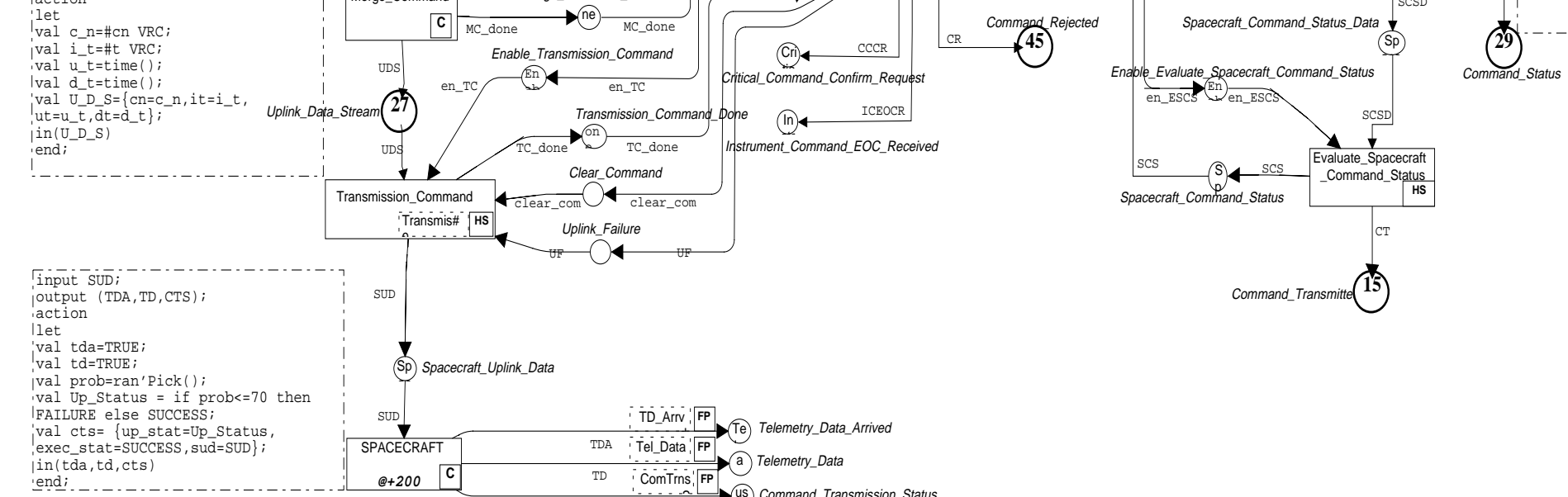

Command_Transmitte 15

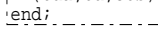

SUD

7

Sp) Spacecraft_Uplink_Data

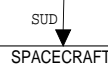

e+200 C

$\underset{1}{\sim}$

(a) Telemetry_Data

Figure 6-6 Page 2 of CPN model (commanding) 
The EOC_Controller function is mapped from the Controller on the Commanding DFD. This sub module is specified in detail on a separate CPN page (Figure 6-6) and contains the control specifications for the Commanding module as a whole. It produces the tokens necessary for the invocation of the functions of the Commanding module and to send messages to the operator.

The initial state of the system is specified in the initial markings for the input places. The initial marking of an input place represents the number of input tokens present in that place in the initial state. For example, in Figure 6-6 the initial marking for the place Operator_Command_Input is specified as 45 tokens of the type Operator_Command_Input with duly assigned values like Index_Command_Load_Request as False, the Operator_Command is NON_CRITICAL, etc. The transition Operator_Command_Input has been added to supply the operator_command_input tokens to the BSRC function at a desired rate. The time taken for a function can be represented in the time region for each transition. For example, Figure 6-6, shows the Operator_Command_Input transition takes 500 time units and the Receive_Command_Status_Data function takes 10 time units. The Integrated_Command_Load tokens and the Project_Database tokens need to be supplied for different transitions and hence two extra transitions have been added to generate multiple copies of these tokens to feed different transitions. The code segments of different transitions are updated to implement the desired scenario. For example, the probability for failure in transmission has been set to 70 percent in the code segment of the transition representing the Spacecraft stimulus (Figure 6-6).

The initial marking of the place START is specified as 1'TOKEN, enabling the transition T1 when the simulation is started. When T1 fires it invokes the function init() as specified in the code segment for $\mathrm{T} 1$. The function init() is written in a temporary declaration node and initializes some global CPN variables used across the different CPN pages and the statistical variables needed for the dynamic analysis. For example, the number of commands transmitted is initialized to zero, a statistical variable for the total_uplink_time is created and initialized with a value of 
zero, etc.

There are eight possible states for the Commanding module:

- Wait_Command,

- Build_Realtime_Command,

- Verify_Command,

- Wait_Confirm, Merge_Command,

- Uplink_Command,

- Wait_Telemetry,

- Evaluate_Command_Status.

When the transition T1 fires, the Controller goes to the state Wait_Command and waits until one of the tokens Operator_ Command_ Request, Instrument_ Realtime_ Command_ Generated or Command_Load_Generated arrives.

When an Operator_Command_Request token is received by the Controller, it goes to the state Build_Realtime_Command and enables the BSRC module by supplying the token Enable_Build_Spacecraft_Realtime_Command (transition T9 is fired). When the token Build_Spacecraft_Realtime_Command_Done is received from BSRC, the Controller enables the Verify_Command function and goes to the Verify_Command state (transition T10 is fired).

If the Controller received an Instrument_Realtime_Command_Generated token or a Command_Load_Generated token from the ICC (Instrument Control Centers) while in the Wait_Command state, it enables the Verify_Command function and goes to the Verify_Command 
state (Transition T2 or transition T17 fires).

When the Verify_Command function checks the authority of the issuer of the command and verifies whether the command is Critical or Non_Critical, the Controller proceeds either to the Merge_Command state or Wait_Confirm state or goes back to the Wait_Command state. If the Command_Authority_Violation (CAV) was false and the Command was Non_Critical, the Controller enables the Merge_Command function and goes to the Merge_Command state (transition T4 fires). If the Command_Authority_Violation was true, then the Controller goes back to the Wait_Command state and waits for a new command discarding the previous command (transition T3 fires). If the Command is Critical, it informs the operator by generating a Critical_Command_Confirm_Request token (transition T5 fires) and upon receipt of a positive response from the operator goes to the Merge_Command state (transition T7 fires). If the operator wished to discard the command by setting the Critical_Command_Confirmed token to DISCARD, then the command is discarded and the Controller goes to the Wait_Command for the processing of the next command (transition T8 fires).

When Merge_Command function is done and the Uplink_Required token is set to true, the Controller goes to the Uplink_Command state (transition T11 fires). If the Uplink_Required token is false, the Controller goes back to the Wait_Command state (transition T6 fires). When transition T11 fires and the Next_Command token is set to true, the Controller enables the Transmission_Command function to uplink the next command (transition En_Trn1 or transition En_Trn2 fires). In the initial state one token of Next_Command is supplied and is set to true, that will be used by the first command to be uplinked. When the uplinking of a command is done, the Next_Command token is set to true so the next command can be uplinked (Transition T13 puts a token in the Next_Command place). When a command is being uplinked the Controller enables the Count_Transmission_Number function by setting the Enable_Count_Transmission_Number token to true (transition New_Com fires).

When the Transmission_Command function is done, the Controller goes to the 


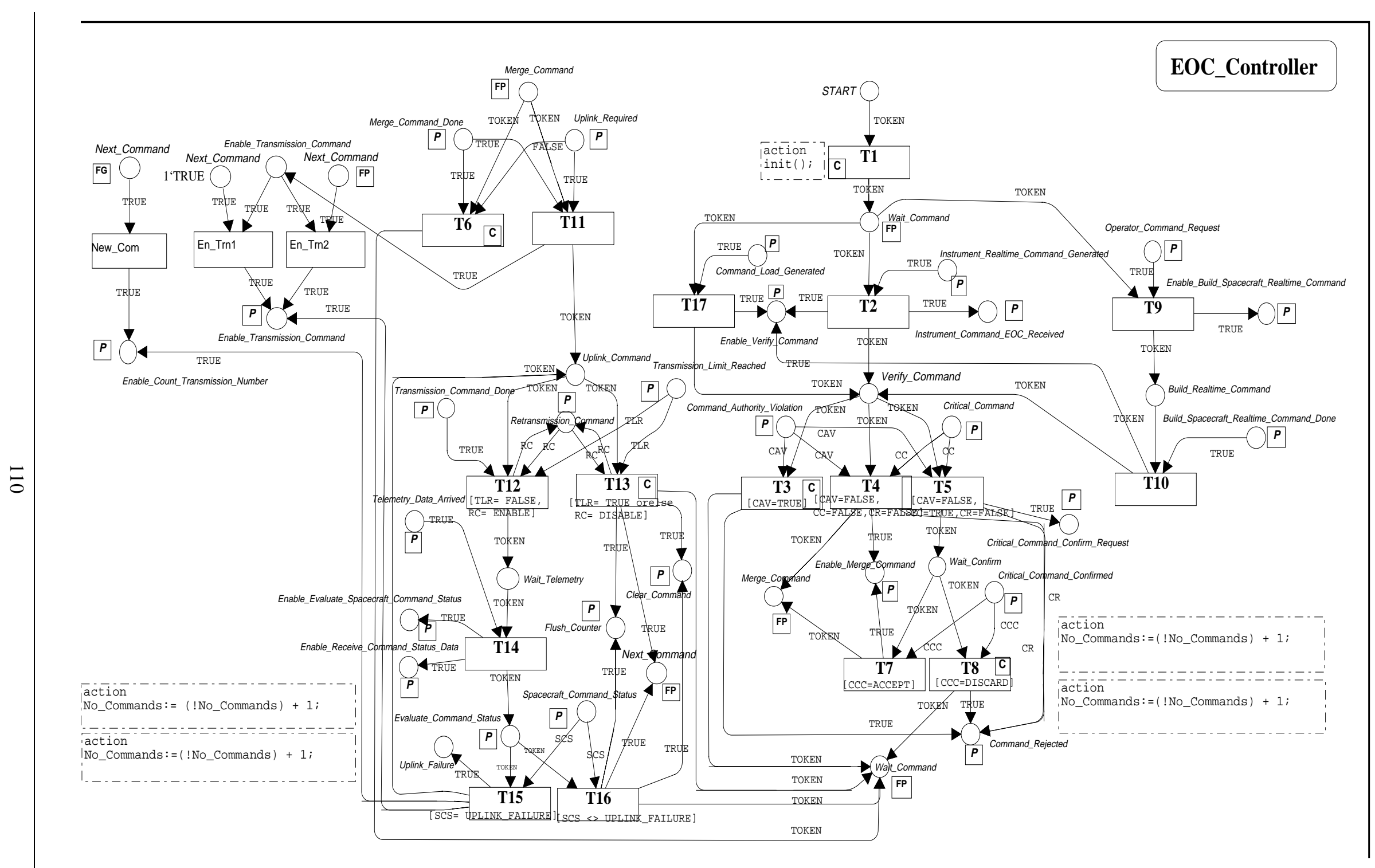

Figure 6-7 Page 9 of CPN model mapped from C-Spec of commanding 
Wait_Telemetry state (transition T12 fires). The transmission command function can be fired for retransmitting a command when an uplink failure occurs. The retransmission is done only when the Retransmission_Command token is set to Enable and when the Transmission_Limit_Reached is true. When Transmission_Limit_Reached is True or the Retransmission_Command is set to Disable, the command is discarded and the Controller goes to the Wait_Command state (transition T13 fires). When T13 is fired it puts a token in Next_Command, Flush_Command and Clear_Command so the command buffer holding the current command is cleared, the counter is reset to zero and the uplinking of the next command is possible.

While in the Wait_Telemetry state, when the Telemetry_Data_Arrived signal is received from the spacecraft, the Controller goes to the Evaluate_Command_Status state enabling the Receive_Command_Status_Data function and the Evaluate_Spacecraft_Command_Status functions (transition T14 fires).

When the Controller is in the Evaluate_Command_Status state and the Spacecraft_Command_Status token received from Evaluate_Spacecraft_Command_Status function is set to Uplink Failure, a retransmission of the command is attempted by enabling the Transmission_Command function; the Controller goes to the Uplink_Command state (transition T15 fires). The operator is also notified of the Uplink Failure by setting the Uplink_Failure token to true. If there was no failure, the Controller goes back to the Wait_Command state to process the next command (transition T16 fires). When transition T16 fires it produces the next_command, flush counter and clear_command tokens similar to the transition T13 (as explained above).

The initial marking for the place START is 1'TOKEN and for the place Next_Command is one token with value "TRUE". The code segment for the transition T1 calls the function init(), that initializes the CPN variables needed for accumulation of statistics as explained earlier. The transitions T3, T6, T8 and T13 have code segments to update the number of commands processed when the command has not been uplinked. When a command is successfully uplinked, a transition in the function Evaluate_Spacecraft_Command_Status updates the number of 
Commands processed and the number of Commands uplinked and collects the statistical data for the uplinked command.

During the process of building the CPN model for Commanding, several ambiguities/ inconsistencies were found in the Teamwork model of Commanding (Figure 6-3 and Figure 6-6):

- The implementation of the Project Database (PDB) in the model of Commanding is inconsistent with NASA requirement specifications [53]. According to NASA specifications there should be a provision for the operator to set the Retransmission Command flag as desired. This flag is checked before retransmission of a command when an uplink failure occurs. The inconsistency in Teamwork might be due to the ambiguously defined requirement specifications that do not explain clearly where this capability is implemented.

- The Retransmission Command function in the Commanding model buffers the command previously uplinked and if there was an uplink failure it tries to retransmit the command. NASA requirements [54] specify EOC shall implement retransmission such that all commands transmitted since the last command known to be received and accepted at the spacecraft shall be retransmitted in the same order as originally transmitted. If the model is executed in a pipelined design as explained in [43], it is easy to notice an inconsistency in the model of Commanding related to the above requirement. If the command was successfully uplinked, but could not be executed by the spacecraft, the operator is notified. The commands waiting to be uplinked will be uplinked immediately (see the transition from the state at the bottom right of the state diagram in Figure 6-6). The functionality of retransmission as specified in the NASA requirement specifications depends on the successful uplinking and execution of the buffered commands on the spacecraft and the results of their execution. According to the specifications, it appears that if a command is uplinked then the commands following it are uplinked without waiting for an execution status. When a command could not be executed by the spacecraft, all the commands 
uplinked after it should be retransmitted in the same order. The functionality is not clearly described in the specifications and it is not specified how the uplinked commands are buffered and when they are executed. This might be the cause for the incorrect representation in the Teamwork model of Commanding, that was translated into a CPN model.

- The control flow from Build_Spacecraft_Realtime_Command to Verfiy_Command (Figure 6-6) does not specify the condition of transition. This might allow the invocation of Verify_Command process before the Realtime Command is built.

- In the Build_Spacecraft_Realtime_Command component, the function Validate Command validates the realtime command and generates a Violation_Notification signal if a realtime violation is detected. But this signal is not communicated to the EOC Controller so further processing of that command is stopped.

- The condition for the transition from Merge_Command process to Wait_Command process (Figure 6-6) is not specified. This might result in improper uplinking of commands.

- The transition from Uplink_Command to Wait_Telemetry (Figure 6-6) is not consistent with NASA specifications. The Wait_Telemetry process should be invoked only after the uplinking of the Command is completed.

- The Wait_Telemetry process is enabling the Count_Transmission_Number (CTN) function. This is not necessary. The Count_Transmission_Number function has already been invoked in the previous state that counts the transmission and reports to the Transmission_Command function whether the Transmission Limit has reached or not. If CTN is invoked again it counts the command again, that is incorrect.

- When Uplink_Failure occurs, we go back to the Uplink_Command state from the 
Evaluate_Spacecraft_Command_Status state (Figure 6-6). We check the transmission Limit and the Retransmission_Command flag, then perform a retransmission of the command. These conditions are checked in the Evaluate_Spacecraft_Command_Status state and if a retransmission is required then Transmission Command function should be invoked, otherwise the system should go to the Wait_Command state.

- The Command_Rejected signal is used in Evaluate_Spacecraft_Command_Status state, that is inconsistent because if Command_Rejected was true then the Evaluate_Spacecraft_Command_Status function should not have been invoked.

Using the dynamic models developed so far, the following sections describe two types of dynamic analysis performed on the commanding component.

\subsection{Dynamic Analysis of the Commanding Component}

Using the framework developed as part of this work, dynamic analysis of the commanding component were developed. These models were used to perform two types of analysis as given below:

- Risk Assessment by Ammar et. al in [44], [55] and [56].

- Performance and performability analysis by Mogulothu et. al in [43].

The risk assessment is based on the results obtained from the three types of analysis: Complexity Analysis, Severity Analysis, and Heuristic Risk Analysis. Complexity analysis is further based on four aspects of software system complexity. These are termed as Static, Functional, Operational and Concurrency complexities. Metrics for the last three types of complexities are generated using scenario based simulation of the dynamic behavior of the commanding component.

Functional complexity is determined by analyzing sequential and the pipeline model 
running the same scenario. The probability of each transition being executed during the simulation is calculated. Once these probabilities are determined, the functional complexity of the two models [44]. Operational complexity measure indicates the cyclomatic complexity of the subgraph being executed during the simulation. Concurrency complexity measures the number of processes running at the same time. For example, the simulation shows that in the pipeline model, there are more processes running at the same time compared to the sequential model.

For severity measurements, CPN model of the systems are used to study the effects of the failures on a component-by-component basis in the two systems. Using CPN model as a tool for failure mode and effect analysis, faults (one at a time) are injected in to the components of the systems. These simulation are used to study the effects of the failures. Severity numbers are then assigned to each class of severity based on the severity and criticality of their failures on the system. In the final step the figures calculated for complexity and severity of the components in the system, are used to compute the heuristic risk factor as the final stage in the risk assessment procedure.

Performance and performability analysis was performed by Mogulothu et. al in [43]. More details of this analysis are given below:

\subsection{Performance Analysis of the Commanding Component}

This section discusses the performance analysis carried out on the Commanding component of the EOS system and presents the results and conclusions of the analysis performed by Vinay Mogulothu.

Two scenarios, i.e., sequential and pipelined scenarios were simulated to assess the performance and/or performability of different execution profiles of the Commanding model. 


\subsubsection{Performance under normal sequential execution}

The Commanding model was simulated to analyze the performance under favorable conditions. The timing behavior of each module was specified and it was assumed that all the modules function normally. The Operator_Command_Input which is provided as an initial token activates the Build_Spacecraft_Realtime_Command (BSRC) function where the command is successfully built and validated. The Verify_Command function then verifies the authority of the command and produces a Valid_Realtime_Command. This is merged with a Valid_Preplanned_Command obtained from the Integrated_Command_Load input. The merged commands form an Uplink_Data_Stream which is uplinked to the space craft through the successful execution of the Transmission_Command function. The status data from the space craft is received and evaluated to indicate a successful reception as the Spacecraft_Command_Status. This in turn is used by the EOC_Controller to activate BSRC to build and validate the next Operator_Command_Input. The simulation of this scenario produces measures on the throughput and total execution time of the operator commands. The average response time for a command and the average channel utilization time are also calculated. The average channel utilization time was almost equal to the average response time (Figure 6-11). This indicates that the bottleneck in the system is the communication channel. This is because the time taken to build, validate \& verify a command is comparably less than the time taken for uplinking of the command and downlinking of the command_status. The total execution time for a sequence of 45 operator input commands was more than the sum total of the response times for these commands because of the time taken for the input of the commands. The response time per

command was constant, since each command encounters the same conditions. The behavior of the system can be observed to be the same for the total simulation period. (Figure 6-8).

\subsubsection{Performance under pipelined normal execution}

In this case instead of processing one command at a time, a sequence of operator 
commands are pipelined through the system. Several functions are concurrently active to process the command sequence.

The flexibility of Colored Petri Net notation to express the control flow of a system greatly eases the analyst's efforts to design alternate specifications and explore the system behavior under such specifications. The analyst may need to make minor modifications to come up with a different specification of the system. The State Transition Diagram specification of TeamWork is limited in the sense that it does not allow the specification of a parallel design without the introduction of many more states and transitions making the system too complex to visualize and analyze. The specification of the pipeline design in the Design/CPN model is almost identical to the sequential design (Figure 6-10). The only page that was modified was the controller for EOC Commanding.

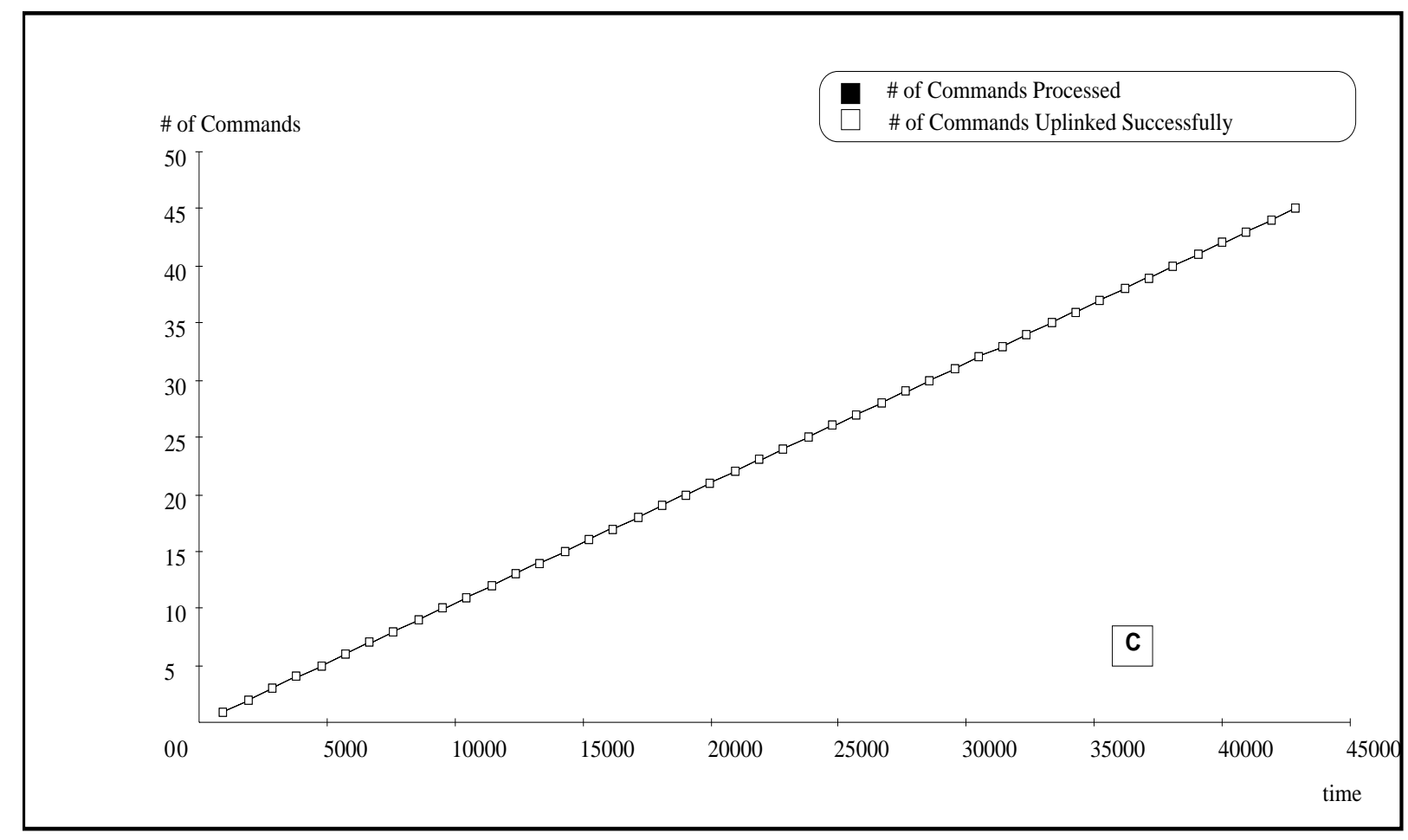

Figure 6-8 Scenario 1 : Sequential Execution under Favorable Conditions 
The minor modification that was done in the EOC controller is an addition of an arc from the Transition T-10 to the State Wait_Command. This leads to the system being executed with

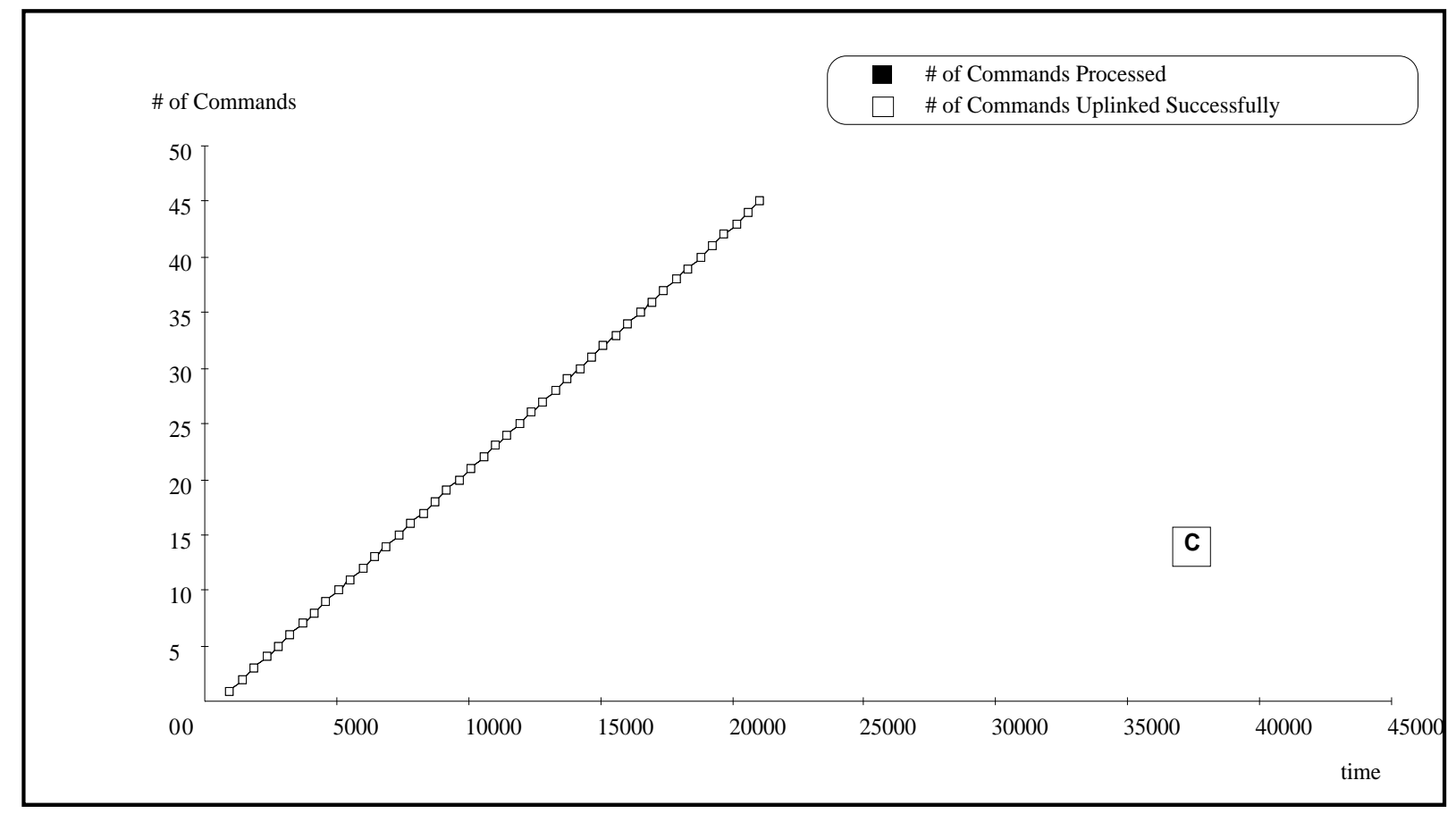

Figure 6-9 Scenario 2 : Pipelined execution under favorable conditions

several different "states" being simultaneously active. This is because the transition T-10 fires when the Build_Spacecraft_Realtime_Command function processes the Operator_Command_Input and the Verify_Command module is activated. In the sequential scenario the Verify_Command would process the output of Build_Spacecraft_Realtime_Command, while all other processes are inactive and the next Operator_Command_Input will be processed only after the current command is transmitted and acknowledged of the transmission. In the pipeline design the firing of transition $\mathrm{T}-10$ deposits a token in the Wait_Command state as well as Verify_Command state. This enables the Build_Spacecraft_Realtime_Command to process the next Operator_Command_Input if any were present even while the previous command is still being processed. This is propagated to all other processes down the line, since each process would be activated by the presence of the tokens 

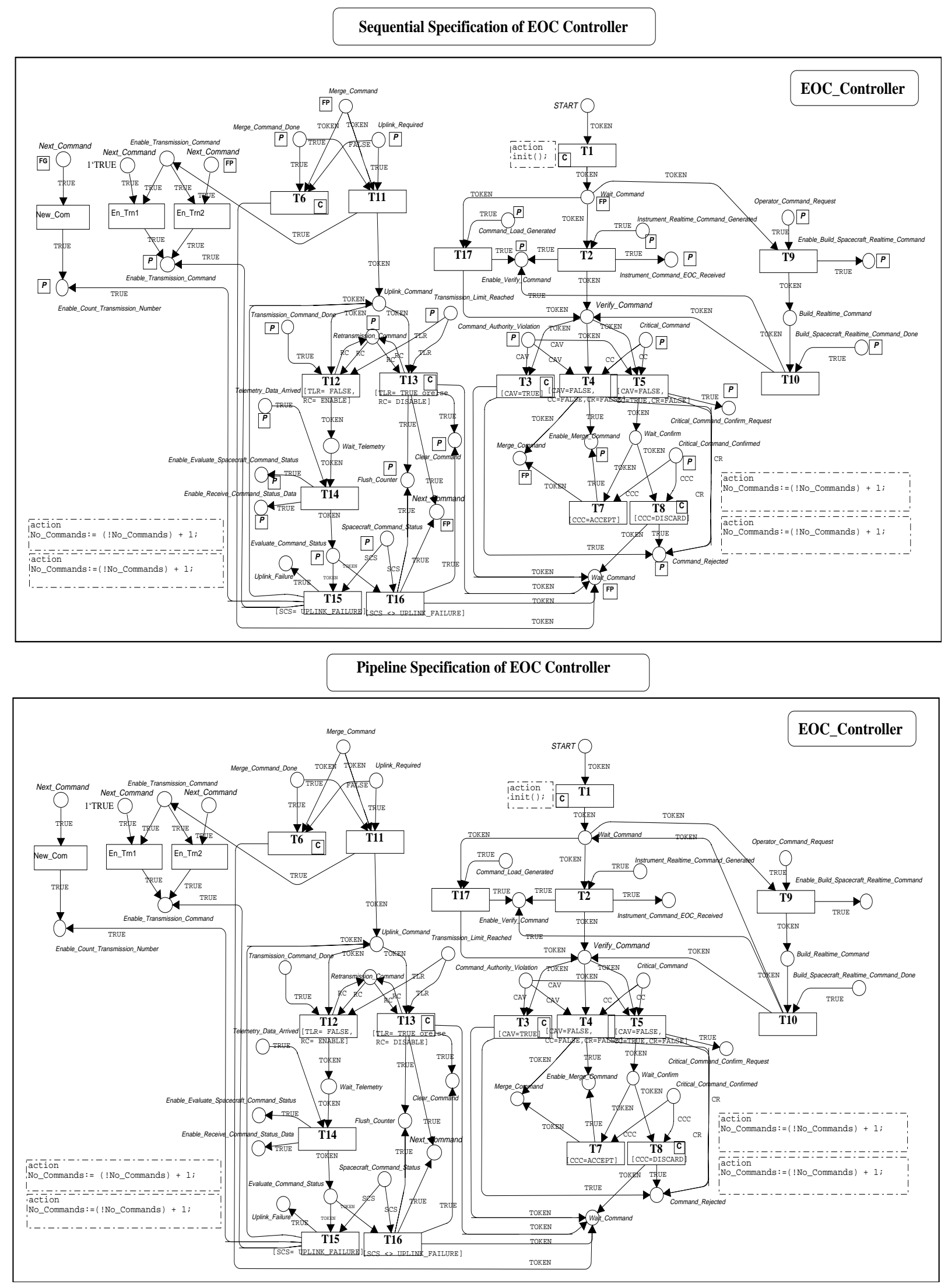

Figure 6-10 Sequential \& Pipelined Specification of the EOC Controller 
in the corresponding states and the input places, which would be supplied by the successful completion of the previous state. Thus a pipelined execution of the system is effected. Such a specification of the system being present in multiple states at the same time is not possible in the TeamWork specification of the model. This gives the analyst more power to explore several designs to gain valuable insight into the behavior of the system under such designs and also evaluate possible advantages or disadvantages of such specifications. With this insight the analyst could suggest an alternate specification which helps the performance of the system and/or a less complex design, which necessarily implies less risk in the system.

The performance improvement under this pipelined design is observed. The throughput of commands for the pipelined execution is double the throughput for sequential execution under the same conditions. This is evident from the slopes of the curves in Figure 6-8 and Figure 6-9. The average response time is only slightly greater than the sequential case. The response time per command was constant throughout the execution of the simulation. This is because the input rate of commands is low considering the time taken for the operator to input the command. Thus the average response time for the pipelined case is almost equal to that of the sequential case (Figure 6-11), but if a sequence of preplanned realtime commands is read from a script of commands then the average response time of a command will be much larger than the sequential case. This is because the input rate would be higher and there will be a build up at the communication channel. The commands have to wait for longer times at the channel for being uplinked. This is an added risk in the pipelined design since some of the commands will have a large waiting time and consequently a large response time. These commands may violate the deadlines of uplinking and execution because of this delay. If the rate of input is higher then the communication channel will be a major bottleneck in the system, since the commands get buffered at the channel waiting to get uplinked. The channel utilization time per command is the same as in the sequential simulation (Figure 6-11). 


\subsubsection{Performability analysis of the pipelined design}

In this case, the commanding model was simulated with faulty behavior in the system. This was accomplished by simulating the effects of failure and recovery in the system functions such as BSRC and Verify_Command. The failure and recovery activities of these modules were simulated by adding CPN ML code in the code segments associated with the respective transitions to simulate the activity of causing a failure with which an estimated recovery time is attached. The commands that need to get processed by this module should wait until the module recovers from the failure and hence the pipeline stalls, i.e. the modules down the line would receive no more new input from the failed module. The degraded performance of the system under failures and repairs is observed in the simulation. This scenario is simulated for a sequential design of the system and also for a pipelined design and the relative performance is evaluated.

The throughput of commands for the pipelined execution under failures of the system modules is again larger when compared to the throughput for sequential execution under the same conditions (Figure 6-13 and Figure 6-14). This indicates that the pipelined design has improved the system performance under faulty conditions also. However, the throughput of the pipelined model should reduce when commands are flushed from the pipeline due to an uplink or execution failure. The response time per command in both pipeline and sequential execution is greater than (almost 2.5 times) the channel utilization time per command. When there are failures in the communication channel only, and all the modules of the system are functioning properly, the communication channel would be a major bottleneck in the system. In such a case, the response time per command would almost be equal to the channel utilization time per command. This is because of the uplink failures and retransmission of commands which increase the time spent in uplinking of the commands. When there are failures in the system modules then the commands experience a delay in processing and there will be a decrease in the build up at the communication channel. Under these circumstances the response time per command is greater than the channel 


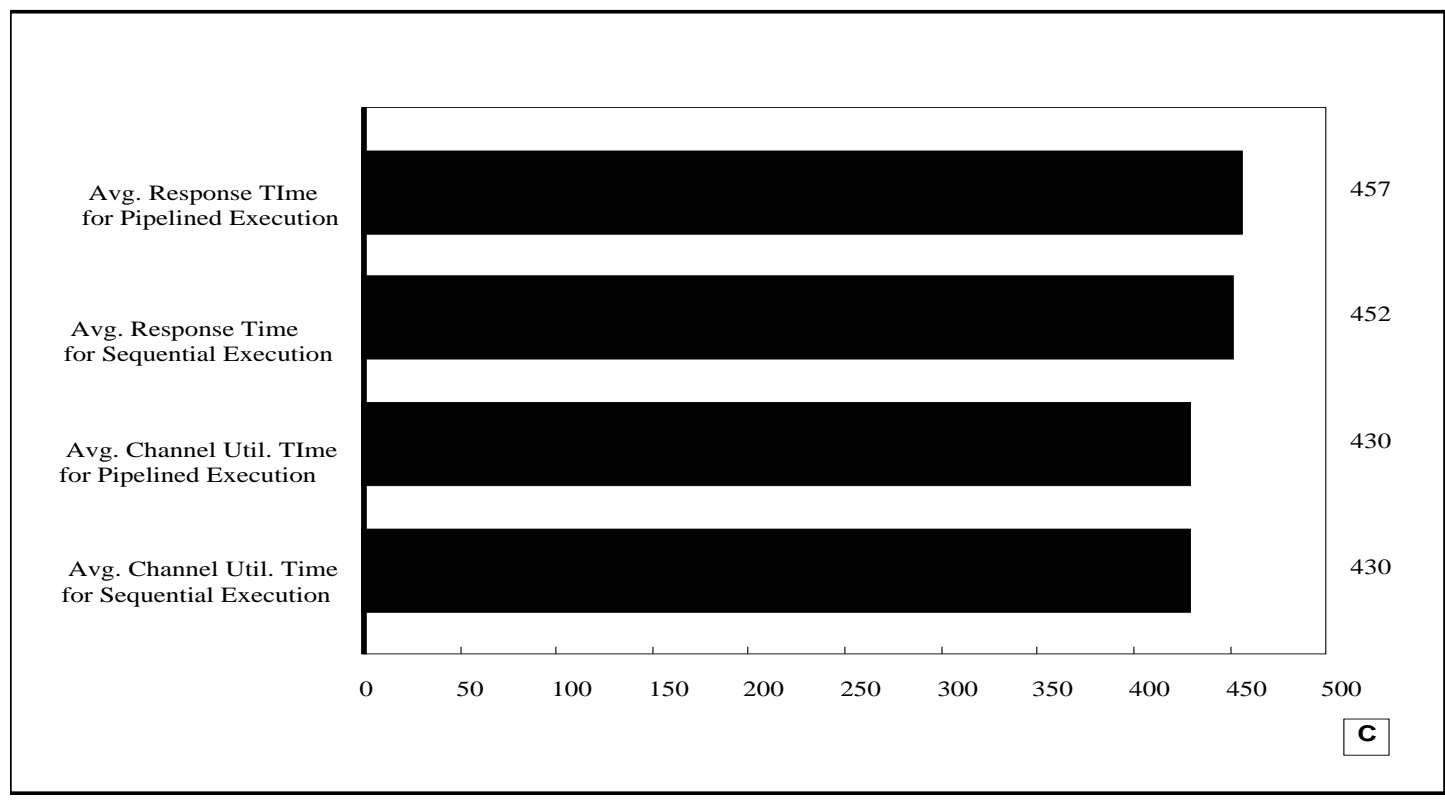

Scenario 1 : Sequential execution under favorable conditions Scenario 2 : Pipelined execution under favorable conditions

Figure 6-11 Response time \& Channel utilization time per command for Sequential \& Pipelined execution under favorable conditions

utilization time per command. This is because when the faulty modules like BSRC or Verify_command fail and the system takes some time to recover to normal execution, the communication channel can uplink the commands which were already processed and verified.

It is also observed that the processing time for each command is not the same throughout and that some of the commands are lost without being uplinked. This is because of the faulty behavior in the system and the communication channel failures. Whenever there is a loss of command we can observe this by the widening of the gap between the lines representing the total_commands_processsed and total_commands_uplinked (Figure 6-13 and Figure 6-14). When a command is not successfully uplinked the transmission command module retransmits the same command (for a maximum of 3 tries) and if the command is not successfully uplinked within three chances then the operator is notified of the uplink failure. This faulty behavior also leads to the difference in response times for each command. 


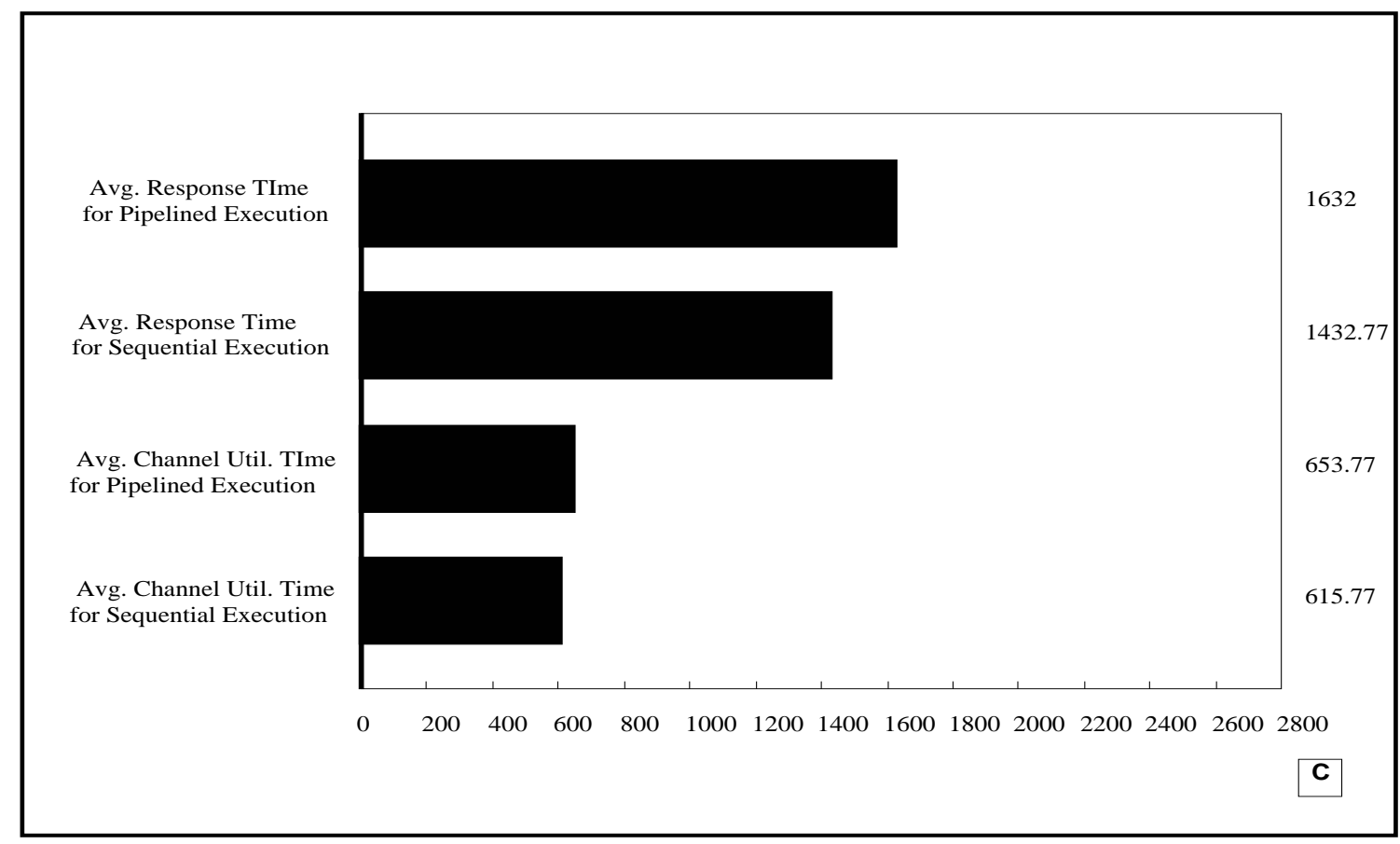

Sequential Execution under Failures in Communication and Fault Injection Pipelined Execution under Failures in Communication and Fault Injection

Figure 6-12 Response time \& Channel utilization time per command for Sequential \&

Pipelined execution under failures 


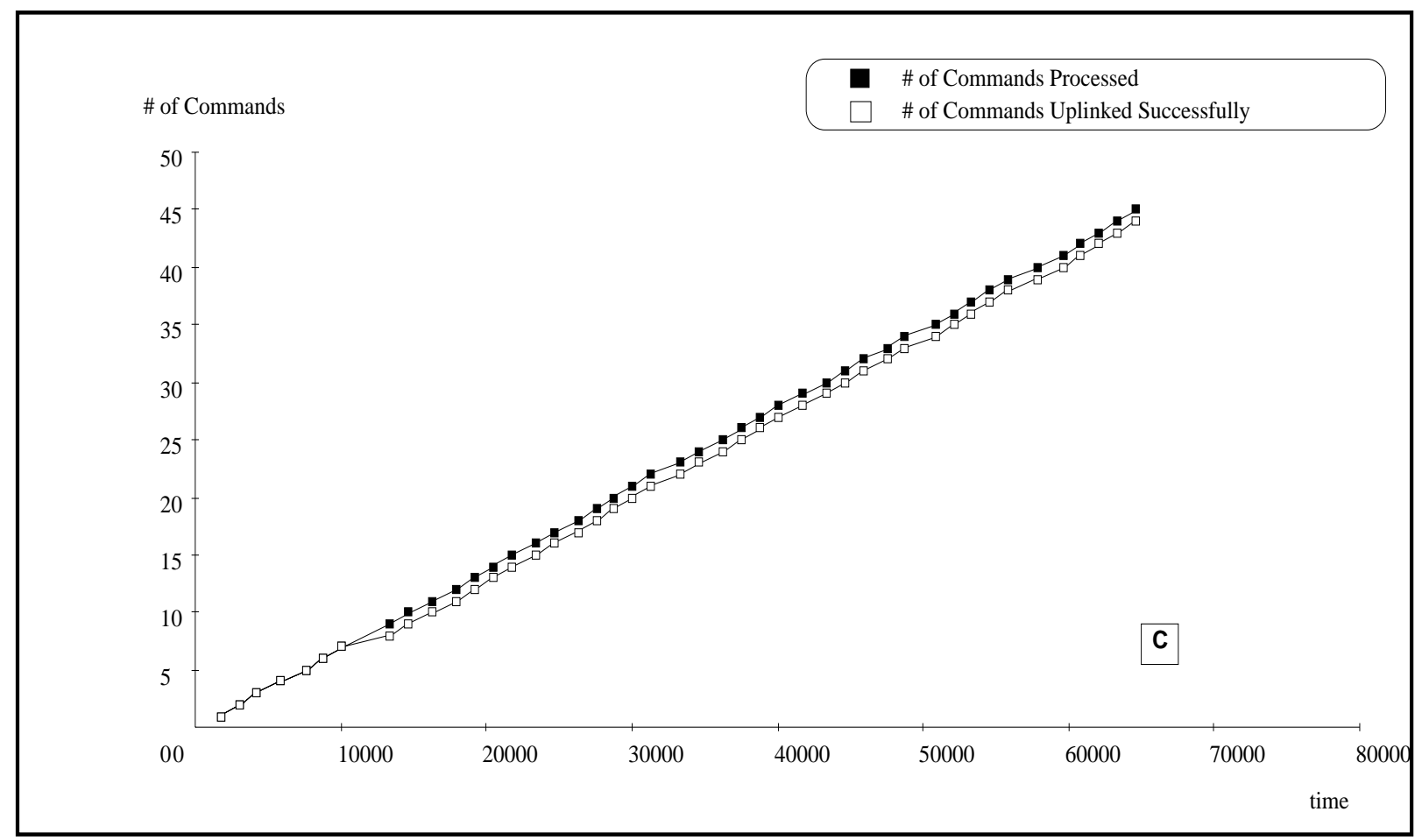

Figure 6-13 Throughput of Commands for

Scenario 5 : Sequential Execution under Failures in Communication and Fault Injection

The average response time (the response time per command) for pipelined execution is greater than that of sequential case because the commands which are being processed by the 


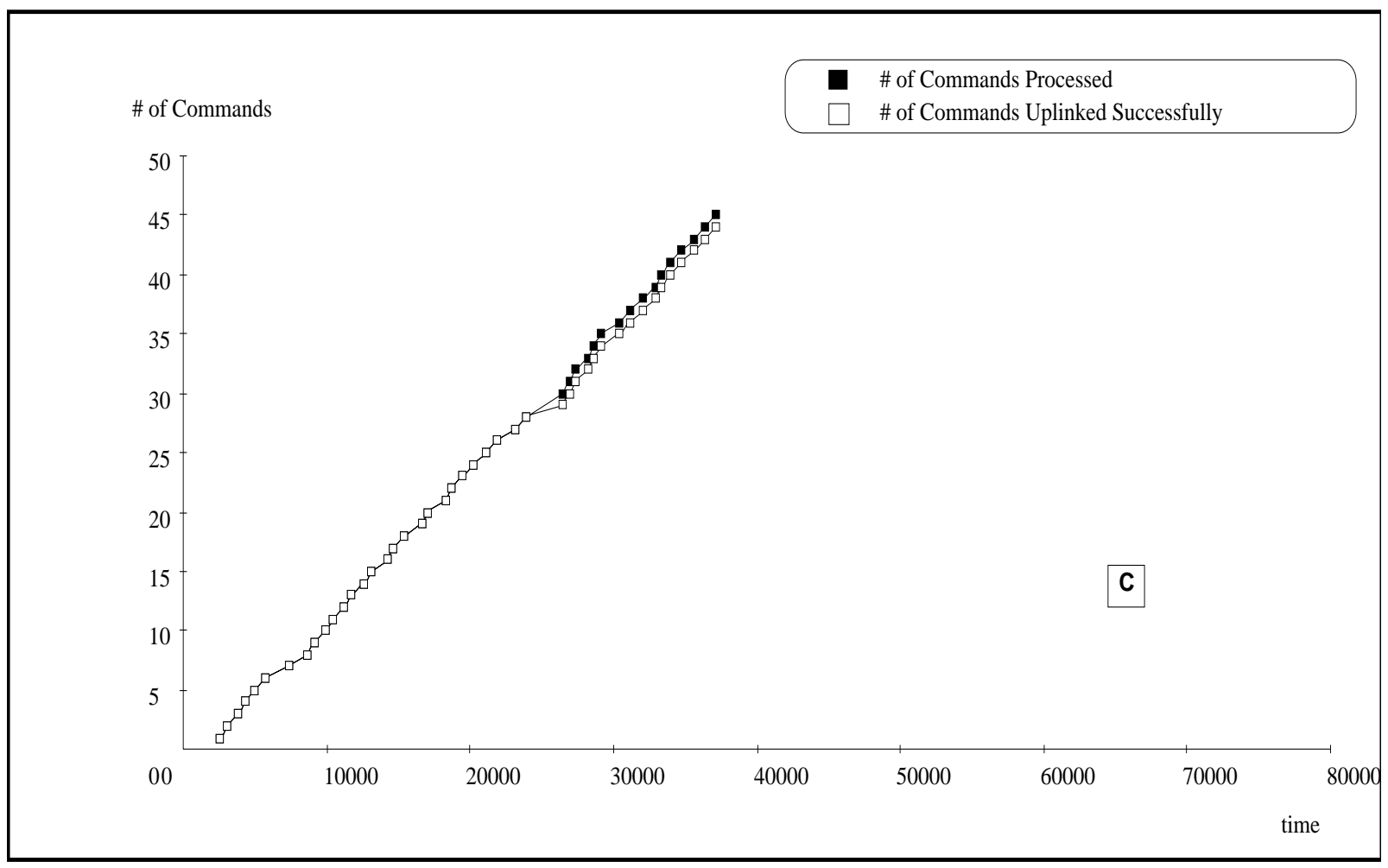

Figure 6-14 Throughput of Commands for Pipelined Execution under Failures in Communication and Fault Injection

initial modules still have to wait to be uplinked when a command being transmitted experiences an uplink_failure and is being retransmitted. Although this difference is not much (Figure 6-12), for a particular command the pipelined case might take a lot longer to uplink the command due to failures in system modules and uplink failures of the previous commands as well as itself. Also when realtime commands are read from a preplanned script then the rate of input commands would be greater and this would make the commands wait longer than usual in the pipeline case. This also adds to the risk involved for the pipelined execution. Since under real time conditions a command might miss the deadline for uplinking which may result in hazardous conditions. This needs to be taken care of in the system design.

\subsection{Summary}

This chapter presented a process to addresses the dynamic analysis problem for a large 
realtime system early in the development. Requirements analysis models for this systems were available from the developers in SART notation. A five step process was used to generate the dynamic simulations for the specifications. The dynamic simulations were used to perform performability analysis and risk assessment for different scenarios. 


\section{Chapter 7 Summary, Conclusions, and Future Work}

This chapter presents a summary and conclusions of this dissertation, and several areas of future work.

\subsection{Summary and Conclusions}

This work addresses the dynamic analysis problem for two types of notations used for software specifications: SART and UML. The objectives are to develop methods to map these artifacts into formal dynamic specification models. Once the process is in place, the proposed method was implemented for a component of a large scale software system.

A five step process was developed for generating CPN models from SART models. Based on the mapping rules proposed in Chapter 4, a translation utility was developed to automate the mapping process. The process preserves the hierarchy in the original specifications and hence it is applicable to large complex specifications. The proposed process of developing dynamic CPN specification models is a non-trivial process. An automated tool environment is needed to support the steps of this process as discussed in the next section.

CPN models generated using our framework resemble the SART model in their hierarchy and layout (Figure 4-2, Figure 4-3). This makes it easy for the analyst to readily identify SART units that may need modifications in light of the dynamic analysis. Another contribution of this work is that the CPN models generated from separate SART models can be combined together ( Section 4.9) for simulating the integration process of different modules. This helps to perform dynamic analysis of interfaces between different modules.

The large scale case study used in this work is based on EOS (Earth Observation System), also known as "Mission to Planet Earth". This system will be deployed by NASA. The core of EOS is a real time component called FOS (Flight Operation Segment). Chapter 6 presented the 
dynamic models of a component of FOS generated using this unique process.

While the first problem addressed by this work is related to SART, the second problem addressed by this work was to develop a framework for dynamic analysis of UML models. This aspect of research was more challenging due to the presence of four types of UML diagrams used for analyzing requirements specification. The UML diagrams capture related yet very different types of information. One such example is the class diagram that gathers class related information. This information is quite different from the information present in the use-cases where the functional perspective is captured. This problem was addressed in this work by combining two well known methods in the field of software engineering. The first is, Nissen and Jarke's [27] metamodeling concept and the second is the stimulus-response method proposed by Alan Davis [51].

The proposed process of generating DMMs from a given set of UML diagrams was presented in Chapter 5. The five step mapping process combines semantics information on the dynamic behavior from UML models and generates a DMM. The resultant DMM can be mapped to different dynamic simulation tools such as: ROOM (realtime object oriented modeling) or Design/CPN. As a proof of concept, DMMs are generated for a scenario from a pacemaker example. Later the information contained in DMM was used to create CPN models.

This work contributes to the state of the art in software engineering by providing a framework for generating dynamic models. Our research group has used this framework to perform different types of analysis at the early phases of software development. Dynamic analysis were performed using the dynamic models presented in Chapter 6. Performability analysis was performed for a large scale realtime system by Mogulothu et. al in [43]. Ammar et. al used this framework to perform risk assessment at the early phases of software development in [44], [55] and [56]. Cukic et. al. in [45] used this framework to identify high risk scenarios.

In summary the contributions based on the work presented in this dissertation are as 
follows:

- A framework for generating dynamic models from SART specification models.

- Application of this framework on a component of a large scale software system.

- A framework for generating dynamic models from UML specifications models.

The following section describes potential future work on the basis of this dissertation.

\subsection{Future work}

Potential future work can include many facets of software development as well as verification. Most of the suggested areas for future research focus on the analysis of software specifications early in the development lifecycle. These areas may include new metrics for measurement of dynamic behavior, new tools to process the information related to dynamic behavior, and the use of dynamic analysis in the risk assessment based applications. The following paragraphs describe these areas in more detail.

\subsubsection{Tool support for generating dynamic models}

The proposed process of translating specifications from developers' models to dynamic models is non-trivial. There is a need for developing collaborative environment that contains interfaces to different CASE tools, interfaces to the dynamic analysis tools and the utilities for translating semantics information between different tools. This is due to the fact that a model based on rigorous notation such as CPN requires much effort to be completed. The original models based on SART or UML may include many ambiguities complicating the mapping process. Semantics mapping is a nontrivial process that needs to be abstracted, modeled and supported by an environment of integrated tools. For SART, a Semantic Transfer Utility (STU) was used to transfer the structure of the analysis models supplied by the developer. As STU deals with SART based models only, there is a need to develop similar translation utility for UML based 
specifications.

MATRIXx is a realtime software specification tool, being used in aerospace industry for generating specifications for embedded systems. This tool uses a modeling notation that is very similar to SART. STD is used for control specifications in MATRIXx similar to STD used in the SART as well. Parent process of SART is similar to the superblock used in MATRIXx. Data flow representation is very similar in both the tools. Dynamic analysis based on the framework presented in this dissertation can in future be used for MATRIXx models.

Another area of future work in tool support, is to integrate dynamic analysis techniques for specifications based on the ROOM notation mapped from UML specifications. In this dissertation Design/CPN was used as an example tool that can provide dynamic analysis. A process needs to be developed that can utilize tools like Rational Rose that supports UML notation and ObjectTime that is based on ROOM notation.

At present DMM rules can be used to extract information from UML models only. Future DMMs need to be expanded to provide an integrated environment for extracting semantics from SART as well as UML. This is particularly useful in case of large systems with multiple CSCIs, where a some of the CSCIs are using UML notation and other CSCIs are using SART notation.

In summary the following areas have potential for future research in tool support:

- Mapping from SART to ROOM notation.

- An STU for UML specification models.

- Generating ROOM models for UML specification models.

- Extension of DMM for including other notations like SART.

- Extend this work to integrate MATRIXx specification tool, in the dynamic analysis framework presented in this dissertation. 


\subsubsection{Explore new dynamic specification metrics.}

Using the framework developed for SART, Ammar et. al. in [44], [55] and [56] explored the use of dynamic specification metrics for systems modeled using SART notation ( Section 6.3). Future work may be extend [44] towards similar analysis of UML models. That will require more research for establishing the dynamic complexity metrics for UML specifications. This is important because metrics based on static analysis such as "static complexity" may be misleading if it is merely reflecting the depth of the class hierarchy.

Dynamic complexity metrics would be more accurate because they consider only the functions being used in a given scenario. In this dissertation a framework for analyzing dynamic behavior of UML was developed. Dynamic analysis based on this framework can be used for generating performance and risk assessment metrics. Future research may focus on developing and refining such metrics.

\subsubsection{Methods for improving the design process of operational profiles}

Software reliability engineering (SRE) is used for developing reliable critical software components. An important task in SRE is designing the operational profiles. Operational profiles are built according to the user profile and the analyst's understanding of the system's dynamic behavior.

John Musa suggested that usage distribution can help in focusing on scenarios critical to the system's operation. He considered an example system of a phone exchange. For designing such a system, he identified three sets of users: an operator, a manager and a repair person. These users were assigned a value based on the expected time they would spend working on the exchange. The operator had $75 \%$ of usage, the manager had $12 \%$ of usage, and the repair-person had $13 \%$ of the usage. This distribution was utilized as a yard stick to design scenarios, critical to the system. 
Musa's approach described above, is based on static analysis of the system. Dynamic analysis can be used to confirm the results of Musa's study. For example, the scenarios with $75 \%$ of operator usage can actually be executed to determine the level of criticality. This will give a more realistic picture of the system usage.

In addition to the analysis just described, the framework proposed in this dissertation, can also be used for identifying high risk scenarios using input domain partitioning (in [45] by Cukic, Ammar, and Lateef). Dynamic models presented in Chapter 6 were utilized by Cukic for his work related to high risk scenarios.

The technique of dynamic analysis of usage distribution, as well as the technique for identifying high risk scenarios can help in the SRE process for improving the operational profiles. More research is needed to develop a methodology for incorporating these techniques within the SRE process. 


\section{Chapter 8 REFERENCES}

[1] E. Wang, Betty Cheng, et. al., "Formalizing and Integrating the Dynamic Model within OMT”, Proc. of IEEE International Conference on Software Engineering, pp45-55, May 1997.

[2] Technical report "Software IV\&V plan for Tactical Systems and Computer software", Intermetrics Inc. 1990.

[3] Jaffe, M.S.; Leveson, N.G., “Completeness, Robustness, And Safety In Real-time Software Requirements Specification”, 11th International Conference on Software Engineering, pp 302 - 311. Many 1989.

[4] Leveson, N.G.; Turner, C.S., "An investigation of the Therac-25 accidents", IEEE Computer, pp 18 - 4, Vol. 26, Issue: 7, July 1993

[5] Maier M.W., "Integrated Modeling: A unified Approach to system Engineering", The Journal of systems and software: Vol 32, pp101-119, 1996

[6] Fraser, M.D. \& Kumar, K. "Informal to formal requirement specification languages: Bridging the gap", IEEE transactions on Software Engineering, pp 454-, Vol. 17, No. 5, May 1991.

[7] Amoroso E. G., "Creating formal specifications from informal requirement documents", ACM SIGSOFT, Software Engineering Notes, Vol 20 no 1, pp 67-70, Jan 1995.

[8] Cooke D., et al., "Languages for the specification of the software", The Journal of systems and software: Vol 32, pp269-308, 1996.

[9] N. Dershowitz, "Program abstraction and instantiation", ACM Trans. Program. Languages 
and System., pp 446-477, Vol. 7, No. 3, October 1985.

[10] J.L. Peterson, "Petri Net Theory and the Modeling of Systems", Prentice Hall Inc., 1980.

[11] L. Cherkasova, V. Kotov, T. Rokicki: On Net Modeling of Industrial Size Concurrent Systems. Proceedings of the 14th International Petri Net Conference, Chicago 1993, Vol. 691, pp. 552-561.

[12] G. Booch, "Quality Software and the Unified Modeling Language”, a white paper, Rational software corporation, 1997.

[13] L. Baresi, M. Pezze, "Introducing Formal Specification Methods in Industrial Practice", International Conference on Software Engineering, 1997, pp56-66.

[14] Kung C.H., "Conceptual modeling in the context of software development", IEEE transactions on Software Engineering, pp 1176-1187, Vol. 15, No. 10, 1989

[15] Hooker s., Lockyer M.A., Fencott P.C., "CASE Support for Methods Integration: Implementation of translation from a structured to a formal notation", Proceedings of the Methods Integration Workshop, Leeds, 25-26 March 1996, Springer-Verlog.

[16] Andrews D., "Specification aspects of VDM", Information and software technology, vol 30, no. 3, pp164-176, April 1988.

[17] Wieringa R. J., "Combining Static and Dynamic Modeling Methods: A comparison of Four Methods", The Computer Journal, Vol 38, No. 1, 1995.

[18] Miriyala K., Harandi M.T., “Automatic Derivation of formal software specifications from informal descriptions", IEEE transactions on Software Engineering, pp 1126-1142, Vol. 17, No. 10, October 1991.

[19] Pezze M., Elmstrom R., Lintulampi R., "Giving Semantics to SA/RT by means of High- 
Level timed petri nets", The international journal of time critical computing systems, Vol. 5, no 2/3, May 1993

[20] "Automatic translation of SA/RT to high level time Petri nets", ESPIRIT report, IPTESPDM-17-V2.3. 1994

[21] B. Mikolajczak and J. Rumbut: A Systematic Method of Object-Oriented Software Design using Colored Petri Nets, Naval Underwater Warfare center; 14th International Conference on Applications and Theory of Petri Nets, Chicago, June 1993, pp. 21-25.

[22] Jens B. Jorgensen and Kjeld H. Mortensen, "Modeling and Analysis of Distributed Program Execution in BETA Using Colored Petrinets", Proc. of the 17th International Conference on Application and Theory of Petri Nets, Lecture Notes in Computer Science 1091, Osaka, Japan, June 1996. 249-268

[23] Kjeld H. Mortensen and Valerio Pinci, "Modeling the work flow of a Nuclear Waste Management Program", Proc. of the 15th International Conference on Application and Theory of Petrinets, Lecture Notes in Computer Science 815, Zaragoza, Spain, June 1994. 1994 Pages: $376-395$

[24] Rasmussen, Jens Linneberg; Singh, Mejar, "Designing a Security System by Means of Colored Petrinets: Application and Theory of Petrinets", Proc. 17th International Conference in Application and Theory of Petri Nets (ICATPN'96), Osaka, Japan --Springer-Verlag Jun, 1996 Pages: 400-419.

[25] T. Bolognesi and E. Brinksma, "Introduction to the ISO specification language LOTOS", in The Formal Description Technique LOTOS (P. H. J. V. Eijk, C. A. Vissers, and M. Diaz, eds.), pp. 23--73, North-Holland, 1989.

[26] Krishnan P., "Formal methods and design extraction: a pilot study" Information and 
software technology, vol 36(11), pp 675-681, 1994.

[27] H. W. Nissen, et al, "Managing Multiple Requirements Perspectives with Metamodels", IEEE Software, pp 37-48, March 1996.

[28] Artsy Y.S., "Meta-modeling the OO Methods, Tools, and Interoperability Facilities",. Metamodeling in OO, OOPSLA'95 Workshop October 15, 1995.

[29] Odell Jim, "Meta-Modeling", Metamodeling in OO, OOPSLA'95 Workshop October 15, 1995.

[30] Bucci G., Vicario E., "Compositional Validation of Time-Critical Systems using Communicating Time Petri Nets", IEEE transactions on software engineering, vol 21 no 12, pp 969-992, December 1995.

[31] Felder M., Mandrioli D., Morzenti A., "Proving properties of realtime systems through logical specifications and petri net models", IEEE transactions on software engineering, vol 20 no 2, pp 127-141, Feb 1994.

[32] Murata T., Notomi M., "Hierarchical Reachability Graph of Bounded Petri Nets for Concurrent-Software Analysis", IEEE transactions on Software Engineering, pp 325-336, Vol. 20, No. 5, May 1994.

[33] Sakthivel S., Moily J. P., “Analytical verification of information system requirements using Petrinets", Information and software technology, vol 35 no. 2, pp 89-100, February 1993.

[34] Buy U., Sloan R. H., "Analysis of Realtime Programs with simple time petri nets", ACM software engineering notes, special issue on ISSTA, pp 228-239, 1994.

[35] Ghezzi et al., "High-level timed petri nets as kernel for executable specifications", The 
international journal of time critical computing systems, Vol. 5, no 2/3, May 1993.

[36] Berthomieu et al., "Modeling and verification of time dependent systems using time petri nets", IEEE transactions on Software Engineering, pp 259-273, Vol. 17, March 1991

[37] Jensen K., "Coloured Petri nets: basic concepts, analysis methods and practical use", Springer-Verlag, Berlin; New York April 1992.

[38] Hatley Derek J, Pirbhai Imtiaz A, "Strategies for realtime system specification", Dorset House Pub, New York, NY1987.

[39] Fowler M., et. al. "UML Distilled: Applying the Standard Object Modeling Language", Addison-Wesley, 1997.

[40] K. Lateef, H. Ammar, "Identifying the Elements of Dynamic Metamodels from the Requirement Specifications of Parallel and Distributed Software Systems", IEEE International Conference on computers and systems, Lahore University of Management Sciences, Pakistan, November 1998.

[41] K. Lateef, H.H. Ammar, V. Mogulothu, T. Nikzadeh, "A Methodology for Verification and Analysis of Parallel and Distributed Systems Requirement Specifications", in Proceedings of the 2nd IFIP International Workshop on Software Engineering for Parallel and Distributed Systems (PDSE-97), IEEE Computer Society, May 1997.

[42] L. Baresi, M. Pezze, "Introducing Formal Specification Methods in Industrial Practice", International Conference on Software Engineering, 1997, pp56-66.

[43] V. Mogulothu, H.H. Ammar, K. Lateef, T. Nikzadeh, "Performability Analysis of the Commanding Component of NASA's Earth Observing System”, Proceedings of the 10th International Conference on Parallel and Distributed Computing systems, IEEE Computer 
Society, October 1997.

[44] Hany Ammar Khalid Lateef Vinay Mogulothu Tooraj Nikzadeh, "A Methodology for Risk Assessment and Performability Analysis of Large Scale Software Systems", International conference on computer systems, Cairo, Egypt, 1997.

[45] B. Cukic, H.H.Ammar, K. Lateef, "Identifying High-Risk Scenarios of complex Systems Using Input Domain Partitioning”, International Symposium on Software Reliability, Paderborn, Germany, 1998.

[46] Harel D., Gery E., "Executable Object Modeling with Statecharts", IEEE Computer, Vol. 30, No. 7., July 1997.

[47] DSTC, IBM, Oracle, Platinum Technology and Unisys, Joint Initial Submission to the SMIF RFP. OMG document ad/98-07-01, 1998.

[48] Odell Jim, "Meta-Modeling”, Metamodeling in OO, OOPSLA'95 Workshop October 15, 1995.

[49] Alan Davis, "A comparison of techniques for the specification of external system behavior", pp 237-254, Software Requirements Engineering Journal, IEEE computer society, 1997.

[50] Alford, M. “A requirement engineering methodology for realtime processing requirements", pp 60-69, IEEE transactions on Software Engineering, 3, 1 January 1977.

[51] Alan Davis, "Requirements language processing for the effective testing of realtime software”, pp 61-66, ACM software engineering Notes 3, 5 November 1978.

[52] Douglas B., "Realtime UML”, Addison-Wesley Object Technology Series, 1997.

[53] Functional and Performance Requirements Specification for the Earth Observing System 
Data and Information System (EOSDIS) Core System. Revision A and CH-01.

[54] Flight Operations Segment (FOS) Requirements Specification for the ECS Project, Volume 1: General Requirements, November 1994. By Hughes Applied Information Systems.

[55] H. Ammar, T. Nikzadeh, and J. B. Dugan "Risk Assessment of Functional Specification of Software Systems Using Colored Petri Nets," Proceedings of the International Symposium On Software Reliability Engineering (ISSRE'97), IEEE Computer Society, November 1997.

[56] H. Ammar, T. Nikzadeh, and J. B. Dugan "A Methodology for Risk Assessment of Functional Specifications using Colored Petri nets," in proceedings of the Fourth International Software Metrics Symposium (Metrics'97), IEEE Computer Society, November 1997. 